\title{
The Dispersion of the Gauss-Markov Source
}

\author{
Peida Tian, Victoria Kostina
}

\begin{abstract}
The Gauss-Markov source produces $U_{i}=a U_{i-1}+$ $Z_{i}$ for $i \geq 1$, where $U_{0}=0,|a|<1$ and $Z_{i} \sim \mathcal{N}\left(0, \sigma^{2}\right)$ are i.i.d. Gaussian random variables. We consider lossy compression of a block of $n$ samples of the Gauss-Markov source under squared error distortion. We obtain the Gaussian approximation for the Gauss-Markov source with excess-distortion criterion for any distortion $d>0$, and we show that the dispersion has a reverse waterfilling representation. This is the first finite blocklength result for lossy compression of sources with memory. We prove that the finite blocklength rate-distortion function $R(n, d, \epsilon)$ approaches the rate-distortion function $\mathbb{R}(d)$ as $R(n, d, \epsilon)=$ $\mathbb{R}(d)+\sqrt{\frac{V(d)}{n}} Q^{-1}(\epsilon)+o\left(\frac{1}{\sqrt{n}}\right)$, where $V(d)$ is the dispersion, $\epsilon \in(0,1)$ is the excess-distortion probability, and $Q^{-1}$ is the inverse of the $Q$-function. We give a reverse waterfilling integral representation for the dispersion $V(d)$, which parallels that of the rate-distortion functions for Gaussian processes. Remarkably, for all $0<d \leq \frac{\sigma^{2}}{(1+|a|)^{2}}, R(n, d, \epsilon)$ of the Gauss-Markov source coincides with that of $Z_{i}$, the i.i.d. Gaussian noise driving the process (whose dispersion is $1 / 2$ ), up to the second-order term. Among novel technical tools developed in this paper is a sharp approximation of the eigenvalues of the covariance matrix of $n$ samples of the Gauss-Markov source, and a construction of a typical set using the maximum likelihood estimate of the parameter $a$ based on $n$ observations.
\end{abstract}

Index Terms-Lossy source coding, Gauss-Markov source, dispersion, finite blocklength regime, rate-distortion theory, sources with memory, achievability, converse, autoregressive processes.

\section{INTRODUCTION}

In rate-distortion theory [1] [2], a source, modeled as a discrete stochastic process $\left\{U_{i}\right\}_{i=0}^{\infty}$, produces a random vector $\mathbf{U} \triangleq\left(U_{1}, \ldots, U_{n}\right)$ and the goal is to represent $\mathbf{U}$ by minimum number of reproduction vectors $\mathbf{V}$ such that the distortion is no greater than a given threshold $d$. For any such set of reproduction vectors, the associated rate is defined as the ratio between the logarithm of the number of vectors and $n$. The rate quantifies the minimum number of bits per symbol needed to describe the source with distortion $d$.

Numerous studies have been pursued since the seminal paper [2], where Shannon first proved the rate-distortion theorem for discrete stationary memoryless sources (DMS) and then for stationary ergodic sources. Shannon's rate-distortion theorem shows that the minimum rate needed to describe a DMS within distortion $d$ is given by the rate-distortion function (RDF) $\mathbb{R}(d)$, which is computed as a solution to a (single-letter) minimal mutual information convex optimization problem. Goblick [3] proved a coding theorem for general

P. Tian and V. Kostina are with the Department of Electrical Engineering, California Institute of Technology. (e-mail: \{ptian, vkostina\} @caltech.edu) This research was supported in part by the National Science Foundation (NSF) under Grant CCF-1566567. A shorter version of this paper was accepted for publication by the IEEE International Symposium on Information Theory, Vail, CO, USA, June 2018. ergodic sources showing that the RDF is equal to the limit of $n$ letter minimal mutual informations. That limit has exponential computational complexity in general. Computable expressions for the RDF of sources with memory are known only in the following special cases. Gray [4] showed a closed-form expression for the RDF for a binary symmetric Markov source with bit error rate distortion in a low distortion regime. For higher distortions, Jalali and Weissman [5] recently showed upper and lower bounds allowing one to compute the ratedistortion function in this case with desired accuracy. Gray [6] showed a lower bound to the rate-distortion function of finitestate finite-alphabet Markov sources with a balanced distortion measure, and the lower bound becomes tight when $d \in\left(0, d_{c}\right]$ for critical distortion $d_{c}$. For the mean squared error distortion measure (MSE), Davisson [7], and also Kolmogorov [8], derived the rate-distortion function for stationary Gaussian processes by applying a unitary transformation to the process to decorrelate it and applying reverse waterfilling to the decorrelated Gaussians [9]. Berger [10] and Gray [4], in separate contributions in the late 60's and early 70's, derived the MSE rate-distortion function for Gaussian autoregressive sources.

All of the above mentioned work [1]-[7], [9], [10] apply to the operational regime where the coding length $n$ grows without bound. Asymptotic coding theorems are important since they set a clear boundary between the achievable and the impossible. However, practical compression schemes are of finite blocklength. A natural, but challenging, question to ask is: for a given coding blocklength $n$, what is the minimum rate to compress the source with distortion at most $d$ ? Answering this question exactly is hard. An easier question is that of second-order analysis, which studies the dominating term in the gap between RDF and the finite blocklength minimum rate.

In rate-distortion theorems, the landscape of second-order analyses consists of two criteria: average distortion and excess distortion. The average distortion constraint posits that the average distortion should be at most $d$, while excess distortion constraint requires that the probability of distortion exceeding $d$ be at most $\epsilon$. For average distortion criterion, Zhang, Yang and Wei [11] proved that for i.i.d. finite alphabet sources, the leading term in the gap $R(n, d)-\mathbb{R}(d)$ is $\frac{\log n}{2 n}$, where $R(n, d)$ denotes the minimum rate compatible with average distortion $d$ at coding length $n$. Later, Yang and Zhang [12] extended the achievability result of [11] to abstract sources.

For lossy compression of i.i.d. sources under excess distortion criterion, the minimum achievable finite blocklength rate admits the following expansion [13], [14], known as the

\footnotetext{
${ }^{1}$ This statement is translated from [11], where the equivalent result was stated in terms of distortion-rate function.
} 
Gaussian approximation:

$$
R(n, d, \epsilon)=\mathbb{R}(d)+\sqrt{\frac{V(d)}{n}} Q^{-1}(\epsilon)+O\left(\frac{\log n}{n}\right),
$$

where $V(d)$ is referred to as the source dispersion and $Q^{-1}(\cdot)$ denotes the inverse of $Q$-function. Extensions of the result in 11 to joint source-channel coding [15] [16] and multiterminal source coding [17] [18] have also been studied.

The dispersion of lossy compression of sources with memory is unknown. In the context of variable-length lossy compression with guaranteed distortion, Kontoyiannis [19, Th. 6, Th. 8] established a connection between the number of bits needed to represent $n$ given samples produced by an arbitrary source, and the logarithm of the reciprocal of distortion $d$-ball probability. Unfortunately computation of that probability has exponential in $n$ complexity. In contrast, the dispersions of lossless compression of sources with memory and of channel coding over channels with memory are known in some cases. The second-order expansion of the minimum encoded length in the lossless compression of Markov sources is computed in [20], [21]. Polyanskiy et al. presented a waterfilling representation for the channel dispersion of the Gaussian channel with intersymbol interference in [22, Th. 5], and found the dispersion of the Gilbert-Elliott channel in [23, Th. 4].

In this paper, we derive an expansion of type (1) on $R(n, d, \epsilon)$ for the Gauss-Markov source, one of the simple models for sources with memory. The dispersion $V(d)$ for the GaussMarkov source is shown to be the limiting variance of $d$ tilted information, and has a reverse waterfilling representation. The dispersion $V(d)$ for low distortions is shown to be the same as that of the i.i.d. Gaussian noise driving the process, which estends the corresponding result of Gray [4, Eq. (24)] to the nonasymptotic regime. These main results are presented in Section III Our converse proof generalizes to Gaussian autoregressive processes [4], but the achievability proof does not. In proving the converse and achievability, we develop several new tools including a nonasymptotic refinement of Gray's result [4. Eq. (19)] on the eigenvalue distribution of the covariance matrix of the Gauss-Markov source. This refinement relies on a sharp bound on the differences of eigenvalues of two sequences of tridiagonal matrices, proved using Cauchy interlacing theorem and Gershgorin circle theorem from matrix analysis. In proving achievability, we derive a maximum likelihood estimator of the parameter $a$ of the Gauss-Markov source and bound the estimation error using a concentration inequality on multilinear polynomials [24]. Our key tool in achievability proof is the construction of a typical set based on the maximum likelihood estimator. Proofs of converse and achievability are presented in Section III and Section IV respectively. Finally, we conclude in Section $\mathrm{V}$ with brief discussions on some open problems. Fig. 4 in Appendix A-A presents a roadmap containing the relations of all theorems, corollaries and lemmas in this paper.

Notations: Throughout, lowercase (uppercase) boldface letters denote vectors (random vectors) of length $n$. We omit the dimension where there is no ambiguity, i.e. $\mathbf{u} \equiv \mathbf{u}^{n} \equiv$ $\left(u_{1}, \ldots, u_{n}\right)$ and $\mathbf{U} \equiv \mathbf{U}^{n} \equiv\left(U_{1}, \ldots, U_{n}\right)$. For a random variable $X$, we use $\mathbb{E}[X]$ and $\operatorname{Var}[X]$ to denote its mean and variance, respectively. All logarithms are base $e$. For any positive integer $m$, we denote by $[m]$ the set of intergers $\{1,2, \ldots, m\}$. We use the standard $O(\cdot), o(\cdot)$ and $\Theta(\cdot)$ notations to characterize functions according to their asymptotic growth rates. Namely, let $f(n)$ and $g(n)$ be two functions on $n$, then $f(n)=O(g(n))$ if and only if there exists positive real number $M$ and $n_{0} \in \mathbb{N}$ such that $|f(n)| \leq M|g(n)|$ for any $n \geq n_{0}$; $f(n)=o(g(n))$ if and only if $\lim _{n \rightarrow \infty} f(n) / g(n)=0$; $f(n)=\Theta(g(n))$ if and only there exist positive constants $c_{1}, c_{2}$ and $n_{0} \in \mathbb{N}$ such that $c_{1} g(n) \leq f(n) \leq c_{2} g(n)$ for any $n \geq n_{0}$. We write matrices using sans serif font, e.g. a matrix $A$, and we denote by $\mathbb{1}\{\cdot\}$ the indicator function.

\section{Problem Formulation And Main Results}

\section{A. Source model and definitions}

The Gauss-Markov source $\left\{U_{i}\right\}_{i=0}^{\infty}$ satisfies the following difference equation:

$$
U_{i}=a U_{i-1}+Z_{i}
$$

for $i \geq 1$, and $U_{0}=0$. Here, $a \in[0,1)$ is the gain ${ }^{2}$ and $Z_{i}$ 's are the innovations, independently and identically distributed (i.i.d.) Gaussian random variables, i.e., $Z_{i} \sim \mathcal{N}\left(0, \sigma^{2}\right)$. We adopt (2) as the simplest model capturing information sources with memory: gain $a$ determines how much memory (as well as the growth rate), and the innovation $Z_{i}$ represents new information being generated at each time step. This is the firstorder autoregressive process, whose rate-distortion function was studied in [4], [25], [26].

To formally set up the problem of lossy compression of the Gauss-Markov source, we proceed to introduce the following definitions. Let $\mathbf{u}, \mathbf{v} \in \mathbb{R}^{n}$. The distortion between $\mathbf{u}$ and $\mathbf{v}$, denoted by $d(\mathbf{u}, \mathbf{v})$, is the normalized squared Euclidean distance (mean squared error) between $\mathbf{u}$ and $\mathbf{v}$ :

$$
\mathrm{d}(\mathbf{u}, \mathbf{v}) \triangleq \frac{1}{n} \sum_{i=1}^{n}\left(u_{i}-v_{i}\right)^{2}=\frac{1}{n}\|\mathbf{u}-\mathbf{v}\|_{2}^{2} .
$$

An $(M, n)$ code consists of an encoding function $\mathrm{f}_{n}: \mathbb{R}^{n} \rightarrow$ $[M]$, and a decoding function $\mathrm{g}_{n}:[M] \rightarrow \mathbb{R}^{n}$. The rate associated with an $(M, n)$ code is defined as $R \triangleq \frac{\log M}{n}$.

Definition 1 (Excess distortion code). For $d>0$ and $\epsilon \in[0,1]$, an $(n, M, d, \epsilon)$-excess distortion code is an $(M, n)$ code such that

$$
\mathbb{P}\left[\mathrm{d}\left(\mathbf{U}, \mathrm{g}_{n}\left(\mathrm{f}_{n}(\mathbf{U})\right)\right) \geq d\right] \leq \epsilon .
$$

The non-asymptotic fundamental limits under the excess distortion criterion, that is, the operational quantities of interest, are defined as follows. Given $n \in \mathbb{N}$ and $d>0$, the minimum achievable code size and the minimum achievable rate at blocklength $n$ and distortion $d$ are defined as

$M^{\star}(n, d, \epsilon) \triangleq \inf \{M: \exists(n, M, d, \epsilon)$-excess distortion code $\}$

$$
R(n, d, \epsilon) \triangleq \frac{\log M^{\star}(n, d, \epsilon)}{n} .
$$

\footnotetext{
${ }^{2}$ Note that if $a \in(-1,0]$ in $[2]$, then $\left\{(-1)^{i} U_{i}\right\}_{i=0}^{\infty}$ is a Gauss-Markov source with nonnegative gain $-a$ and the same innovation variance. Thus restricting to $0 \leq a<1$ is without loss of generality.
} 
Given $n \in \mathbb{N}$ and $d>0$, the $n$-th order (informational) ratedistortion function ( $n$-th order RDF) is defined as

$$
\mathbb{R}_{\mathbf{U}}(n, d) \triangleq \frac{1}{n} \inf _{\substack{P \mathbf{V} \mid \mathbf{U}: \\ \mathbb{E}[\mathrm{d}(\mathbf{U}, \mathbf{V})] \leq d}} I(\mathbf{U} ; \mathbf{V}),
$$

where $I(\cdot ; \cdot)$ is the mutual information. We say that $\left(\mathbf{U}, \mathbf{V}^{*}\right)$ is a RDF-achieving pair if its joint distribution is $P_{\mathbf{U}} P_{\mathbf{V}^{*} \mid \mathbf{U}}$ where $P_{\mathbf{V}^{*} \mid \mathbf{U}}$ is a minimizer of the optimization problem (7). For any $\mathbf{u} \in \mathbb{R}^{n}$, the d-tilted information $\jmath_{\mathbf{U}}(\mathbf{u}, d)$ in $\mathbf{u}$, introduced by [13], is

$$
\jmath_{\mathbf{U}}(\mathbf{u}, d) \triangleq-\lambda^{\star \star} n d-\log \mathbb{E}\left[\exp \left(-\lambda^{\star \star} n \mathbf{d}\left(\mathbf{u}, \mathbf{V}^{\star}\right)\right)\right],
$$

where

$$
\lambda^{\star \star} \triangleq-\mathbb{R}_{\mathbf{U}}^{\prime}(n, d),
$$

and the derivative is with respect to $d$. The normalized expected value of d-tilted information yields the $n$-th order RDF:

$$
\mathbb{R}_{\mathbf{U}}(n, d)=\frac{1}{n} \mathbb{E}\left[\jmath_{\mathbf{U}}(\mathbf{U}, d)\right] .
$$

The rate-distortion function (RDF) is

$$
\mathbb{R}_{\mathbf{U}}(d) \triangleq \lim _{n \rightarrow \infty} \mathbb{R}_{\mathbf{U}}(n, d) .
$$

In terms of coding theorems, the equality between $R(d)$ (the minimum achievable source coding rate under average distortion criterion when the blocklength $n$ goes to infinity) and $\mathbb{R}_{\mathbf{U}}(d)$ (the informational rate-distortion function) has been established [2], [10, Th. 6.3.4], [4, Th. 2]. For the excess distortion criterion, Shannon's achievability in [2] and Kieffer's strong converse [27] for stationary ergodic sources together imply that for the Gauss-Markov source $\mathbf{U}$ in (2) , for any $d \in\left(0, d_{\max }\right)$ and $\epsilon \in(0,1)$,

$$
\lim _{n \rightarrow \infty} R(n, d, \epsilon)=\mathbb{R}_{\mathbf{U}}(d) .
$$

The rate-dispersion function $V(d)$, introduced in [13, Def. 7] and simply referred to as dispersion in this paper, is defined as

$$
V(d) \triangleq \lim _{\epsilon \rightarrow 0} \lim _{n \rightarrow \infty} n\left(\frac{R(n, d, \epsilon)-R(d)}{Q^{-1}(\epsilon)}\right)^{2} .
$$

Roughly speaking, the square root of the dispersion $V(d)$ is the ratio between the leading term in the gap $R(n, d, \epsilon)-$ $R(d)$ and $\frac{Q^{-1}(\epsilon)}{\sqrt{n}}$. The main result of this paper is a complete characterization of the dispersion $V(d)$ for the Gauss-Markov source in (2).

\section{B. Previous work}

The reverse waterfilling solution originated in the work of Kolmogorov [8] on RDF of Gaussian processes. Let $\left\{U_{i}\right\}_{i=-\infty}^{+\infty}$ be a stationary Gaussian random process. The $n$-th order RDF $\mathbb{R}_{\mathbf{U}}(n, d)$ for $\mathbf{U}=\left(U_{1}, \ldots, U_{n}\right)$ is given by the $n$-th order reverse waterfilling solution:

$$
\begin{aligned}
\mathbb{R}_{\mathbf{U}}(n, d) & =\frac{1}{n} \sum_{i=1}^{n} \max \left(0, \frac{1}{2} \log \frac{\sigma_{i}^{2}}{\theta_{n}}\right), \\
d & =\frac{1}{n} \sum_{i=1}^{n} \min \left(\theta_{n}, \sigma_{i}^{2}\right),
\end{aligned}
$$

where $\sigma_{i}^{2}, i \in[n]$ are the eigenvalues of the covariance matrix $\Sigma_{\mathbf{U}} \triangleq \mathbb{E}\left[\mathbf{U U}^{T}\right]$, and $\theta_{n}>0$ is the water level matched to $d$ at blocklength $n$ via (15). The RDF of the Gaussian random process is given by the limiting reverse waterfilling solution [10], [28], depicted in Fig. 1a]

$$
\begin{aligned}
\mathbb{R}_{\mathbf{U}}(d) & =\frac{1}{2 \pi} \int_{-\pi}^{\pi} \max \left[0, \frac{1}{2} \log \frac{S(w)}{\theta}\right] d w, \\
d & =\frac{1}{2 \pi} \int_{-\pi}^{\pi} \min (\theta, S(w)) d w,
\end{aligned}
$$

where $S(w)$ is the power spectrum of the Gaussian random process, and $\theta>0$ is the water level matched to $d$ via (17). The power spectrum $S(w)$ is defined as

$$
S(w) \triangleq \sum_{i \in \mathbb{N}} \mathbb{E}\left[U_{n} U_{n+i}\right] e^{-\mathrm{j} i w}, \quad w \in[-\pi, \pi] .
$$

Gray [4] and Berger [10, Th. 6.3.2] studied the RDF for Gaussian autoregressive processes, which evolve as [4]

$$
U_{i}=\sum_{j=1}^{i} a_{j} U_{i-j}+Z_{i}, \quad i \geq 1,
$$

where $Z_{i} \sim \mathcal{N}\left(0, \sigma^{2}\right), i \geq 1$ are i.i.d. Gaussian random variables, the real constants $a_{j}$ 's are assumed to satisfy [4 Eq. (10)]

$$
\sum_{j=0}^{\infty}\left|a_{j}\right|<\infty
$$

and $U_{i}=0$ for $i \leq 0$. The Gauss-Markov source in (2) is a special case of (19) with $a_{1}=a$ and $a_{i}=0$ for $i \geq 2$. The following parametric representation of $\operatorname{RDF} \mathbb{R}_{\mathbf{U}}(d)$ for Gauss-Markov source $\mathbf{U}$ in (2) is immediate from Berger's result in [10, Th. 6.3.2]: ${ }^{3}$

$$
\begin{aligned}
\mathbb{R}_{\mathbf{U}}(d) & =\frac{1}{2 \pi} \int_{-\pi}^{\pi} \max \left[0, \frac{1}{2} \log \frac{\sigma^{2}}{\theta g(w)}\right] d w, \\
d & =\frac{1}{2 \pi} \int_{-\pi}^{\pi} \min \left[\theta, \frac{\sigma^{2}}{g(w)}\right] d w,
\end{aligned}
$$

where the power spectrum of the Gauss-Markov source (2) is given by

$$
S(w)=\frac{\sigma^{2}}{g(w)},
$$

and the function $g$ is defined as

$$
g(w) \triangleq 1+a^{2}-2 a \cos (w), \quad \forall w \in[-\pi, \pi] .
$$

Gray [4] also showed the following relation between the RDF $\mathbb{R}_{\mathbf{U}}(d)$ of a Gaussian autoregressive process $\mathbf{U}$ and the RDF $\mathbb{R}_{\mathbf{Z}}(d)$ of the i.i.d. innovation process $\left\{Z_{i}\right\}_{i=1}^{\infty}$ :

$$
\left\{\begin{array}{l}
\mathbb{R}_{\mathbf{U}}(d)=\mathbb{R}_{\mathbf{Z}}(d), \quad 0<d \leq d_{c}, \\
\mathbb{R}_{\mathbf{U}}(d)>\mathbb{R}_{\mathbf{Z}}(d), \quad d_{c}<d \leq d_{\max }
\end{array}\right.
$$

${ }^{3}$ In 2, the source is stationary since $|a|<1$. In nonstationary case $(|a| \geq 1)$, the rate-distortion function is given by the right-hand side of (21) plus $\log |a|$ [4], [25], [26]; the critical distortion is given by [26; and $d_{\max }=+\infty$ (stationary variance does not exist). 
where $d_{c}$ is referred to as the critical distortion, defined as

$$
d_{c} \triangleq \min _{w \in[-\pi, \pi]} S(w)
$$

and $d_{\max }$ is the maximum distortion achievable in (22), that is,

$$
d_{\max } \triangleq \frac{1}{2 \pi} \int_{-\pi}^{\pi} \frac{\sigma^{2}}{g(w)} d w
$$

and

$$
\mathbb{R}_{\mathbf{Z}}(d)=\max \left(0, \frac{1}{2} \log \frac{\sigma^{2}}{d}\right), \quad \forall d>0,
$$

is the RDF for i.i.d. Gaussian source derived by Shannon [2]. Note that $d_{\max }$ equals the stationary variance of the source (2), i.e.,

$$
d_{\max }=\lim _{n \rightarrow \infty} \operatorname{Var}\left[U_{n}\right] .
$$

which is verified in Appendix A-D Equality in 25) is a deep result stating that in a range of low distortions, the asymptotic rate-distortion tradeoff of a Gaussian autoregressive process and its driving innovation process are the same. See Fig. $1 \mathrm{~b}$ for an illustration of 25 for a Gauss-Markov source. In that case, the critical distortion is given by

$$
d_{c}=\frac{\sigma^{2}}{(1+a)^{2}},
$$

and maximum distortion is given by

$$
d_{\max }=\frac{\sigma^{2}}{1-a^{2}}
$$

The main result of this paper is the Gaussian approximation expansion of the limit in (12), presented next.

\section{Main Results}

Our main contribution is the Gaussian approximation of $R(n, d, \epsilon)$, detailed in the following theorem.

Theorem 1 (Gaussian approximation for Gauss-Markov source). Fix any $\epsilon \in(0,1)$ and $d \in\left(0, d_{\max }\right)$, where $d_{\max }$ is defined in (31). The minimum achievable source coding rate for the Gauss-Markov source $\mathrm{U}$ in (2) satisfies

$$
R(n, d, \epsilon)=\mathbb{R}_{\mathbf{U}}(d)+\sqrt{\frac{V(d)}{n}} Q^{-1}(\epsilon)+o\left(\frac{1}{\sqrt{n}}\right),
$$

where $\mathbb{R}_{\mathbf{U}}(d)$ is the rate-distortion function of the GaussMarkov source, given by (21), and the dispersion $V(d)$ is given by

$$
V(d)=\frac{1}{4 \pi} \int_{-\pi}^{\pi} \min \left[1,\left(\frac{\sigma^{2}}{\theta g(w)}\right)^{2}\right] d w,
$$

where $\theta>0$ is the water level matched to the distortion $d$ via (22), and the function $g$ is defined in (24).

The proof of Theorem 1 consists of converse and achievability, presented in Sections III and IV below.

Observe the pleasing parallel between the new reverse waterfilling solution for the dispersion in (33) and the classical reverse waterfilling representation of the rate-distortion function in (21). Furthermore, just like their rate-distortion functions, the dispersions of $\mathbf{U}$ and its innovation process $\mathbf{Z}$ are comparable:

Corollary 1. Let $V_{\mathbf{U}}(d)$ and $V_{\mathbf{Z}}(d)$ be the dispersions of the Gauss-Markov source (2) and the memoryless Gaussian source $\left\{Z_{i}\right\}_{i=1}^{\infty}$, respectively, i.e., $V_{\mathbf{U}}(d)$ is given by (33) and $V_{\mathbf{Z}}(d)=$ $1 / 2, \forall 0<d<\sigma^{2}$, then

$$
\begin{cases}V_{\mathbf{U}}(d)=V_{\mathbf{Z}}(d), & 0<d \leq d_{c} \\ V_{\mathbf{U}}(d)<V_{\mathbf{Z}}(d), & d_{c}<d<\sigma^{2}\end{cases}
$$

Proof. Corollary 1 is immediate by noting that the minimum in (33) equals 1 for $d \in\left(0, d_{c}\right]$ and is less than 1 when $d>d_{c}$.

Corollary 1 parallels Gray's result [25] [4] for the ratedistortion functions of $\mathbf{U}$ and $\mathbf{Z}$, and they together imply that for $d \in\left(0, d_{c}\right]$, the fundamental limites of lossy compression of the Gauss-Markov source and the i.i.d. Gaussian source $\left\{Z_{i}\right\}_{i=1}^{\infty}$ are the same, up to the second-order term. For $d \in\left(d_{c}, \sigma^{2}\right)$, the Gauss-Markov source is harder to compress in the limit of $n$ going to infinity since $\mathbb{R}_{\mathbf{U}}(d)>\mathbb{R}_{\mathbf{Z}}(d)$, but the Gauss-Markov source approaches its asymptotic fundamental limit faster since $V_{\mathbf{U}}(d)<V_{\mathbf{Z}}(d)$.

The dispersions $V(d)$ for $a=0$ and $a=0.5$ are plotted in Fig. 2. where the dotted line (for $a=0, \sigma^{2}=1$ ) recovers the dispersion result [13], [14] for i.i.d. Gaussian source $\left\{Z_{i}\right\}_{i=1}^{\infty}$, as expected. The solid line ( for $a=0.5, \sigma^{2}=1$ ) coincides with the dotted line in the region $d \in\left(0, d_{c}\right]$, which means that the Gauss-Markov source has the same dispersion as its innovation process in the region of low $d$ 's. For $d \in\left(d_{c}, \sigma^{2}\right)$, the dispersion of the Gauss-Markov source is smaller than that of its innovation process and decreases with $d$. Using the residue theorem from complex analysis (see Appendix A-C for details), we also derive the coordinates of the two corner points $P_{1}$ and $P_{2}$ on the solid line:

$$
P_{1}=\left(d_{c}, 1 / 2\right), \quad P_{2}=\left(d_{\max }, \frac{\left(1+a^{2}\right)(1-a)}{2(1+a)^{3}}\right) .
$$

By analogy with the informational RDF, defined in (11), we define the informational dispersion as the limiting variance of d-tilted information (defined in (8)):

$$
\mathbb{V}(d) \triangleq \lim _{n \rightarrow \infty} \frac{1}{n} \operatorname{Var}[\jmath \mathbf{U}(\mathbf{U}, d)] .
$$

Theorem 2 below establishes equivalence between the informational dispersion in (36) and the operational dispersion in (13), which we computed in Theorem 1 by reverse waterfilling.

Theorem 2. For the Gauss-Markov source $\mathbf{U}$ in (2) and for any $d \in\left(0, d_{\max }\right)$,

$$
\mathbb{V}(d)=V(d)
$$

where $V(d)$ is given in (33).

Proof. Appendix B-D. 


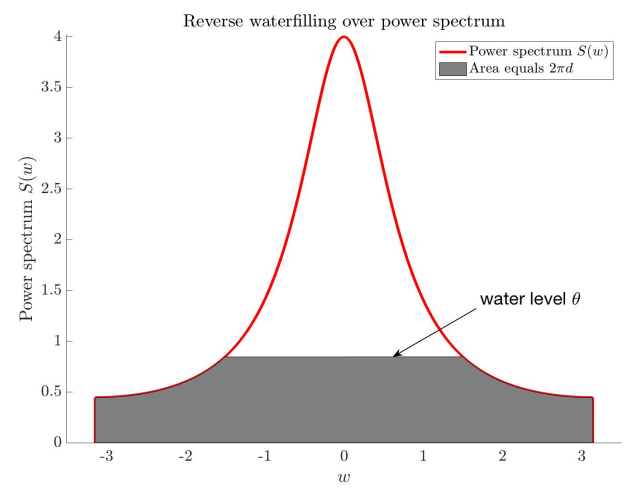

(a) Reverse waterfilling (17) over the power spectrum: the water level $\theta$ is chosen so that the shaded area equals $2 \pi d$.

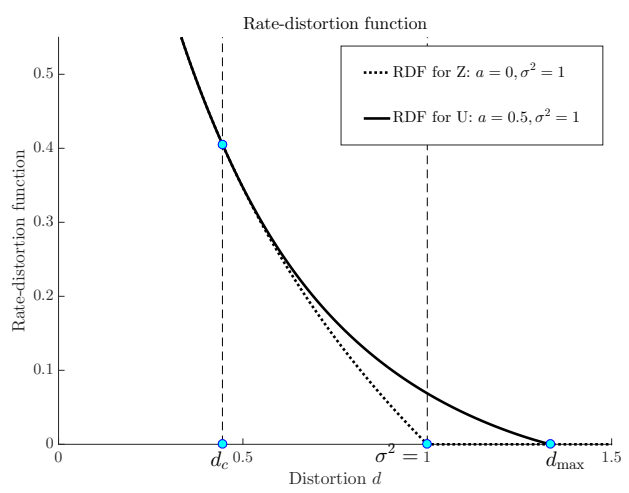

(b) The rate-distortion functions for the Gauss-Markov source $\left\{U_{i}\right\}$ with $a=0.5$ and $\sigma^{2}=1$, and for the innovation process $\left\{Z_{i}\right\}_{i=1}^{\infty}, Z_{i} \sim \mathcal{N}(0,1)$ driving that source.

Fig. 1: Reverse waterfilling solution and rate-distortion functions.

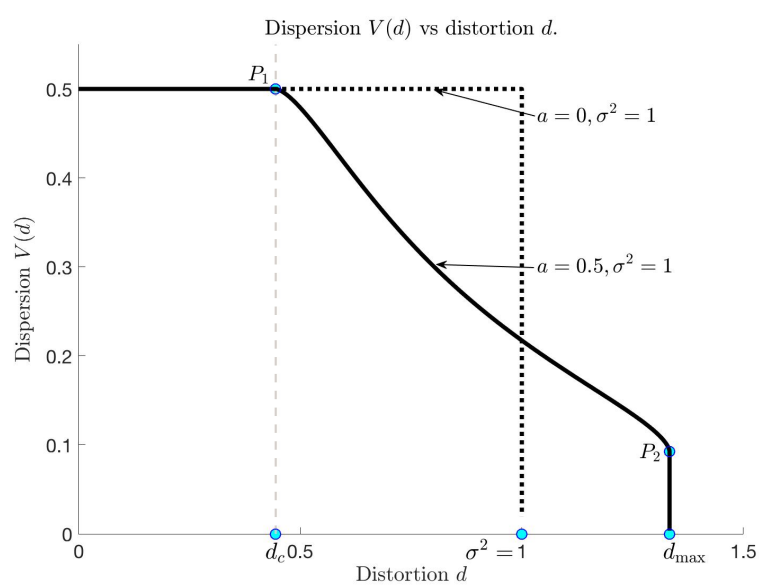

Fig. 2: Dispersion $V(d)$ versus distortion $d$ : The Gauss-Markov source with $a=0$ and $\sigma^{2}=1$ (dotted line) degenerates to the i.i.d. Gaussian source, i.e., the innovation process $\left\{Z_{i}\right\}_{i=1}^{\infty}$. The dispersion of the Gauss-Markov source with $a=0.5$ and $\sigma^{2}=1$ is given by the solid line. Two corner points on the solid line are labeled as $P_{1}$ (corresponding to $d_{c}$ ) and $P_{2}$ (corresponding to $d_{\max }$ ). The vertical segment between $\left(d_{\text {max }}, 0\right)$ and $P_{2}$ corresponds to the case when the water level $\theta$ is already above $\theta_{\max }$ (the water level matched to $d_{\max }$ ), and the dispersion $V(d)$ continues decreasing when distortion $d$ remains as $d_{\max }$, as seen from (22) and (33).

Note that (10) and 21) imply that the limit of normalized expectations of $n$-dimensional d-tilted information is given by the reverse waterfilling solution. That is,

$$
\lim _{n \rightarrow \infty} \frac{1}{n} \mathbb{E}\left[\jmath_{\mathbf{U}}(\mathbf{U}, d)\right]=\mathbb{R}_{\mathbf{U}}(d),
$$

which is due to Gray [4]. Theorem 2 extends the result into second moment. Furthermore, Theorem 2 provides a natural extension to the known result concerning lossy compression of i.i.d. sources $\left\{X_{i}\right\}[13]$, which states that the mean $\mathbb{E}\left[\jmath_{X_{1}}\left(X_{1}, d\right)\right]$ and the variance $\operatorname{Var}\left[\jmath_{X_{1}}\left(X_{1}, d\right)\right]$ of single- letter d-tilted information $\jmath_{X_{1}}\left(X_{1}, d\right)$ are equal to the RDF and the dispersion, respectively.

\section{Preliminary analyses}

1) Decorrelation: For each $n$, the random vector $\mathbf{U}=$ $\left(U_{1}, \ldots, U_{n}\right)$ generated by the model (2) follows the multivariate Gaussian distribution $\mathcal{N}\left(\mathbf{0}, \Sigma_{\mathbf{U}}\right)$, where $\Sigma_{\mathbf{U}}=\sigma^{2}\left(\mathrm{~A}^{T} \mathrm{~A}\right)^{-1}$ is its covariance matrix, and $\mathrm{A}$ is an $n \times n$ lower triangular matrix, defined as follows:

$$
\mathrm{A}=\left[\begin{array}{ccccc}
1 & 0 & 0 & \ldots & 0 \\
-a & 1 & 0 & \ldots & 0 \\
0 & -a & 1 & \ldots & 0 \\
\vdots & \ddots & \ddots & \ddots & \vdots \\
0 & \ldots & 0 & -a & 1
\end{array}\right]
$$

Note that $A$ is invertible since $\operatorname{det} A=1$. Since the difference equation 2 can be rewritten as $\mathbf{Z}=\mathbf{A} \mathbf{U}$, and $\mathbf{Z} \sim \mathcal{N}\left(\mathbf{0}, \sigma^{2} \mathbf{I}\right)$, the following simple calculation yields the covariance matrix of $\mathbf{U}$ :

$$
\Sigma_{\mathbf{U}}=\mathbb{E}\left[\mathbf{U U}^{T}\right]=\mathbb{E}\left[\mathrm{A}^{-1} \mathbf{Z} \mathbf{Z}^{T}\left(\mathrm{~A}^{-1}\right)^{T}\right]=\sigma^{2}\left(\mathrm{~A}^{T} \mathrm{~A}\right)^{-1} .
$$

The random vector $\mathbf{X}$, defined next, is referred to as the decorrelation of $\mathbf{U}$.

$$
\mathbf{X} \triangleq \mathrm{S}^{T} \mathbf{U}
$$

where $S$ is the unitary matrix in the eigendecomposition of the positive definite matrix $\left(A^{T} A\right)^{-1}$, that is,

$$
\begin{aligned}
\left(\mathrm{A}^{T} \mathrm{~A}\right)^{-1} & =\mathrm{S} \wedge \mathrm{S}^{T}, \\
\Lambda & =\operatorname{diag}\left(\frac{1}{\mu_{1}}, \ldots, \frac{1}{\mu_{n}}\right),
\end{aligned}
$$

where $0<\mu_{1} \leq \mu_{2} \ldots \leq \mu_{n}$ are the eigenvalues of $\mathrm{A}^{T} \mathrm{~A}$ From (40) and (42), it is clear that $\mathbf{X} \sim \mathcal{N}\left(\mathbf{0}, \Sigma_{\mathbf{X}}\right)$ with covariance matrix

$$
\Sigma_{\mathbf{X}}=\mathbb{E}\left[\mathbf{X X}^{T}\right]=\mathrm{S}^{T} \Sigma_{\mathbf{U}} \mathbf{S}=\sigma^{2} \Lambda,
$$


i.e., $X_{1}, \ldots, X_{n}$ are independent zero-mean Gaussian random variables with variances

$$
\sigma_{i}^{2}=\frac{\sigma^{2}}{\mu_{i}}, \quad i \in[n] .
$$

2) Equivalence: $\mathbf{U}$ and $\mathbf{X}$ are equivalent in terms of their fundamental limits. Indeed, any excess-distortion code for $\mathbf{U}$ can be transformed, via $S$, into an excess-distortion code for $\mathbf{X}$ with the same code parameters $\epsilon, n, M, d$, and vice versa, therefore, the finite blocklength minimum achievable rates $R(n, d, \epsilon)$ for $\mathbf{U}$ and $\mathbf{X}$ are the same.

3) Distribution of eigenvalues: As we saw in (14), the eigenvalues $\sigma_{i}^{2}, i \in[n]$ of covariance matrix $\Sigma_{\mathbf{U}}$ play a key role in the computation of RDF for a Gaussian processes $\mathbf{U}$, see, e.g., [8]. Even for the Gauss-Markov source $U$ in (2), a closed-form expression for eigenvalues of $\Sigma_{\mathbf{U}}$ is unknown. The limiting distribution of the eigenvalues of $\Sigma_{\mathbf{U}}$ is given by the following result.

Theorem 3 (Asymptotic eigenvalue distribution of $\Sigma_{\mathbf{U}}$, Gray [4]). For any continuous function $F(t)$ over the interval

$$
t \in\left[\min _{w \in[-\pi, \pi]} \frac{\sigma^{2}}{g(w)}, \max _{w \in[-\pi, \pi]} \frac{\sigma^{2}}{g(w)}\right],
$$

the eigenvalues $\sigma_{i}^{2}, i \in[n]$ of $\Sigma_{\mathbf{U}}$ satisfy

$$
\lim _{n \rightarrow \infty} \frac{1}{n} \sum_{i=1}^{n} F\left(\sigma_{i}^{2}\right)=\frac{1}{2 \pi} \int_{-\pi}^{\pi} F\left[\frac{\sigma^{2}}{g(w)}\right] d w,
$$

where $g$ is defined in 24.

We develop a nonasymptotic refinement of Theorem 3, which will serve as an essential building block of our analysis.

Theorem 4 (Nonasymptotic eigenvalue distribution of $\Sigma_{\mathbf{U}}$ ). For any bounded, L-Lipschitz and non-decreasing function $F(t)$ over the interval given by (46), and for any $n \geq 1$, the eigenvalues $\sigma_{i}^{2}, i \in[n]$ of $\Sigma_{\mathbf{U}}$ satisfy

$$
\left|\frac{1}{n} \sum_{i=1}^{n} F\left(\sigma_{i}^{2}\right)-\frac{1}{2 \pi} \int_{-\pi}^{\pi} F\left[\frac{\sigma^{2}}{g(w)}\right] d w\right| \leq \frac{C_{L}}{n},
$$

where $C_{L}>0$ is a constant that depends on the Lipschitz constant $L$ and the maximum absolute value of $F$.

Proof. Appendix B-B

4) Generalized tilted information: The following Conditional Relative Entropy Minimization (CREM) problem plays a key role in our proof. Let $\mathbf{X}, \mathbf{Y}$ and $\mathbf{F}$ be random vectors of length $n$. Given the probability density functions (pdfs) $f_{\mathbf{X}}$ and $f_{\mathbf{Y}}$ of $\mathbf{X}, \mathbf{Y}$, for any $d \in\left(0, d_{\max }\right)$, the CREM problem is:

$$
\mathbb{R}(\mathbf{X}, \mathbf{Y}, d) \triangleq \inf _{\substack{P_{\mathbf{F} \mid \mathbf{X}}: \\ \mathbb{E}[\mathbf{d}(\mathbf{X}, \mathbf{F})] \leq d}} \frac{1}{n} D\left(P_{\mathbf{F} \mid \mathbf{X}}|| P_{\mathbf{Y}} \mid P_{\mathbf{X}}\right),
$$

where $D\left(P_{\mathbf{F} \mid \mathbf{X}}|| P_{\mathbf{Y}} \mid P_{\mathbf{X}}\right)$ is the conditional relative entropy, defined by

$$
\begin{aligned}
& D\left(P_{\mathbf{F} \mid \mathbf{X}} \| P_{\mathbf{Y}} \mid P_{\mathbf{X}}\right) \triangleq \\
& \int_{\mathbb{R}^{n}} f_{\mathbf{X}}(\mathbf{x}) \int_{\mathbb{R}^{n}} f_{\mathbf{F} \mid \mathbf{X}}(\mathbf{y} \mid \mathbf{x}) \log \frac{f_{\mathbf{F} \mid \mathbf{X}}(\mathbf{y} \mid \mathbf{x})}{f_{\mathbf{Y}}(\mathbf{y})} d \mathbf{y} d \mathbf{x},
\end{aligned}
$$

where $f_{\mathbf{F} \mid \mathbf{X}}$ is the conditional pdf. The CREM problem in (49) plays a crucial role in the computation of rate-distortion functions [29] [30] [31], and in non-asymptotic analyses of lossy compressors [32] [11] [13]. Operationally, it relates to lossy compression using random codewords drawn from $P_{\mathbf{Y}}$.

The optimizer $P_{\mathbf{F}^{\star} \mid \mathbf{X}}$ of CREM (49) is characterized as follows [30] [13, Sec. II.C]. For $P_{\mathbf{X F}} \mathbf{F}^{\star}$ a.e. $\mathbf{x}, \mathbf{y} \in \mathbb{R}^{n}$,

$$
\log \frac{f_{\mathbf{F}^{\star} \mid \mathbf{X}}(\mathbf{y} \mid \mathbf{x})}{f_{\mathbf{Y}}(\mathbf{y})}=\Lambda_{\mathbf{Y}}\left(\mathbf{x}, \lambda^{\star}, d\right)-\lambda^{\star} n \mathrm{~d}(\mathbf{x}, \mathbf{y})+\lambda^{\star} n d,
$$

where

$$
\lambda^{\star} \triangleq-\mathbb{R}^{\prime}(\mathbf{X}, \mathbf{Y}, d)
$$

is the negative slope of $\mathbb{R}(\mathbf{X}, \mathbf{Y}, d)$ as a function of $d$, and $\Lambda_{\mathbf{Y}}(\mathbf{x}, \lambda, d)$ is the $n$-letter generalized tilted information [13] defined for any $\lambda>0$ as

$$
\Lambda_{\mathbf{Y}}(\mathbf{x}, \lambda, d) \triangleq-\lambda n d-\log \mathbb{E}[\exp (-\lambda n \mathrm{~d}(\mathbf{x}, \mathbf{Y}))]
$$

It is known [30] that $\mathbb{R}\left(\mathbf{X}, \mathbf{Y}^{\star}, d\right)=\mathbb{R}_{\mathbf{X}}(n, d)$. Therefore, the d-tilted information (8) satisfies

$$
\jmath \mathbf{x}(\mathbf{x}, d)=\Lambda_{\mathbf{Y}^{\star}}\left(\mathbf{x}, \lambda^{\star \star}, d\right), \quad \forall \mathbf{x} \in \mathbb{R}^{n},
$$

where $\left(\mathbf{X}, \mathbf{Y}^{\star}\right)$ forms a RDF-achieving pair, and $\lambda^{\star \star}$ is given by (9). It is also easy to see that

$$
\jmath \mathbf{X}(\mathbf{x}, d)=\jmath_{\mathbf{U}}(\mathbf{u}, d),
$$

for any $\mathbf{u} \in \mathbb{R}^{n}$, and $\mathbf{x}=\mathrm{S}^{T} \mathbf{u}$ with $\mathrm{S}$ given in (43). Further properties of the CREM problem, relevant to compression of Gauss-Markov sources, are presented in Appendix B-C.

\section{NON-ASYMPTOTIC CONVERSE}

In this section, we prove the converse part of Theorem 1

Theorem 5 (Converse). For the Gauss-Markov source (2), for any excess-distortion probability $\epsilon \in(0,1)$, and for any distortion threshold $d \in\left(0, d_{\max }\right)$, the minimum achievable source coding rate satisfies

$$
R(n, d, \epsilon) \geq \mathbb{R}_{\mathbf{U}}(d)+\sqrt{\frac{V(d)}{n}} Q^{-1}(\epsilon)+O\left(\frac{1}{n}\right),
$$

where $\mathbb{R}_{\mathbf{U}}(d)$ is given in 21, and $V(d)$ is given in (33).

We present two converse proofs. The first one is based on a result on sphere covering, stated and proved in Theorem 6 This geometric proof yields a tight converse for $d \in\left(0, d_{c}\right]$, but it is not tight for $d \in\left(d_{c}, d_{\max }\right)$. The second proof, based on a general converse result of Kostina and Verdú [13], provides a tight converse for $d \in\left(0, d_{\max }\right)$.

\section{A. Geometric proof}

Theorem 6. Given $\epsilon \in(0,1)$ and $d \in\left(0, d_{\max }\right)$, the size of any $(n, M, d, \epsilon)$-excess distortion code for the Gauss-Markov source (2) must satisfy

$$
M \geq\left(\frac{r(n, \epsilon)}{d}\right)^{n / 2}
$$


where $r(n, \epsilon)$ is such that

$$
\mathbb{P}\left(G<n \cdot r(n, \epsilon) / \sigma^{2}\right)=1-\epsilon,
$$

and $G$ is a random variable distributed according to the $\chi^{2}$ distribution with $n$ degrees of freedom.

Proof of Theorem 6. The result follows via a geometric argument, illustrated in Fig. 3 Let $\mathcal{C} \subset \mathbb{R}^{n}$ be the set of codewords of an arbitrary $(n, M, d, \epsilon)$-excess distortion code, and define $\mathcal{B}(\mathbf{c}, d)$ to be the distortion $d$-ball centered at codeword $\mathbf{c} \in \mathcal{C}$ :

$$
\mathcal{B}(\mathbf{c}, d) \triangleq\left\{\mathbf{y} \in \mathbb{R}^{n}: \mathbf{d}(\mathbf{y}, \mathbf{c}) \leq d\right\} .
$$

By the definition of an $(n, M, d, \epsilon)$-excess distortion code, we know that the union of distortion $d$-balls centered at codewords in $\mathcal{C}$ has probability mass at least $1-\epsilon$, that is,

$$
\mathbb{P}\left[\mathbf{U} \in \bigcup_{\mathbf{c} \in \mathcal{C}} \mathcal{B}(\mathbf{c}, d)\right] \geq 1-\epsilon .
$$

Denote by $\mathcal{B}$ the union of the distortion $d$-balls centered at the codewords, that is,

$$
\mathcal{B} \triangleq \bigcup_{\mathbf{c} \in \mathcal{C}} \mathcal{B}(\mathbf{c}, d)
$$

For a set $\mathcal{S} \subseteq \mathbb{R}^{n}$, denote by

$$
\mathrm{A} \mathcal{S} \triangleq\{A \mathbf{s}: \mathbf{s} \in \mathcal{S}\}
$$

the linear transformation of $\mathcal{S}$ by matrix $A$. Recall from 39 that $A$ is invertible and the innovation is $\mathbf{Z}=\mathrm{AU}$. Changing variable $\mathbf{U}=A^{-1} \mathbf{Z}$ in (60) yields

$$
\mathbb{P}[\mathbf{Z} \in \mathbf{A B}] \geq 1-\epsilon
$$

Next, we give a geometric interpretation of the set $A \mathcal{B}$. Consider the set $A \mathcal{C}$, that is, the transformation of the codebook $\mathcal{C}$ by $A$. For any $\mathbf{x} \in \mathbb{R}^{n}$, notice that the set

$$
\begin{aligned}
& \mathrm{AB}\left(\mathrm{A}^{-1} \mathbf{x}, d\right)= \\
& \left\{\mathbf{x}^{\prime} \in \mathbb{R}^{n}:\left(\mathbf{x}^{\prime}-\mathbf{x}\right)^{T}\left(\mathrm{AA}^{T}\right)^{-1}\left(\mathbf{x}^{\prime}-\mathbf{x}\right) \leq n d\right\}
\end{aligned}
$$

is the set of points bounded by the ellipsoid centered at $\mathrm{x}$ with principal axes being the eigenvectors of $\mathrm{AA}^{T}$. It follows that

$$
\begin{aligned}
\mathrm{AB} & =\mathrm{A} \bigcup_{\mathbf{c} \in \mathcal{C}} \mathcal{B}(\mathbf{c}, d) \\
& =\bigcup_{\mathbf{c}^{\prime} \in \mathrm{AC}} \mathrm{AB}\left(\mathrm{A}^{-1} \mathbf{c}^{\prime}, d\right),
\end{aligned}
$$

i.e., $A \mathcal{B}$ is the union of ellipsoids centered at transformed codewords, see Fig $3 \mathrm{c}$ for an illustration of the set $A \mathcal{B}$.

Finally, the following volumetric argument completes the proof of Theorem 6 Since the volume of a union of sets is less than or equal to the sum of the sets' volumes, we have

$$
M \geq \frac{\operatorname{Vol}(\mathrm{AB})}{\operatorname{Vol}(\mathrm{AB}(\mathbf{0}, d))} .
$$

Moreover, $\operatorname{Vol}(\mathrm{AB}(\mathbf{0}, d))=\operatorname{Vol}(\mathcal{B}(\mathbf{0}, d))$ since $\operatorname{det} \mathrm{A}=1$. On the other hand, due to the spherical symmetry of the distribution of $\mathbf{Z}$, the ball $\mathcal{B}(\mathbf{0}, r(n, \epsilon))$, where $r(n, \epsilon)$ satisfies (58), has the smallest volume among all sets in $\mathbb{R}^{n}$ with probability greater than or equal to $1-\epsilon$, and so

$$
\operatorname{Vol}(\mathrm{AB}) \geq \operatorname{Vol}(\mathcal{B}(\mathbf{0}, r(n, \epsilon))) .
$$

Therefore, we can weaken 67 as

$$
M \geq \frac{\operatorname{Vol}(\mathcal{B}(\mathbf{0}, r(n, \epsilon)))}{\operatorname{Vol}(\mathcal{B}(\mathbf{0}, d))}=\left(\frac{r(n, \epsilon)}{d}\right)^{n / 2} .
$$

Remark 1. Theorem 6, for the Gauss-Markov source parallels [13, Th. 36], which applies to the Gaussian memoryless source. Both proofs rely on the volumetric method, though the proof of Theorem 6 requires additional arguments related to linear transformations of the underlying space. Theorem 6 yields the optimal second-order coding rate for the GaussMarkov source only in the low distortion regime, while an analysis of [13, Th. 36] gives the optimal optimal secondorder coding rate for Gaussian memoryless source for any distortion [13, Th. 40].

Equipped with Theorem 6 we are ready to prove the converse in Theorem 5 for $d \in\left(0, d_{c}\right]$.

Proof of Theorem 5 below critical distortion. The Gaussian approximation analysis of the bound in (57), which gives the following converse, is provided in [13, Eq. (237)-(241)]. For $\epsilon \in(0,1)$ and $d \in\left(0, d_{\max }\right)$, we have

$$
R(n, \epsilon, d) \geq \frac{1}{2} \log \frac{\sigma^{2}}{d}+\sqrt{\frac{1}{2 n}} Q^{-1}(\epsilon)+O\left(\frac{1}{n}\right) .
$$

For $\left.d \in\left(0, d_{c}\right], 470\right)$ is the same as 56, , up to the secondorder term, since $\mathbb{R}_{\mathbf{U}}(d)=\frac{1}{2} \log \frac{\sigma^{2}}{d}$ and $V(d)=1 / 2$ for $d \in\left(0, d_{c}\right]$. However, the converse in 70$)$ is not tight for $d \in\left(d_{c}, d_{\text {max }}\right)$ (even in the first order since $\mathbb{R}_{\mathbf{U}}(d)>\frac{1}{2} \log \frac{\sigma^{2}}{d}$ for $\left.d \in\left(d_{c}, d_{\max }\right)\right)$.

Remark 2. The converse (70) holds for general Gaussian autoregressive processes (19). The critical distortion $d_{c}$ of a general Gaussian process is defined by 26. The assumption 20, ensures $d_{c}>0$. The proof stays the same, except that the matrix A defined in (39) is replaced by

$$
\mathrm{A}=\left[\begin{array}{ccccc}
1 & 0 & 0 & \ldots & 0 \\
-a_{1} & 1 & 0 & \ldots & 0 \\
-a_{2} & -a_{1} & 1 & \ldots & 0 \\
\vdots & \ddots & \ddots & \ddots & \vdots \\
-a_{n-1} & \ldots & -a_{2} & -a_{1} & 1
\end{array}\right] .
$$

\section{B. Converse proof via d-tilted information}

The proof consists of analyzing the behavior of the general converse in [13], stated next.

Theorem 7 ( [13, Th. 7]). Fix $d \in\left(0, d_{\max }\right)$. Any $(n, M, d, \epsilon)$ excess-distortion code must satisfy

$$
\epsilon \geq \sup _{\gamma \geq 0} \mathbb{P}[\jmath \mathbf{X}(\mathbf{X}, d) \geq \log M+\gamma]-\exp (-\gamma) .
$$




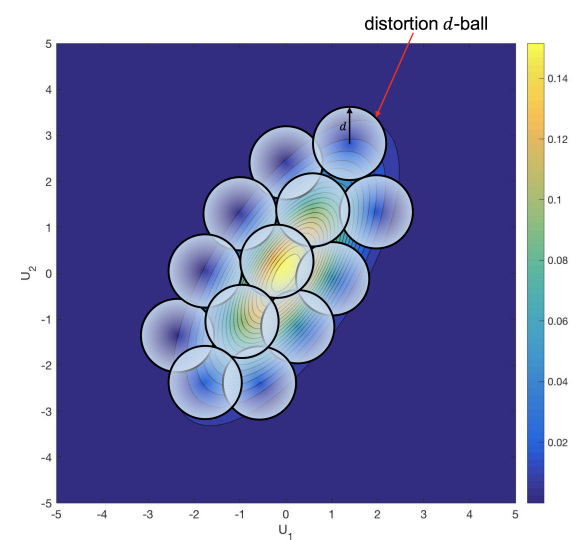

(a) The $\mathbf{U}$ space: Given $d, \epsilon$, the goal is to cover at least $1-\epsilon$ probability mass, under distribution of $\mathbf{U}$, using the least number of distortion $d$-balls.

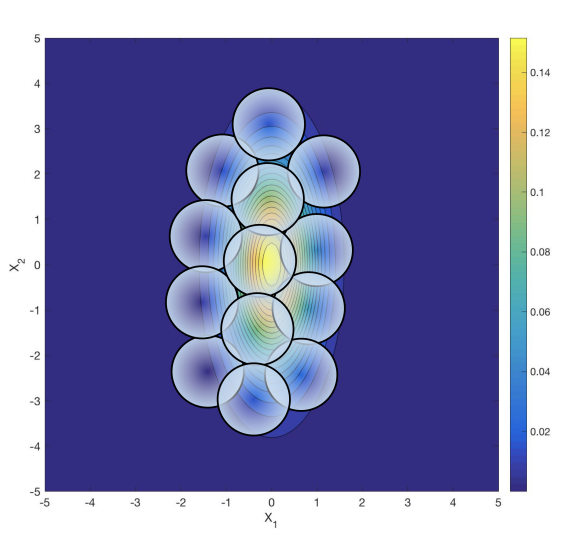

(b) The $\mathbf{X}$ space is simply a unitary transformation of the $\mathbf{U}$ space.

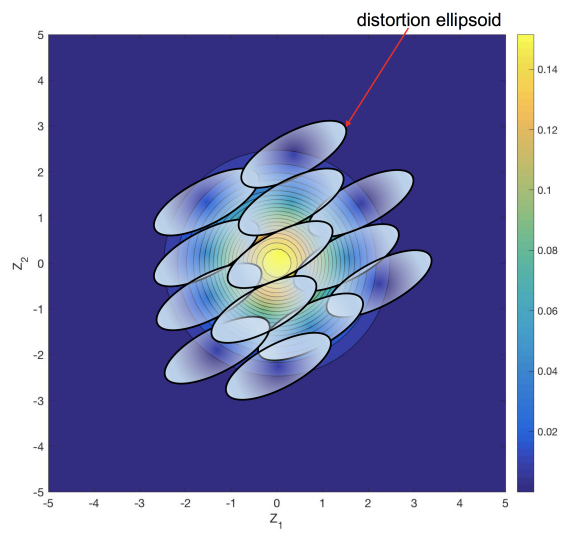

(c) The $\mathbf{Z}$ space: Given $d, \epsilon$, the goal is to cover at least $1-\epsilon$ probability mass, under distribution of $\mathbf{Z}$, using the least number of distortion ellipsoids. Note that each distortion ellipsoid has the same volume as the distortion $d$-ball due to the fact that $\operatorname{det}\left(\mathrm{AA}^{T}\right)=1$.

Fig. 3: Converse proof in figures. The contour plot in each figure shows the underlying probability distribution.

We also need the following concentration result, proved in Appendix C-A.

Theorem 8 (Difference of d-tilted information concentrates). For any $d \in\left(0, d_{\max }\right)$, let $\theta>0$ be the water level matched to $d$ via the limiting reverse waterfilling (22). Suppose we have a sequence of distortion levels $d_{n} \in\left(0, d_{\max }\right)$ such that

$$
\left|d-d_{n}\right| \leq \frac{h_{1}}{n}, \quad \forall n \geq N_{1}
$$

for some $N_{1} \in \mathbb{N}$ and constant $h_{1}>0$. Then, there exists a constant $\tilde{c} \in(0,1)$ and $N(d) \in \mathbb{N}$ such that, for any $u>\frac{2 h_{1}}{\tilde{c} \theta}$ and $n \geq N(d)$,

$$
\mathbb{P}\left[\left|\jmath \mathbf{X}(\mathbf{X}, d)-\jmath \mathbf{X}\left(\mathbf{X}, d_{n}\right)\right| \leq u\right] \geq 1-\frac{1}{n\left(\frac{\tilde{c} \theta u}{2 h_{1}}-1\right)^{2}}
$$

Proof of Theorem 5. For any fixed $d \in\left(0, d_{\max }\right)$, let $\theta>0$ be the water level matched to $d$ via the limiting reverse waterfilling (22). It is easy to check that for variable $t$ in the working interval 46, the functions $\min (\theta, t), \max \left(0, \frac{1}{2} \log \frac{t}{\theta}\right)$ and $\min \left(\frac{1}{2}, \frac{t^{2}}{2 \theta^{2}}\right)$ are bounded and non-decreasing, and have Lipschitz coefficients $L_{d}=1, L_{r}=1 /(2 \theta)$ and $L_{v}=1 / \theta$, respectively. Applying Theorem 4 to the function $\min (\theta, t)$, we conclude that there exists a constant $C_{d}>0$ such that for any $n \geq 1$,

$$
\left|d-\frac{1}{n} \sum_{i=1}^{n} \min \left(\theta, \sigma_{i}^{2}\right)\right| \leq \frac{C_{d}}{n}
$$

Define

$$
\begin{aligned}
d_{i}(\theta ; n) & \triangleq \min \left(\theta, \sigma_{i}^{2}\right), \quad \forall i \in[n] \\
d(\theta ; n) & \triangleq \frac{1}{n} \sum_{i=1}^{n} d_{i}(\theta ; n)
\end{aligned}
$$

then (75) can be rewriten as

$$
|d-d(\theta ; n)| \leq \frac{C_{d}}{n} .
$$

From (76), we know that $d(\theta ; n)$ is the distortion matched to water level $\theta$ via the $n$-th order reverse waterfilling (15) over $\sigma_{i}^{2}, \forall i \in[n]$. Notice that the difference between $d$ and $d(\theta ; n)$ decreases quickly as $n$ increases. Let $\mathbf{Y}_{\theta}^{\star}$ be such that $\left(\mathbf{X}, \mathbf{Y}_{\theta}^{\star}\right)$ form a RDF-achieving pair. To simplify notations, for each $i \in[n]$, denote by $\Lambda_{i}(\theta ; n)$ the generalized tilted information of $X_{i}$ at distortion $d_{i}(\theta ; n)$ with $\lambda=\lambda_{\theta}^{\star \star}$, i.e.,

$$
\Lambda_{i}(\theta ; n) \triangleq \Lambda_{Y_{\theta, i}^{\star}}\left(X_{i}, \lambda_{\theta}^{\star \star}, d_{i}(\theta ; n)\right), \quad \forall i \in[n],
$$

where

$$
\lambda_{\theta}^{\star \star}=-\mathbb{R}^{\prime}\left(\mathbf{X}, \mathbf{Y}_{\theta}^{\star}, d(\theta ; n)\right)
$$

and $Y_{\theta, i}^{\star} \sim \mathcal{N}\left(0, \nu_{i}^{2}\right)$ with $\nu_{i}^{2}=\sigma_{i}^{2}-d_{i}(\theta ; n)$, for all $i \in[n]$. By the independence of $Y_{\theta, 1}^{\star}, \ldots, Y_{\theta, n}^{\star}$, we have

$$
\jmath_{\mathbf{X}}(\mathbf{X}, d(\theta ; n))=\sum_{i=1}^{n} \Lambda_{i}(\theta ; n)
$$

Denote by $\mathbb{E}_{i}$ and $\mathbb{V}_{i}$ the means and variances of the random variables $\Lambda(\theta ; n, i)$, for all $i \in[n]$. As shown in Appendix B-D. for any $i \in[n]$,

$$
\begin{aligned}
\mathbb{E}_{i} & =\max \left(0, \frac{1}{2} \log \frac{\sigma_{i}^{2}}{\theta}\right), \\
\mathbb{V}_{i} & =\min \left(\frac{1}{2}, \frac{\sigma_{i}^{4}}{2 \theta^{2}}\right) .
\end{aligned}
$$

We now derive the approximation of $\mathbb{R}_{\mathbf{U}}(d)$ and $V(d)$ using the means $\mathbb{E}_{i}$ 's and variances $\mathbb{V}_{i}$ 's, respectively. Applying Theorem 4 to function $\max \left(0, \frac{1}{2} \log \frac{t}{\theta}\right)$ on (38), and to 
function $\min \left(\frac{1}{2}, \frac{t^{2}}{2 \theta^{2}}\right)$ on 37], we conclude that there exist constants $c_{r}, c_{v}>0$ (depending on $d$ only) such that

$$
\begin{array}{r}
\left|n \mathbb{R}_{\mathbf{U}}(d)-\sum_{i=1}^{n} \mathbb{E}_{i}\right| \leq c_{r}, \\
\mid \sqrt{n V(d)}-\sqrt{\sum_{i=1}^{n} \mathbb{V}_{i} \mid} \leq c_{v} .
\end{array}
$$

Next, we consider the sequence of distortion levels $\{d(\theta ; n)\}_{n=1}^{\infty}$, which satisfies the assumption (73) of Theorem 8 due to (75). Define the event

$$
\mathcal{E} \triangleq\left\{\jmath \mathbf{X}(\mathbf{X}, d) \geq \jmath \mathbf{X}(\mathbf{X}, d(\theta ; n))-\frac{4 C_{d}}{\tilde{c} \theta}\right\},
$$

where $\tilde{c}$ is the constant in Theorem 8 , and $C_{d}$ is the constant in (75). Theorem 8 implies that

$$
\mathbb{P}[\mathcal{E}] \geq 1-\frac{1}{n} .
$$

Letting $\gamma=\frac{1}{2} \log n$ in Theorem 7, we see that if an $\left(n, M, d, \epsilon^{\prime}\right)$-excess-distortion code exists, then

$$
\begin{aligned}
& \epsilon^{\prime} \geq \mathbb{P}\left[\jmath_{\mathbf{X}}(\mathbf{X}, d) \geq \log M+\frac{\log n}{2}\right]-\frac{1}{\sqrt{n}} \\
& \geq \mathbb{P}\left[\jmath \mathbf{X}(\mathbf{X}, d) \geq \log M+\frac{\log n}{2} \mid \mathcal{E}\right] \mathbb{P}[\mathcal{E}]-\frac{1}{\sqrt{n}} \\
& \geq\left(1-\frac{1}{n}\right) \mathbb{P}\left[\jmath \mathbf{X}(\mathbf{X}, d(\theta ; n)) \geq \log M+\frac{\log n}{2}+\frac{4 C_{d}}{\tilde{c} \theta}\right] \\
& \quad-\frac{1}{\sqrt{n}},
\end{aligned}
$$

where $(90)$ is by $(87)$ and by the definition of $\mathcal{E}$.

For fixed $\epsilon \in(0,1)$, choose $N \in \mathbb{N}$ large enough such that $\forall n \geq N$,

$$
\epsilon_{n} \triangleq \epsilon+\exp (-\gamma)+\frac{C_{\mathrm{BE}}}{\sqrt{n}}+\frac{1}{n} \in(0,1),
$$

where $C_{\mathrm{BE}}$ is the constant from the Berry-Esséen Theorem in Appendix 12, and let $M$ satisfy

$$
\begin{aligned}
\log M & =n \mathbb{R}_{\mathbf{U}}(d)+\sqrt{n V(d)} Q^{-1}\left(\epsilon_{n}\right) \\
& -\gamma-c_{r}-c_{v}\left|Q^{-1}\left(\epsilon_{n}\right)\right|-\frac{4 C_{d}}{\tilde{c} \theta} .
\end{aligned}
$$

Due to (84), (85) and (92), we have

$$
\log M \leq \sum_{i=1}^{n} \mathbb{E}_{i}+Q^{-1}\left(\epsilon_{n}\right) \sqrt{\sum_{i=1}^{n} \mathbb{V}_{i}}-\gamma-\frac{4 C_{d}}{\tilde{c} \theta} .
$$

Continuing the inequality in 90], we have

$$
\begin{aligned}
\epsilon^{\prime} \geq & \left(1-\frac{1}{n}\right) \mathbb{P}\left[\sum_{i=1}^{n} \Lambda_{i}(\theta ; n) \geq \sum_{i=1}^{n} \mathbb{E}_{i}+Q^{-1}\left(\epsilon_{n}\right) \sqrt{\left.\sum_{i=1}^{n} \mathbb{V}_{i}\right]}\right. \\
& \quad-\frac{1}{\sqrt{n}} \\
\geq & \left(1-\frac{1}{n}\right)\left(\epsilon_{n}-\frac{C_{\mathrm{BE}}}{\sqrt{n}}\right)-\frac{1}{\sqrt{n}} \\
\geq & \epsilon_{n}-\frac{1}{n}-\frac{1+C_{\mathrm{BE}}}{\sqrt{n}} \\
= & \epsilon
\end{aligned}
$$

where (94) is by (81) and the bound (93); (95) is by the BerryEsséen Theorem in Appendix 12, and (97) is by the choice of $\epsilon_{n}$. Consequently, for $n \geq N$, any $\left(n, M, d, \epsilon^{\prime}\right)$-excess-distortion code must satisfy $\epsilon^{\prime} \geq \epsilon$, so we must have

$$
R(n, d, \epsilon) \geq \frac{\log M}{n} .
$$

Plugging the expression 92 into 98 and applying Taylor expansion of $Q^{-1}\left(\epsilon_{n}\right)$ completes the proof of Theorem 5 .

\section{Non-ASymptotic ACHIEVABility}

In this section, we prove the achievability part of our main results, stated in Theorem 9

Theorem 9 (Achievability). Fix any $\alpha>0$. For the GaussMarkov source defined in (2), for any excess-distortion probability $\epsilon \in(0,1)$, and any distortion threshold $d \in\left(0, d_{\max }\right)$, the minimum achievable source coding rate is bounded as

$R(n, d, \epsilon) \leq \mathbb{R}_{\mathbf{U}}(d)+\sqrt{\frac{V(d)}{n}} Q^{-1}(\epsilon)+O\left(\frac{1}{(\log n)^{c \alpha} \sqrt{n}}\right)$,

where $\mathbb{R}_{\mathbf{U}}(d)$ is given in 21], and $V(d)$ is given in [33], and

$$
c \triangleq \frac{(1-a)^{2}}{20 J^{2}(1+a)^{4}},
$$

and $J$ is a universal constant defined in (355) in Appendix E-A

\section{A. A geometric idea that almost works: connections to covering number}

Dumer et al. [33] considered the problem of covering an ellipsoid using the minimum number of balls in $\mathbb{R}^{n}$, and derived lower and upper bounds on that number. Although any upper bound on the covering number implies an upper bound on $R(n, d, \epsilon)$, the upper bound on covering number in [33] is not tight enough to yield the Gaussian approximation (32). We proceed to explain how to obtain a bound on $R(n, d, \epsilon)$ from the results in [33].

An ellipsoid $E_{\mathrm{r}}^{n}$ is defined by

$$
E_{\mathbf{r}}^{n} \triangleq\left\{\mathrm{x} \in \mathbb{R}^{n}: \sum_{i=1}^{n} \frac{x_{i}^{2}}{r_{i}^{2}} \leq 1\right\},
$$

where $\mathbf{r}=\left(r_{1}, \ldots, r_{n}\right)$, and $r_{i}>0$ gives the half the length of the $i$-axis of $E_{\mathbf{r}}^{n}$. We say that a subset $\mathcal{M}_{d} \subset \mathbb{R}^{n}$ is a $d$-covering $\mathrm{g}^{4}$ of $E_{\mathbf{r}}^{n}$ if

$$
E_{\mathbf{r}}^{n} \subseteq \bigcup_{\mathbf{y} \in \mathcal{M}_{d}} \mathcal{B}(\mathbf{y}, d)
$$

where $\mathcal{B}(\mathbf{y}, d)$ is the $d$-ball centered at $\mathbf{y} \in \mathbb{R}^{n}$, defined in (59). The covering number $\mathcal{N}(n, d)$ of an ellipsoid $E_{\mathbf{r}}^{n}$ is defined as the size of its minimal $d$-covering. The $d$-entropy $H_{d}\left(E_{\mathbf{r}}^{n}\right)$ is the logarithm of the covering number

$$
H_{d}\left(E_{\mathbf{r}}^{n}\right) \triangleq \log \mathcal{N}(n, d) .
$$

\footnotetext{
${ }^{4}$ In [33], the term $\varepsilon$-covering was used instead of $d$-covering used here. The
} relation is $\varepsilon=\sqrt{n d}$. 
The result in [33, Th. 2] states that

$$
H_{d}\left(E_{\mathbf{r}}^{n}\right)=K_{d}+o\left(K_{d}\right),
$$

where

$$
K_{d} \triangleq \sum_{i: r_{i}^{2}>n d} \frac{1}{2} \log \frac{r_{i}^{2}}{n d} .
$$

Despite the similarity of (105) and the reverse waterfilling (14), the result in (104) is not strong enough to recover (14) unless $d \leq d_{c}$.

In our problem, let $\mathbf{X}$ be the decorrelation of $\mathbf{U}$ in $(41$, then $X_{1}, \ldots, X_{n}$ are independent zero-mean Gaussian distributed with variances being $\sigma_{i}^{2}$ defined in 45 . Random vector $\mathbf{X}$ concentrates around an ellipsoid with probability mass at least $1-\epsilon$. Using the bound on the number of $d$-balls necessary to cover that ellipsoid in (104) and the Berry-Esséen theorem, one can show that for any $\epsilon \in(0,0.5)$ and $d \in\left(0, d_{c}\right]$,

$$
R(n, d, \epsilon) \leq \frac{1}{2} \log \frac{\sigma^{2}}{d}+\frac{Q^{-1}(\epsilon)}{\sqrt{2 n}}+o(1),
$$

which is first-order optimal, but not second-order optimal (due to the $o(1)$ term), compared to Theorem 1 for $d \in\left(0, d_{c}\right]$ :

$$
R(n, d, \epsilon)=\frac{1}{2} \log \frac{\sigma^{2}}{d}+\frac{Q^{-1}(\epsilon)}{\sqrt{2 n}}+o\left(\frac{1}{\sqrt{n}}\right) .
$$

Although strenghthening (104) to $H_{d}\left(E_{\mathbf{r}}^{n}\right)=K_{d}+o\left(\sqrt{K_{d}}\right)$ would yield the $\leq$ (achievability) direction of the Gaussian approximation (32) in the low distortion regime, we will not pursue this approach here. Instead, we prove (32) via tilted information.

\section{B. Our main ideas: a maximum likelihood estimator}

The approach to the proof is inspired by the work of Kostina and Verdú [13, Th. 12], where the same problem was addressed for stationary and memoryless sources. However, the proof techniques in [13] cannot be directly applied to the GaussMarkov source. We need the following lemmas.

Lemma 1 (Random coding bound, [13, Cor. 11]). Let $\mathbf{X}$ be the decorrelation of $\mathbf{U}$ defined in 41 . There exists an $(n, M, d, \epsilon)$ excess distortion code with

$$
\epsilon \leq \inf _{P_{\mathbf{Y}}} \mathbb{E}_{\mathbf{X}}\left[e^{-M P_{\mathbf{Y}}(\mathcal{B}(\mathbf{X}, d))}\right],
$$

where the infimum is over all pdf's $P_{\mathbf{Y}}$ on $\mathbb{R}^{n}$ with $\mathbf{Y}$ independent of $\mathbf{X}$.

Lemma 2. Fix any $\alpha>0$. For any $d \in\left(0, d_{\max }\right)$ and $\epsilon \in$ $(0,1)$, there exists $N(d, \epsilon, \alpha) \in \mathbb{N}$ and constant $K>0$ such that, for any $n \geq N(d, \epsilon, \alpha)$,

$$
\begin{aligned}
\mathbb{P}\left[\log \frac{1}{P_{\mathbf{Y}^{\star}}(\mathcal{B}(\mathbf{X}, d))} \leq \jmath \mathbf{X}(\mathbf{X}, d)\right. & \left.+c_{1} \log ^{q} n+c_{2}\right] \\
& \geq 1-\frac{K}{(\log n)^{c \alpha}},
\end{aligned}
$$

where $\mathbf{X}$ is the decorrelation of $\mathbf{U}$ in (41), and $\left(\mathbf{X}, \mathbf{Y}^{\star}\right)$ forms a RDF-achieving pair, and $q>1, c_{1}>0, c_{2}$ are constants, specified in the proof.
Proof. Appendix $\overline{\mathrm{D}-\mathrm{A}}$

Similar to [13. Lem. 2], applicable to stationary memoryless sources, Lemma 2 relates the probability of the distortion balls to d-tilted information for the Gauss-Markov source, but our Lemma 2 is slightly weaker. First, inside the probability on the left side of (109), we need $\log ^{q} n, q>1$, instead of $\log n$ in [13, Lem. 2]. Second, the bound on the right side of (109) goes to 1 in the order of $1 /$ poly $(\log n)$, while it is $\Theta(1 / \sqrt{n})$ in [13. Lem. 2]. However, this weakness only affects the thirdorder term in the Gaussian approximation. We also emphasize that the proof of our Lemma 2 necessitates a number of new tools. The MLE-typical set, presented next, is one of such tools.

In the following, we first analyze an estimation problem, and then define a proxy random variable $\hat{\mathbf{X}}$, which takes over the role of the product of empirical distributions in previous analyses for the i.i.d. case [11], [13, Eq. (270)]. Finally, we define an MLE-typical set and present its properties.

1) Maximum likelihood estimator: Suppose we observe a block of samples $\mathbf{u}=\left(u_{1}, \ldots, u_{n}\right)$ drawn from the Gauss-Markov model (2). We are interested in estimating the parameter $a$, assuming the variance $\sigma^{2}$ of the innovations $Z_{i}$ 's is known. The maximum likelihood estimator (MLE), denoted by $\hat{a}(\mathbf{u})$, is given by the following lemma.

Lemma 3 (MLE). Consider the Gauss-Markov source (2) with unknown a and known variance of $Z_{i}$ 's. Given observation $\mathbf{u}=\left(u_{1}, \ldots, u_{n}\right)$ produced by the Gauss-Markov source, the maximum likelihood estimator of a is

$$
\hat{a}(\mathbf{u})=\frac{\sum_{i=2}^{n} u_{i-1} u_{i}}{\sum_{i=2}^{n} u_{i-1}^{2}} .
$$

Proof. The random vector $\left(U_{1}, U_{2}-a U_{1}, \ldots, U_{n}-a U_{n-1}\right)$ is distributed according to $\mathcal{N}\left(0, \sigma^{2} I\right)$. Let $p_{a}(\cdot)$ be the probability density function of $\mathbf{U}$ with parameter $a$, then

$$
\begin{aligned}
& \hat{a}(\mathbf{u}) \\
\triangleq & \arg \max _{a} p_{a}(\mathbf{u}) \\
= & \arg \max _{a} \prod_{i=1}^{n} \frac{1}{\sqrt{2 \pi \sigma^{2}}} e^{-\frac{1}{2 \sigma^{2}}\left(u_{i}-a u_{i-1}\right)^{2}} \\
= & \arg \min _{a} \sum_{i=2}^{n}\left(u_{i}-a u_{i-1}\right)^{2}+u_{1}^{2} \\
= & \arg \min _{a}\left(\sum_{i=2}^{n} u_{i-1}^{2}\right) a^{2}-\left(2 \sum_{i=2}^{n} u_{i-1} u_{i}\right) a+\sum_{i=1}^{n} u_{i}^{2} \\
= & \frac{\sum_{i=2}^{n} u_{i-1} u_{i}}{\sum_{i=2}^{n} u_{i-1}^{2}}
\end{aligned}
$$

where (113) is by collecting the terms in the exponent, and 115 is by minimizing the quadratic function of $a$.

Crucially, the following theorem establishes that the estimation error of the MLE decays exponentially in $n$. 
Theorem 10 (Estimation error of the MLE). Consider the Gauss-Markov source (2) with unknown a and known variance of $Z_{i}$ 's. For any $\eta$ satisfying

$$
0<\eta<\frac{2(1+a)^{2}}{(1-a) \pi}
$$

and any $n \geq \frac{3-a}{1-a}$, the ML estimate of a satisfies

$$
\mathbb{P}[|\hat{a}(\mathbf{U})-a|>\eta] \leq 2 \max \left\{e^{2} e^{-c n \eta^{2}}, 2 e^{-\frac{n}{64}}\right\},
$$

where $c$ is the constant defined in 100 .

Proof. Appendix E-A

Theorem 10 implies that the probability mass of the set of the sequences $\mathbf{u}=\left(u_{1}, \ldots, u_{n}\right)$ with the estimation error $|\hat{a}(\mathbf{u})-a|$ exceeding $\eta$ decays exponentially in $n$. This motivates the definition of an MLE-typical set, which we discuss next.

2) Proxy Gaussian random variables and an MLE-typical set: For any source outcome $\mathbf{u}$, let $\mathbf{x}=\mathrm{S}^{T} \mathbf{u}$ be the decorrelation (41), we construct a proxy random variable, denoted by $\hat{\mathbf{X}}(\mathbf{u})$, to $\mathbf{X}$. We first define the proxy variances $\hat{\sigma}_{i}^{2}(\mathbf{u}), i \in[n]$ as

$$
\hat{\sigma}_{i}^{2}(\mathbf{u}) \triangleq \frac{\sigma^{2}}{1+\hat{a}(\mathbf{u})^{2}-2 \hat{a}(\mathbf{u}) \cos (i \pi /(n+1))} .
$$

Then $\hat{\mathbf{X}}(\mathbf{u})$ is the zero-mean Gaussian random vector:

$$
\hat{\mathbf{X}}(\mathbf{u}) \sim \mathcal{N}\left(\mathbf{0}, \operatorname{diag}\left(\hat{\sigma}_{1}^{2}(\mathbf{u}), \ldots, \hat{\sigma}_{n}^{2}(\mathbf{u})\right)\right) .
$$

To simplify notations, when there is no ambiguity, we will write $\hat{\mathbf{X}}$ and $\hat{\sigma}_{i}^{2}, i \in[n]$ for $\hat{\mathbf{X}}(\mathbf{u})$ and $\hat{\sigma}_{i}^{2}(\mathbf{u}), i \in[n]$, respectively.

As will be apparent from (176) in Appendix B-A the formula in (118) essentially gives the eigenvalues of the covariance matrix of $\mathbf{U}$ (and equivalently, of $\mathbf{X}$ ). For a typical $\mathbf{u}, \hat{a}(\mathbf{u})$ is close to $a, \hat{\sigma}_{i}^{2}$ is close to $\sigma_{i}^{2}$, and the distribution of $\hat{\mathbf{X}}(\mathbf{u})$ is close to that of $\mathbf{X}$. The accuracy of these approximations is quantified in Theorem 11 below.

The proxy random variable $\hat{\mathbf{X}}(\mathbf{u})$ is central to our achievability proof. In particular, in the proof of Lemma 2 , the proxy distribution $P_{\hat{\mathrm{X}}}$ takes over the role of the product of empirical distributions in previous analyses for the i.i.d. case in [11] [13 Eq. (270)]. There, $P_{\hat{\mathrm{X}}}$ is taken to be $P_{\hat{\mathrm{X}}} \times \ldots \times P_{\hat{\mathrm{X}}}$, where $P_{\hat{\mathrm{X}}}$ is the empirical distribution of $x^{n} \in \mathcal{X}^{n}$ (the mass the emprical distribution assigns to $x \in \mathcal{X}$ is equal to the number of times $x$ appears in the vector $x^{n}$ ). Therefore, $P_{\hat{\mathrm{X}}}$ is an estimate of the source distribution, given a single block of observations of length $n$ and knowing that $x^{n}$ is drawn from the stationary and memoryless source distribution. Similarly, our $P_{\hat{\mathrm{X}}}$ serves as an estimate of the source distribution based on the knowledge of $x^{n}$ and the fact that $x^{n}$ is drawn from a family of Gauss-Markov distributions parameterized by $a$. This is the key difference between the proof of Lemma 2 (applicable to the Gauss-Markov source) and [13, Lem. 2] (applicable to stationary memoryless sources).

Consider the optimization problem $\mathbb{R}\left(\hat{\mathbf{X}}, \mathbf{Y}^{\star}, d\right)$ in 49, with $\hat{\mathbf{X}}$ in 1119 , and $\mathbf{Y}^{\star}$ such that $\left(\mathbf{X}, \mathbf{Y}^{\star}\right)$ forms a $\mathrm{RDF}$ achieving pair, where $\mathbf{X}$ is the decorrelation of $\mathbf{U}$ (41). Let
$\hat{\mathbf{F}}^{\star}$ be the optimizer of $\mathbb{R}\left(\hat{\mathbf{X}}, \mathbf{Y}^{\star}, d\right)$. For $\mathbf{x} \in \mathbb{R}^{n}$, define $m_{i}(\mathbf{x}), i \in[n]$ are defined as

$$
m_{i}(\mathbf{x}) \triangleq \mathbb{E}\left[\left(\hat{F}_{i}^{\star}-x_{i}\right)^{2} \mid \hat{X}_{i}=x_{i}\right] .
$$

Definition 2 (MLE-typical set). For any $d \in\left(0, d_{\max }\right)$, some constants $\alpha, p>0$, and any $n \in \mathbb{N}$, define $\mathcal{T}(n, \alpha, p)$ to be the set of vectors $\mathbf{u} \in \mathbb{R}^{n}$ that satisfy the following conditions:

$$
\begin{aligned}
|\hat{a}(\mathbf{u})-a| & \leq \sqrt{\frac{\alpha \log \log n}{n}}, \\
\left|\frac{1}{n} \sum_{i=1}^{n} m_{i}(\mathbf{x})-d\right| & \leq p \sqrt{\frac{\alpha \log \log n}{n}}, \\
\left|\frac{1}{n} \sum_{i=1}^{n}\left(\frac{x_{i}^{2}}{\sigma_{i}^{2}}\right)^{k}-(2 k-1) ! !\right| & \leq 2, \text { for } k=1,2,3,
\end{aligned}
$$

where $\mathbf{x}=\mathrm{S}^{T} \mathbf{u}$, and $m_{i}(\mathbf{x}), i \in[n]$ are functions of $\mathbf{x}$ defined in (120).

The condition (121) requires that $\mathbf{u} \in \mathcal{T}(n, \alpha, p)$ should yield small estimation error, and this condition holds with high probability (w.h.p.) due to Theorem 10 . We will explain condition (122) in Appendix D-D. In condition (123), due to 444, for any $i \in[n]$ and $k=1,2,3$, we have $\frac{X_{i}}{\sigma_{i}} \sim \mathcal{N}(0,1)$ and

$$
\mathbb{E}\left[\left(\frac{X_{i}^{2}}{\sigma_{i}^{2}}\right)^{k}\right]=(2 k-1) ! ! .
$$

Condition (123) bounds variations of $\mathbf{X}$, up to sixth moments, and this condition holds with high probability by the BerryEsséen theorem. Theorem 11, stated next, summarizes the properties of the typical set needed in the proof of Lemma 2

Theorem 11 (Properties of the MLE-typical set). For any $d \in\left(0, d_{\max }\right)$ and constant $\alpha>0$, let $p$ be a sufficiently large constant (specifically, $p \geq 2$ (298)), then

- the probability mass of $\mathcal{T}(n, \alpha, p)$ is large: there exists a constant $A_{1}>0$ and $N(d, \alpha) \in \mathbb{N}$ such that for any $n \geq N(d, \alpha)$,

$$
\mathbb{P}[\mathbf{U} \in \mathcal{T}(n, \alpha, p)] \geq 1-\frac{A_{1}}{(\log n)^{c \alpha}},
$$

where the constant $c$ is defined in 100 .

- the proxy variances are good approximations: there exists a constant $A_{2}>0$ and $N \in \mathbb{N}$ such that for any $\mathbf{u} \in$ $\mathcal{T}(n, \alpha, p)$, and any $n \geq N$, and any $i \in[n]$, it holds that

$$
\left|\hat{\sigma}_{i}^{2}(\mathbf{u})-\sigma_{i}^{2}\right| \leq A_{2} \sqrt{\frac{\alpha \log \log n}{n}} .
$$

- Let $\theta>0$ be the water level matched to $d$ via the limiting reverse waterfilling (22). There exists $N \in \mathbb{N}$ such that for any $\mathbf{u} \in \mathcal{T}(n, \alpha, p)$, for any $n \geq N$, and any $i \in[n]$, it holds that

$$
\left|\hat{\lambda}^{\star}(\mathbf{x})-\lambda^{\star \star}\right| \leq \frac{9 A_{2}}{4 \theta^{2}} \sqrt{\frac{\alpha \log \log n}{n}},
$$

where $\mathbf{x}=\mathrm{S}^{T} \mathbf{u}, \lambda^{\star \star}$ is given by (9), and

$$
\hat{\lambda}^{\star}(\mathbf{x})=-\mathbb{R}^{\prime}\left(\hat{\mathbf{X}}, \mathbf{Y}^{\star}, d\right),
$$


$\mathbf{X}$ is the decorrelation of $\mathbf{U}(41),\left(\mathbf{X}, \mathbf{Y}^{\star}\right)$ forms a $R D F$ achieving pair, and $\hat{\mathbf{X}}$ is the proxy Gaussian random variable defined in (119).

Proof. Appendix D-B

The proof of Lemma 2 relies heavily on the properties of the typical set $\mathcal{T}(n, \alpha, p)$ in Theorem 11, see Appendix D-A In the following, we present the proof of Theorem 9 by leveraging Lemma 1 and Lemma 2

\section{Achievability proof via tilted information}

Proof of Theorem 9 The proof is based on the random coding bound Lemma 1 and Lemma 2 Fix any $d \in\left(0, d_{\max }\right)$ and $\epsilon \in(0,1)$, and let $\theta>0$ be the water level matched to $d$ via the limiting reverse waterfilling (22). We reuse the notations in $(75)$ - 85 . Similar to the event $\mathcal{E}$ in 886 , we define the event $\mathcal{F}$ as

$$
\mathcal{F} \triangleq\left\{\jmath \mathbf{X}(\mathbf{X}, d) \leq \jmath \mathbf{X}(\mathbf{X}, d(\theta ; n))+\frac{4 C_{d}}{\tilde{c} \theta}\right\} .
$$

Theorem 8 implies that

$$
\mathbb{P}[\mathcal{F}] \geq 1-\frac{1}{n} .
$$

Choose $N \in \mathbb{N}$ large enough such that for any $n \geq N$,

$$
\epsilon_{n} \triangleq \epsilon-\frac{C_{\mathrm{BE}}+1}{\sqrt{n}}-\frac{K}{(\log n)^{c \alpha}}-\frac{1}{n} \in(0,1) .
$$

Choose $M$ as

$$
\begin{aligned}
\log M & \triangleq n \mathbb{R}_{\mathbf{U}}(d)+\sqrt{n V(d)} Q^{-1}\left(\epsilon_{n}\right)+\log \frac{\log n}{2} \\
& +c_{1} \log ^{q} n+c_{2}+c_{r}+c_{v}\left|Q^{-1}\left(\epsilon_{n}\right)\right|+\frac{4 C_{d}}{\tilde{c} \theta},
\end{aligned}
$$

where $q>1, c_{1}>0, c_{2}$ are constants given in Lemma 2, and $c_{r}, c_{v}$ are positive constants given in (84) and 85 . Define the random variable $G_{n}$ as

$$
G_{n} \triangleq \log M-\jmath \mathbf{X}(\mathbf{X}, d(\theta ; n))-c_{1} \log ^{q} n-c_{2}-\frac{4 C_{d}}{\tilde{c} \theta},
$$

where $\jmath_{\mathbf{X}}(\mathbf{X}, d(\theta ; n))$ is in 81 . By the bounds in 84, , 85), and (133), we have

$$
\begin{array}{r}
G_{n} \geq \sum_{i=1}^{n} \mathbb{E}_{i}+Q^{-1}\left(\epsilon_{n}\right) \sqrt{\sum_{i=1}^{n} \mathbb{V}_{i}}-\jmath \mathbf{X}(\mathbf{X}, d(\theta ; n)) \\
+\log \frac{\log n}{2} .
\end{array}
$$

Define event $\mathcal{G}$ as

$$
\mathcal{G} \triangleq\left\{G_{n}<\log \frac{\log n}{2}\right\} .
$$

By (134), (81) and the Berry-Esséen Theorem, we have

$$
\begin{aligned}
\mathbb{P}[\mathcal{G}] & \leq \mathbb{P}\left[\jmath \mathbf{X}(\mathbf{X}, d(\theta ; n))-\sum_{i=1}^{n} \mathbb{E}_{i} \geq Q^{-1}\left(\epsilon_{n}\right) \sqrt{\sum_{i=1}^{n} \mathbb{V}_{i}}\right] \\
& \leq \epsilon_{n}+\frac{C_{\mathrm{BE}}}{\sqrt{n}} .
\end{aligned}
$$

Define the event

$$
\begin{aligned}
& \mathcal{L} \triangleq\left\{\log \frac{1}{P_{\mathbf{Y}^{\star}}(\mathcal{B}(\mathbf{X}, d))} \leq \log M-G_{n}\right\} \\
&=\left\{\log \frac{1}{P_{\mathbf{Y}^{\star}}(\mathcal{B}(\mathbf{X}, d))} \leq \jmath \mathbf{X}(\mathbf{X}, d(\theta ; n))+\right. \\
&\left.c_{1} \log ^{q} n+c_{2}+\frac{4 C_{d}}{\tilde{c} \theta}\right\} .
\end{aligned}
$$

Combining Lemma 2 and 130 yields

$$
\mathbb{P}[\mathcal{L}] \geq 1-\frac{1}{n}-\frac{K}{(\log n)^{c \alpha}} .
$$

Indeed, denoting the probability on the left-hand side of (109) by $\mathbb{P}[\mathcal{H}]$, we have

$$
\begin{aligned}
\mathbb{P}[\mathcal{H}] & =\mathbb{P}[\mathcal{H} \mid \mathcal{F}] \mathbb{P}[\mathcal{F}]+\mathbb{P}\left[\mathcal{H} \mid \mathcal{F}^{c}\right] \mathbb{P}\left[\mathcal{F}^{c}\right] \\
& \leq \mathbb{P}[\mathcal{L}]+\frac{1}{n}
\end{aligned}
$$

where (140) holds since the intersection of $\mathcal{H}$ and $\mathcal{F}$ is contained in $\mathcal{L}$.

Now, we have all the ingredients needed to prove Theorem 9 In Lemma 1. choose $\mathbf{Y}$ to be $\mathbf{Y}^{\star}$ such that $\left(\mathbf{X}, \mathbf{Y}^{\star}\right)$ forms a RDF-achieving pair. Then, there exists an $\left(n, M, d, \epsilon^{\prime}\right)$-excess distortion code with

$$
\begin{aligned}
\epsilon^{\prime} \leq & \mathbb{E}_{\mathbf{X}}\left[e^{-M P_{\mathbf{Y}^{\star}}(\mathcal{B}(\mathbf{X}, d))}\right] \\
= & \mathbb{E}_{\mathbf{X}}\left[e^{-M P_{\mathbf{Y}^{\star}}(\mathcal{B}(\mathbf{X}, d))} \mid \mathcal{L}\right] \mathbb{P}[\mathcal{L}]+ \\
& \mathbb{E}_{\mathbf{X}}\left[e^{\left.-M P_{\mathbf{Y}^{\star}(\mathcal{B}(\mathbf{X}, d))} \mid \mathcal{L}^{c}\right]} \mathbb{P}\left[\mathcal{L}^{c}\right]\right. \\
\leq & \mathbb{E}_{\mathbf{X}}\left[e^{-e^{G_{n}}}\right]+\frac{K}{(\log n)^{c \alpha}}+\frac{1}{n} \\
= & \mathbb{E}_{\mathbf{X}}\left[e^{-e^{G_{n}}} \mid \mathcal{G}\right] \mathbb{P}[\mathcal{G}]+\mathbb{E}_{\mathbf{X}}\left[e^{-e^{G_{n}}} \mid \mathcal{G}^{c}\right] \mathbb{P}\left[\mathcal{G}^{c}\right]+ \\
\leq & \quad \frac{K}{(\log n)^{c \alpha}}+\frac{1}{n}(\mathcal{G})+\frac{1}{\sqrt{n}} \mathbb{P}\left(\mathcal{G}^{c}\right)+\frac{K}{(\log n)^{c \alpha}}+\frac{1}{n} \\
\leq & \epsilon_{n}+\frac{C_{\mathrm{BE}}+1}{\sqrt{n}}+\frac{K}{(\log n)^{c \alpha}}+\frac{1}{n} \\
= & \epsilon
\end{aligned}
$$

where (143) by weakening (108) using $\mathbf{Y}=\mathbf{Y}^{\star}$; (144) is by law of total expectation; (145) holds since (140) and that $\mathcal{L}$ implies $M P_{\mathbf{Y}^{\star}}(\mathcal{B}(\mathbf{X}, d)) \geq e^{G_{n}} ; 146$ is again by law of total expectation; (147) holds since $e^{-e^{G_{n}}} \leq 1$ and $\mathcal{G}^{c}$ implies $\left.e^{-e^{G_{n}}} \leq \frac{1}{\sqrt{n}} ; 148\right)$ is by 137); and 149 is by the choice of $\epsilon_{n}$ in 131. Consequently, since there exists an $\left(n, M, d, \epsilon^{\prime}\right)$ excess distortion code with $\epsilon^{\prime} \leq \epsilon$, we must have

$$
R(n, d, \epsilon) \leq \frac{\log M}{n},
$$

where $\log M$ is given by (132). Similar to the converse proof, plugging (132) into (150), and then using the Taylor expansion of $Q^{-1}\left(\epsilon_{n}\right)$ yields 99 . 


\section{CONCLUSION}

In this paper, we derived the reverse waterfilling characterization (33) of the dispersion for lossy compression of the GaussMarkov source (2) (Theorem 1). This is the first dispersion result for lossy compression of sources with memory. In doing so, we developed several novel technical tools, which are highlighted below.

- We derived the expression for the limiting variance of the d-tilted information for the Gauss-Markov source (2) in Theorem 2 Its proof relies on our parametric representation for the d-tilted information, presented in Lemma 7 in Appendix B-D

- Theorem 4 presented a nonasymptotic refinement of Gray's result [4] (Theorem 3) on the eigenvalue distribution of the covariance matrix of the random vector $\mathbf{U}$ from the Gauss-Markov source (2). The key tool we developed to prove Theorem 4 is Lemma 4 in Appendix B-A, which is a sharp bound relating the eigenvalues of two sequences of symmetric tridiangonal matrices.

- The maximum likelihood estimator $\hat{a}(\mathbf{u})$, defined in Lemma 3 and analyzed in Theorem 10 , is of independent interest. For example, the estimator $\hat{a}(\mathbf{u})$ could be used to further estimate other quantities (e.g. estimating ratedistortion functions from source outcomes) of the source using observations $\mathbf{u}$. Besides, the error bounds in Theorem 10 rely on a concentration inequality of multilinear polynomials in independent random variables. That tool applies beyond Gauss-Markov processes, which means that our approach can be applied to other sources with memory.

- To prove achievability, we constructed a typical set in Definition 2 based on a maximum likelihood estimator. This idea of constructing typical sets via estimators could also find its use in other problems.

Finally, we discuss several open problems.

- The dispersion for Gauss-Markov source with $|a| \geq 1$ is unknown. This paper treats the stationary GaussMarkov source case, i.e., $|a|<1$. The case $|a| \geq 1$ is fundamentally different, since that source is nonstationary. The rate-distortion functions for nonstationary Gaussian autoregressive processes were first derived by Gray 14 Eq. (22a), (22b)] in 1970, and later in 1980 by Hashimoto and Arimoto [25, Eq.(6a), (6b)] in an equivalent but distinct form, and the equivalence was shown by Gray and Hashimoto [26] in 2008. Gray's reverse waterfilling [4, Eq. (22a), (22b)] is different from Kolmogorov's reverse waterfilling (21) in the nonstationary case, where the later does not apply. Therefore, in order to characterize the dispersion for the case $|a| \geq 1$, one would need to use Gray's reverse waterfilling [4. Eq. (22a), (22b)] for $\mathbb{R}_{\mathbf{U}}(d)$.

- A natural generalization of this work would be to consider the dispersion for the general stationary Gaussian autoregressive processes (19). The geometric converse proof in Section III already yields a converse bound on $R(n, d, \epsilon)$, which is tight in the low distortion regime $d \in\left(0, d_{c}\right]$ in the first-order term; we conjecture it is also tight in the second-order term. A possible way to show a matching achievability bound for the Gaussian autoregressive source of order $m$, inspired by the estimation idea in this paper, is to analyze an estimator which estimates the vector $\mathbf{a}=\left(a_{1}, \ldots, a_{m}\right)$ in (19) instead of the scalar $a$ in the Gauss-Markov source case. To deal with large distortion, i.e. $d>d_{c}$, sharp bounds on eigenvalues of $A^{T} A$ with $A$ given by (71) need to be derived, similar to Lemma 4 in Appendix B-A, and the tools in Appendix B-A might be useful.

- A fundamental problem left open is how widely the limiting formula for the dispersion

$$
V(d)=\lim _{n \rightarrow \infty} \frac{1}{n} \operatorname{Var}\left[\jmath_{\mathbf{X}}(\mathbf{X}, d)\right]
$$

applies. Theorem 1 and Theorem 2 established its validity for the Gauss-Markov source. We conjecture that it continues to apply whenever the central limit theorem type of results can be derived for $\jmath_{\mathbf{X}}(\mathbf{X}, d)$.

\section{APPENDIX A}

\section{A. A roadmap of the paper}

The relations of our main theorems, lemmas, corollaries are presented in Fig. 4 .

\section{B. Classical theorems}

Theorem 12 (Berry-Esséen Theorem, e.g. [34, Chap. 16.5]). Let $W_{1}, \ldots, W_{n}$ be a collection of independent zero-mean random variables with variances $V_{i}^{2}>0$ and finite third absolute moment $T_{i} \triangleq \mathbb{E}\left[\left|W_{i}\right|^{3}\right]<+\infty$. Define the average variance $V^{2}$ and average third absolute moment $T$ as

$$
V^{2} \triangleq \frac{1}{n} \sum_{i=1}^{n} V_{i}^{2}, \quad T \triangleq \frac{1}{n} \sum_{i=1}^{n} T_{i} .
$$

Then for $n \in \mathbb{N}$, we have

$$
\sup _{t \in \mathbb{R}}\left|\mathbb{P}\left\{\frac{1}{V \sqrt{n}} \sum_{i=1}^{n} W_{i}<t\right\}-\Phi(t)\right| \leq \frac{6 T}{V^{3} \sqrt{n}},
$$

where $\Phi$ is the cdf of the standard normal distribution $\mathcal{N}(0,1)$.

Remark 3. Since in this paper, we only consider random variables $W_{i}$ 's with bounded $p$-th moment for any finite $p$, it is easy to check that there exists a constant $C_{\mathrm{BE}}>0$ such that

$$
\sup _{t \in \mathbb{R}}\left|\mathbb{P}\left\{\frac{1}{V \sqrt{n}} \sum_{i=1}^{n} W_{i}<t\right\}-\Phi(t)\right| \leq \frac{C_{\mathrm{BE}}}{\sqrt{n}} .
$$

While the constant $C_{\mathrm{BE}}$ depends on the random variables, to simplify notations, we will use $C_{\mathrm{BE}}$ in all applications of the Berry-Esséen Theorem.

We also record the following direct application of Chernoff's bound to a $\chi^{2}$ random variable.

Theorem 13 (Chernoff's bound on $\chi^{2}$ random variables). Let $G_{1}, \ldots, G_{n}$ be i.i.d. Gaussian random variables and $G_{1} \sim$ $\mathcal{N}\left(0, \sigma^{2}\right)$. For any $t \in(0,1)$, it holds that

$$
\mathbb{P}\left[\left|\sum_{i=1}^{n} G_{i}^{2}-n \sigma^{2}\right| \geq t n \sigma^{2}\right] \leq 2 e^{-\frac{t^{2} n}{8}} .
$$




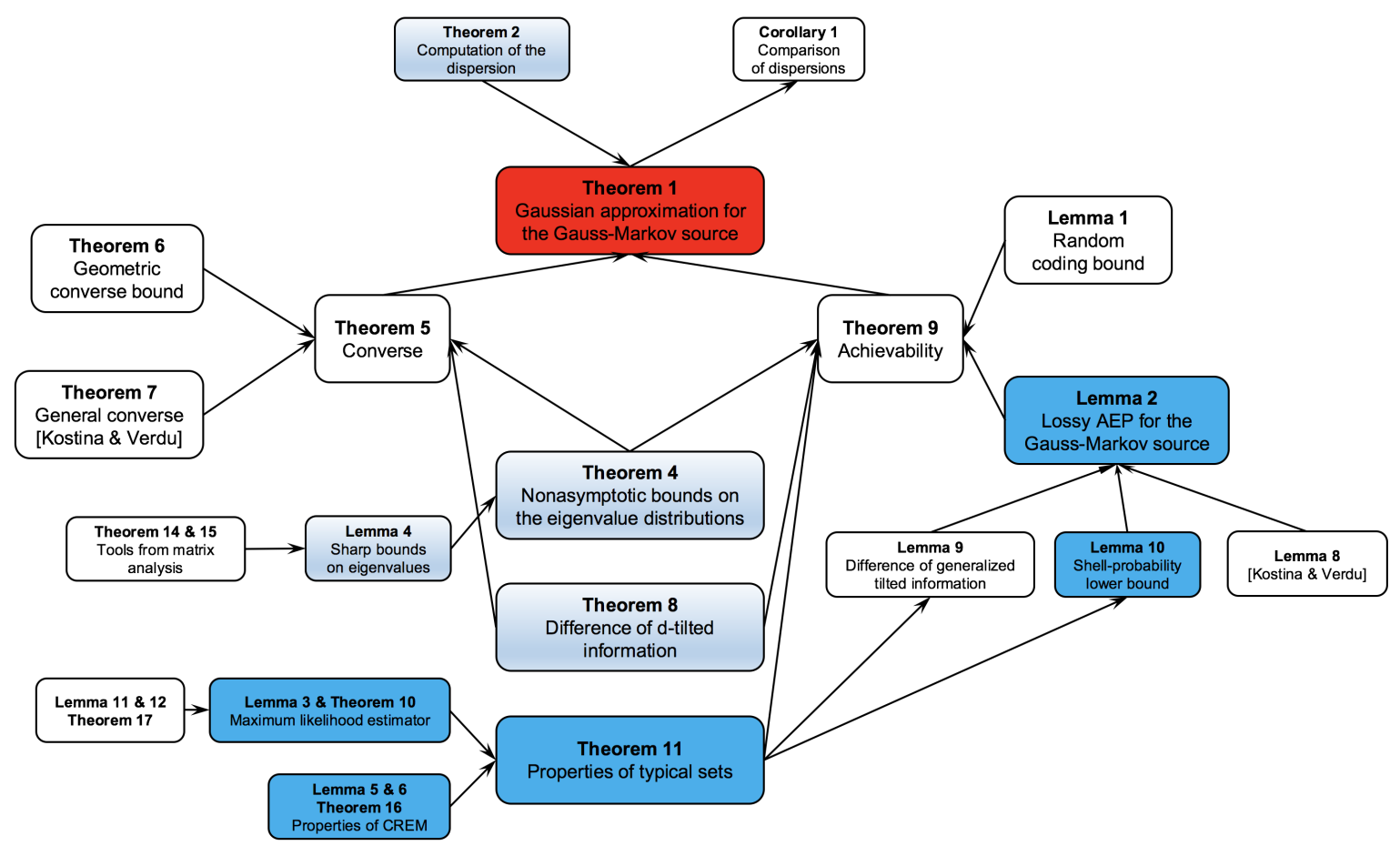

Fig. 4: A roadmap of the paper: an arrow from block $A$ to block $B$ means that proof or derivation of block $B$ is based on block $A$. Our main result is Theorem 1. The results in Theorem 2, Theorem 4, Theorem 8, and Lemma 4 are novel but not very hard to derive. The hardest part of the proof consists of Theorem 11. Lemma 10, Lemma 2 and their proofs.

\section{Corner points on the dispersion curve}

We derive (35) using the residue theorem from complex analysis, e.g. [35, p. 150, Th. 17]. Similar ideas have been applied by Berger [10, Chap. 6, p. 232] and Gray [26, Eq. (12)] to study the rate-distortion functions for nonstationary Gaussian autoregressive processes (19). The coordinate of $P_{1}$ can be easily obtained as follows. The water level $\theta_{c}>0$ matched to $d_{c}$ via 22 is

$$
\theta_{c}=\min _{w \in[-\pi, \pi]} \frac{\sigma^{2}}{g(w)},
$$

where the function $g$ is defined in (24). Therefore, (33) is simplified as

$$
V\left(d_{c}\right)=\frac{1}{4 \pi} \int_{-\pi}^{\pi} 1 d w=\frac{1}{2} .
$$

To treat $P_{2}$, note that the water level $\theta_{\max }>0$ matched to $d_{\max }$ via 22 is

$$
\theta_{\max }=\max _{w \in[-\pi, \pi]} \frac{\sigma^{2}}{g(w)}=\frac{\sigma^{2}}{(1-a)^{2}},
$$

which implies that

$$
V\left(d_{\max }\right)=\frac{\sigma^{4}}{4 \pi \theta_{\max }^{2}} \int_{-\pi}^{\pi} \frac{1}{(g(w))^{2}} d w .
$$

Invoking the residue theorem, we will obtain the integral

$$
\mathcal{I} \triangleq \int_{-\pi}^{\pi} \frac{1}{(g(w))^{2}} d w=\frac{2 \pi\left(1+a^{2}\right)}{\left(1-a^{2}\right)^{3}},
$$

which will complete the derivation. To that end, change variables using $z=e^{\mathrm{j} w}$ and rewrite

$$
\begin{aligned}
g(w) & =1+a^{2}-a\left(z+z^{-1}\right) \\
& =\left(z^{-1}-a\right)(z-a) .
\end{aligned}
$$

The integral $\mathcal{I}$ is then

$$
\begin{aligned}
\mathcal{I} & =\oint_{|z|=1} \frac{z}{\mathrm{j} a^{2}\left(z-a^{-1}\right)^{2}(z-a)^{2}} d z \\
& =2 \pi \mathrm{j} \operatorname{Res}_{z=a} \frac{z}{\mathrm{j} a^{2}\left(z-a^{-1}\right)^{2}(z-a)^{2}} \\
& =\frac{2 \pi}{a^{2}} \lim _{z \rightarrow a} \frac{d}{d z} \frac{z}{\left(z-a^{-1}\right)^{2}} \\
& =\frac{2 \pi\left(1+a^{2}\right)}{\left(1-a^{2}\right)^{3}},
\end{aligned}
$$

where (163) is by the change of variable $z=e^{\mathrm{j} w}$, and (164) is due to the residue theorem and $0 \leq a<1$, and (165) is the standard method of computing residue.

\section{Two interpretations of $d_{\max }$}

We present the details on computation of 27) leading to 31, and computation of the stationary variance in 29). These 
computations verify $(29)$. Using the same technique as in (163)(166), we compute (27) as

$$
\begin{aligned}
d_{\max } & =\frac{\sigma^{2}}{2 \pi} \oint_{|z|=1} \frac{1}{\mathrm{j} z\left(z^{-1}-a\right)(z-a)} d z \\
& =\sigma^{2} \operatorname{Res}_{z=a} \frac{1}{-a\left(z-a^{-1}\right)(z-a)} \\
& =\sigma^{2} \lim _{z \rightarrow a} \frac{1}{-a\left(z-a^{-1}\right)} \\
& =\frac{\sigma^{2}}{1-a^{2}} .
\end{aligned}
$$

To compute the stationary variance, take the variance on both sides of (2)

$$
\operatorname{Var}\left[U_{i}\right]=a^{2} \operatorname{Var}\left[U_{i-1}\right]+\sigma^{2},
$$

then take the limit on both sides of 171

$$
\lim _{i \rightarrow \infty} \operatorname{Var}\left[U_{i}\right]=a^{2} \lim _{i \rightarrow \infty} \operatorname{Var}\left[U_{i-1}\right]+\sigma^{2},
$$

which implies

$$
\lim _{i \rightarrow \infty} \operatorname{Var}\left[U_{i}\right]=\frac{\sigma^{2}}{1-a^{2}} .
$$

\section{APPENDIX B}

\section{PROOFS IN SECTION}

\section{A. Eigenvalues of $\mathrm{A}^{T} \mathrm{~A}$}

A sharp bound on the eigenvalues of $A^{T} A$, stated in Lemma 4 below, is key to proving Theorem 4 Its proof leverages the following tools of matrix theory, stated next in Theorem 14 and Theorem 15

Theorem 14 (Cauchy Interlacing Theorem for eigenvalues [36 p.59]). Let $\mathrm{H}$ be an $n \times n$ Hermitian matrix partitioned as $\mathrm{H}=$ $\left(\begin{array}{cc}\mathrm{P} & \star \\ \star & \star\end{array}\right)$, where $\mathrm{P}$ is an $(n-1) \times(n-1)$ principal submatrix of $\mathrm{H}$. Let $\lambda_{1}(\mathrm{P}) \leq \lambda_{2}(\mathrm{P}) \ldots \leq \lambda_{n-1}(\mathrm{P})$ be eigenvalues of $\mathrm{P}$, and $\lambda_{1}(\mathrm{H}) \leq \lambda_{2}(\mathrm{H}) \ldots \leq \lambda_{n}(\mathrm{H})$ be eigenvalues of $\mathrm{H}$, then $\lambda_{i}(\mathrm{H}) \leq \lambda_{i}(\mathrm{P}) \leq \lambda_{i+1}(\mathrm{H})$ for $i=1, \ldots, n-1$.

Theorem 15 (Gershgorin circle theorem, [37, p.16, Th. 1.11]). Let $\mathrm{M}$ be any $n \times n$ matrix, with entries $m_{i j}$. Define

$$
r_{i} \triangleq \sum_{j \neq i}\left|m_{i j}\right|,
$$

then for any eigenvalue $\lambda$ of $\mathrm{M}$, there exists $i \in[n]$ such that

$$
\left|\lambda-m_{i i}\right| \leq r_{i} .
$$

Lemma 4 (Sharp approximation of the eigenvalues of $A^{T} A$ ). For any $n \in \mathbb{N}$, let $\mu_{1} \leq \mu_{2} \ldots \leq \mu_{n}$ be the eigenvalues of $\mathrm{A}^{T} \mathrm{~A}$, and let

$$
\xi_{i}=g\left(\frac{i \pi}{n+1}\right)=1+a^{2}-2 a \cos \left(\frac{i \pi}{n+1}\right), \quad \forall i \in[n],
$$

where $g$ is defined in (24). Then, we have

$$
0 \leq \xi_{i}-\mu_{i} \leq \frac{2 a \pi}{n}, \quad \forall i \in[n] .
$$

Proof. To indicate the dimension, denote by $\mathrm{A}_{n}$ the matrix $\mathrm{A}$ defined in 39), and denote

$$
\begin{aligned}
\mathrm{B}_{n} \triangleq \mathrm{A}_{n}^{T} \mathrm{~A}_{n} & \\
& =\left(\begin{array}{cccccc}
1+a^{2} & -a & 0 & 0 & \ldots & 0 \\
-a & 1+a^{2} & -a & 0 & \ldots & 0 \\
0 & -a & 1+a^{2} & -a & \ddots & \vdots \\
\vdots & \ddots & \ddots & \ddots & \ddots & \vdots \\
\vdots & \ddots & 0 & -a & 1+a^{2} & -a \\
0 & \ldots & \ldots & 0 & -a & 1
\end{array}\right) .
\end{aligned}
$$

Notice that we obtain a tridiagonal Toeplitz matrix $\mathrm{W}_{n}$ if we replace the $(n, n)$-th entry of $\mathrm{B}_{n}$ by $1+a^{2}$ :

$$
\mathrm{W}_{n}=\left(\begin{array}{cccccc}
1+a^{2} & -a & 0 & 0 & \ldots & 0 \\
-a & 1+a^{2} & -a & 0 & \cdots & 0 \\
0 & -a & 1+a^{2} & -a & \ddots & \vdots \\
\vdots & \ddots & \ddots & \ddots & \ddots & \vdots \\
\vdots & \ddots & 0 & -a & 1+a^{2} & -a \\
0 & \ldots & \ldots & 0 & -a & 1+a^{2}
\end{array}\right) .
$$

Explicit formulas for eigenvalues of tridiagonal Toeplitz matrices are well-known, e.g. [38]. Namely, let $\xi_{1}^{(n)} \leq \xi_{2}^{(n)} \ldots \leq$ $\xi_{n}^{(n)}$ be eigenvalues of $\mathrm{W}_{n}$ listed in increasing order, then $\xi_{i}^{(n)}, \forall i \in[n]$ are given by (176). At an intuitive level, we expect $\xi_{i}^{(n)}$,s to approximate $\mu_{i}$ 's well since $\mathrm{B}_{n}$ and $\mathrm{W}_{n}$ differ in only one entry.

The first part of the proof applies Cauchy interlacing theorem (Theorem 14 to show (177) for $2 \leq i \leq n$. The bound (177) for $i=1$ is proved via Gershgorin circle theorem (Theorem [15) in the second part.

Applying the Theorem 14 by partitioning $\mathrm{B}_{n}$ as

$$
\mathrm{B}_{n}=\left(\begin{array}{cc}
\mathrm{W}_{n-1} & \star \\
\star & 1
\end{array}\right)
$$

we obtain

$$
\mu_{i} \leq \xi_{i}^{(n-1)} \leq \mu_{i+1}, \quad \forall i \in[n-1] .
$$

On the other hand, since $\mathrm{W}_{n} \succeq \mathrm{B}_{n}$ in semidefinite order, we have

$$
\xi_{i}^{(n)} \geq \mu_{i}, \quad \forall i \in[n] .
$$

Combining (181) and (182) yields

$$
\xi_{i-1}^{(n-1)} \leq \mu_{i} \leq \xi_{i}^{(n)}, \quad \forall i=2, \ldots, n .
$$

Simple algebraic manipulations using the formula (176) for the eigenvalues $\xi_{i}$ of $\mathrm{W}_{n}$ and (183) lead to

$$
\xi_{i}^{(n)}-\mu_{i} \leq \xi_{i}^{(n)}-\xi_{i-1}^{(n-1)} \leq \frac{2 \pi a}{n}, \quad \forall i=2, \ldots, n .
$$

To bound the difference $\xi_{1}^{(n)}-\mu_{1}$, we apply Gershgorin circle theorem (Theorem 15) to $\mathrm{B}_{n}$, defined in (178). Note that $r_{1}=r_{n}=a$ and $r_{i}=2 a, \forall i=2, \ldots, n-1$. For the 
eigenvalue $\mu_{1}$, there exists $j \in[n]$ such that $\left|\mu_{1}-\mathrm{B}_{j j}\right| \leq r_{j}$. The following analyses show that

$$
\mu_{1} \geq(1-a)^{2} .
$$

- If $2 \leq j \leq n-1$, then $\left|\mu_{1}-\left(1+a^{2}\right)\right| \leq 2 a$, which implies that $\mu_{1} \geq 1+a^{2}-2 a$.

- If $j=1$, then $\left|\mu_{1}-\left(1+a^{2}\right)\right| \leq a$, which implies that $\mu_{1} \geq 1+a^{2}-a \geq 1+a^{2}-2 a$.

- If $j=n$, then $\left|\mu_{1}-1\right| \leq a$, which implies $\mu_{1} \geq 1-a \geq$ $(1-a)^{2}$.

Recall from 176 that $\xi_{1}^{(n)}=1+a^{2}-2 a \cos \left(\frac{\pi}{n+1}\right)$. Hence,

$$
\begin{aligned}
\xi_{1}^{(n)}-\mu_{1} & \leq 2 a\left[1-\cos \left(\frac{\pi}{n+1}\right)\right] \\
& \leq \frac{a \pi^{2}}{(n+1)^{2}}, \\
& \leq \frac{2 a \pi}{n}
\end{aligned}
$$

where 187 is by the inequality $\cos (x) \geq 1-x^{2} / 2$. The validity of (177) for $i=1$ follows.

Remark 4. Lemma 4 and its proof imply immediately that

$$
(1-a)^{2} \leq \mu_{1} \leq \mu_{i} \leq(1+a)^{2},
$$

for any $i \in[n]$ and any $n \in \mathbb{N}$. That is, for any $n \in \mathbb{N}$, the eigenvalues of $A A^{T}$ are bounded by positive constants from both below and above.

\section{B. Proof of Theorem 4}

The proof relies on the sharp bound (Lemma 4p on the eigenvalues of $\mathrm{A}^{T} \mathrm{~A}$. Note that the function $\frac{\sigma^{2}}{g(w)}$ is even on $w \in[-\pi, \pi]$, hence

$$
\begin{aligned}
I & \triangleq \frac{1}{2 \pi} \int_{-\pi}^{\pi} F\left[\frac{\sigma^{2}}{g(w)}\right] d w \\
& =\frac{1}{\pi} \int_{0}^{\pi} F\left[\frac{\sigma^{2}}{g(w)}\right] d w .
\end{aligned}
$$

We will bound the integral $I$ by Riemann sums over intervals of width $\frac{\pi}{n+1}$, see Fig 5 Since $F\left[\frac{\sigma^{2}}{g(w)}\right]$ is a non-increasing function in $w \in[0, \pi]$, we have

$$
I \geq \frac{1}{\pi} \sum_{i=1}^{n} F\left[\frac{\sigma^{2}}{g\left(\frac{i \pi}{n+1}\right)}\right] \frac{\pi}{n+1} .
$$

Applying Lemma 4 to the argument of $F$ in 192 yields

$$
I \geq \frac{1}{n+1} \sum_{i=1}^{n} F\left(\frac{\sigma^{2}}{\mu_{i}+2 a \pi / n}\right) .
$$

Since $F$ is $L$-Lipschitz, for any $i \in[n]$, we have

$$
\begin{aligned}
F\left(\frac{\sigma^{2}}{\mu_{i}+2 a \pi / n}\right) & \geq F\left(\frac{\sigma^{2}}{\mu_{i}}\right)-L\left(\frac{\sigma^{2}}{\mu_{i}}-\frac{\sigma^{2}}{\mu_{i}+2 a \pi / n}\right) \\
& \geq F\left(\frac{\sigma^{2}}{\mu_{i}}\right)-\frac{2 a \pi L \sigma^{2}}{n \mu_{i}^{2}} .
\end{aligned}
$$

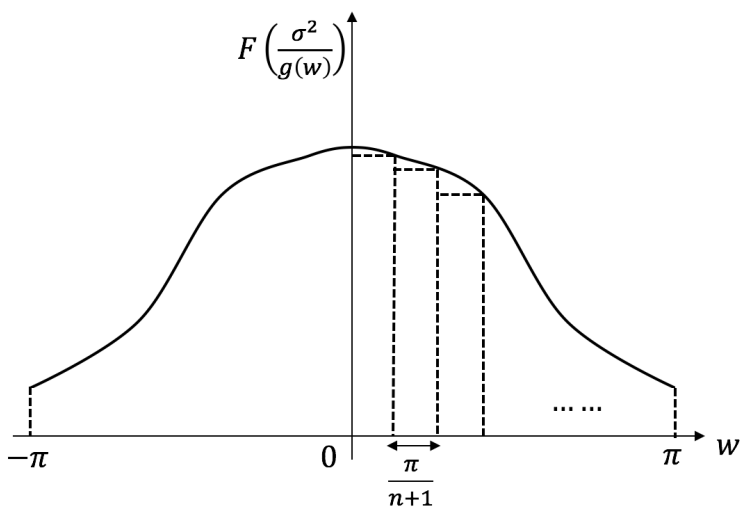

Fig. 5: Bound the integral $I$ by Riemann sum.

Plugging (195) into (193), we obtain

$$
I \geq \frac{1}{n+1} \sum_{i=1}^{n} F\left(\frac{\sigma^{2}}{\mu_{i}}\right)-\frac{2 a L \pi \sigma^{2}}{n(n+1)} \sum_{i=1}^{n} \frac{1}{\mu_{i}^{2}} .
$$

The bound 189 implies that

$$
\frac{1}{n} \sum_{i=1}^{n} \frac{1}{\mu_{i}^{2}} \leq \frac{1}{(1-a)^{4}}, \quad \forall n \in \mathbb{N} .
$$

Let $\max |F|$ be the maximum absolute value of $F$ over the working interval (46), then we have

$$
\begin{aligned}
I & \geq \frac{1}{n+1} \sum_{i=1}^{n} F\left(\frac{\sigma^{2}}{\mu_{i}}\right)-\frac{2 a L \pi \sigma^{2}}{(n+1)(1-a)^{4}} \\
& \geq \frac{1}{n} \sum_{i=1}^{n} F\left(\frac{\sigma^{2}}{\mu_{i}}\right)-\frac{\max |F|+2 a L \pi \sigma^{2} /(1-a)^{4}}{n} .
\end{aligned}
$$

In the same manner, we can derive the upper bound

$$
I \leq \frac{1}{n} \sum_{i=1}^{n} F\left(\frac{\sigma^{2}}{\mu_{i}}\right)+\frac{2 \max |F|}{n} .
$$

Therefore, setting

$$
C_{L} \triangleq \max \left\{\max |F|+\frac{2 a L \pi \sigma^{2}}{(1-a)^{4}}, 2 \max |F|\right\}
$$

completes the proof.

\section{Properties of conditional relative entropy minimization}

1) Gaussian CREM: In the optimization problem (49), if $\mathbf{X}$ and $\mathbf{Y}$ are Gaussian random vectors with independent coordinates, then we refer to (49) as Gaussian CREM.

Lemma 5. Let $\mathbf{X}$ and $\mathbf{Y}$ be Gaussian random vectors with independent coordinates, i.e.,

$$
\begin{aligned}
& \mathbf{X} \sim \mathcal{N}\left(\mathbf{0}, \Sigma_{\mathbf{X}}\right), \quad \text { where } \Sigma_{\mathbf{X}}=\operatorname{diag}\left(\alpha_{1}^{2}, \ldots, \alpha_{n}^{2}\right), \\
& \mathbf{Y} \sim \mathcal{N}\left(\mathbf{0}, \Sigma_{\mathbf{Y}}\right), \quad \text { where } \Sigma_{\mathbf{Y}}=\operatorname{diag}\left(\beta_{1}^{2}, \ldots, \beta_{n}^{2}\right),
\end{aligned}
$$

then the optimizer $P_{\mathbf{F}^{\star} \mid \mathbf{X}}$ in Gaussian CREM (49) with $\mathbf{X}$ and $\mathbf{Y}$ in 202, 203) is

$$
P_{\mathbf{F}^{\star} \mid \mathbf{X}}=\prod_{i=1}^{n} P_{F_{i}^{\star} \mid X_{i}},
$$


where for all $\mathbf{x} \in \mathbb{R}^{n}$, the conditional distribution of $F_{i}^{\star}$ given $X_{i}=x_{i}$ i ${ }^{5}$

$$
F_{i}^{\star} \mid\left\{X_{i}=x_{i}\right\} \sim \mathcal{N}\left(\frac{2 \lambda^{\star} \beta_{i}^{2} x_{i}}{1+2 \lambda^{\star} \beta_{i}^{2}}, \frac{\beta_{i}^{2}}{1+2 \lambda^{\star} \beta_{i}^{2}}\right),
$$

and the optimal value of Gaussian CREM (49) is given by

$$
\begin{aligned}
& \mathbb{R}(\mathbf{X}, \mathbf{Y}, d)= \\
& -\lambda^{\star} d+\frac{1}{2 n} \sum_{i=1}^{n} \log \left(1+2 \lambda^{\star} \beta_{i}^{2}\right)+\frac{1}{n} \sum_{i=1}^{n} \frac{\lambda^{\star} \alpha_{i}^{2}}{1+2 \lambda^{\star} \beta_{i}^{2}},
\end{aligned}
$$

where $\lambda^{\star}$ is the negative slope defined in 52 .

Proof. We particularize (51) to the Gaussian CREM. We first prove (204) and 205]. For any fixed $\mathrm{x} \in \mathbb{R}^{n}$, rearranging (51) yields

$$
\begin{aligned}
& f_{\mathbf{F}^{\star} \mid \mathbf{X}}(\mathbf{y} \mid \mathbf{x}) \\
= & f_{\mathbf{Y}}(\mathbf{y}) \exp \left\{\Lambda_{\mathbf{Y}}\left(\mathbf{x}, \lambda^{\star}, d\right)-\lambda^{\star} n \mathbf{d}(\mathbf{x}, \mathbf{y})+\lambda^{\star} n d\right\} \\
& \propto f_{\mathbf{Y}}(\mathbf{y}) \exp \left\{-\lambda^{\star} n \mathbf{d}(\mathbf{x}, \mathbf{y})\right\} \\
& \propto \exp \left\{-\lambda^{\star} \sum_{i=1}^{n}\left(y_{i}-x_{i}\right)^{2}-\sum_{i=1}^{n} \frac{y_{i}^{2}}{2 \beta_{i}^{2}}\right\} \\
= & \prod_{i=1}^{n} \exp \left\{-\frac{\left(y_{i}-\frac{2 \lambda^{\star} \beta_{i}^{2} x_{i}}{1+2 \lambda^{\star} \beta_{i}^{2}}\right)^{2}}{\frac{2 \beta_{i}^{2}}{1+2 \lambda^{\star} \beta_{i}^{2}}}\right\},
\end{aligned}
$$

where $p_{1} \propto p_{2}$ means that $p_{1}=c^{\prime} p_{2}$ for a positive constant $c^{\prime}$; (208) is by keeping only terms containing $\mathbf{y}$ (since $\mathbf{x}$ is fixed); (209) is by plugging the pdf of $\mathbf{Y}$ into (208); and (210) is by completing the squares in $y_{i}$. Hence, (204) and (205) are proved.

The expression 206 for the optimal value of the Gaussian CREM is obtained by direct computations using (51), 204 and (205).

$$
\begin{aligned}
& \mathbb{R}(\mathbf{X}, \mathbf{Y}, d) \\
= & \frac{1}{n} \int_{\mathbb{R}^{n}} f_{\mathbf{X}}(\mathbf{x}) \int_{\mathbb{R}^{n}} f_{\mathbf{F}^{\star} \mid \mathbf{X}}(\mathbf{y} \mid \mathbf{x})\left[\Lambda_{\mathbf{Y}}\left(\mathbf{x}, \lambda^{\star}, d\right)-\right. \\
= & \frac{1}{n} \int_{\mathbb{R}^{n}} f_{\mathbf{X}}(\mathbf{x}) \int_{\mathbb{R}^{n}} f_{\mathbf{F}^{\star} \mid \mathbf{X}}(\mathbf{y} \mid \mathbf{x}) \Lambda_{\mathbf{Y}}\left(\mathbf{x}, \lambda^{\star}, d\right) d \mathbf{y} d \mathbf{x} \\
= & -\lambda^{\star} d+\frac{1}{2 n} \sum_{i=1}^{n} \log \left(1+2 \lambda^{\star} \beta_{i}^{2}\right)+\frac{1}{n} \sum_{i=1}^{n} \frac{\lambda^{\star} \alpha_{i}^{2}}{1+2 \lambda^{\star} \beta_{i}^{2}},
\end{aligned}
$$

where (211) follows by substituting (51) into 49 ; 212 ) holds since $\mathbb{E}\left[\mathrm{d}\left(\mathbf{X}, \mathbf{F}^{\star}\right)\right]=d$ by the optimality of $\mathbf{F}^{\star}$; and (213) is by direct integration of 212, which relies on the definition of generalized tilted information (53), gaussianity of $\mathbf{X}$ and $\mathbf{Y}$, and (204).

\footnotetext{
${ }^{5}$ When $\beta_{i}^{2}=0$ for some $i \in[n]$, the random variable in 205 degenerates to a deterministic random variable taking value 0 , and the notation $\mathcal{N}(0,0)$ denotes the Dirac delta function.
}

2) Gauss-Markov CREM: In the optimization problem (49), if $\mathbf{X}$ is the decorrelation of $\mathbf{U}$, i.e., $\mathbf{X}$ is given by (41), and $(\mathbf{X}, \mathbf{Y})=\left(\mathbf{X}, \mathbf{Y}^{\star}\right)$ forms a RDF-achieving pair, then we refer (49) as Gauss-Markov CREM. Recall from (44) that $\mathbf{X} \sim \mathcal{N}\left(0, \Sigma_{\mathbf{X}}\right)$, where

$$
\Sigma_{\mathbf{X}}=\operatorname{diag}\left(\sigma_{1}^{2}, \ldots, \sigma_{n}^{2}\right),
$$

and $\sigma_{i}^{2}, \forall i \in[n]$ is given by [45]. From the reverse waterfilling solution for the parallel Gaussian source [9. Th. 10.3.3], we know that $\mathbf{Y}^{\star} \sim \mathcal{N}\left(0, \Sigma_{\mathbf{Y}^{\star}}\right)$, where

$$
\Sigma_{\mathbf{Y}^{\star}}=\operatorname{diag}\left(\nu_{1}^{2}, \ldots, \nu_{n}^{2}\right),
$$

and for any $i \in[n]$,

$$
\begin{aligned}
& \nu_{i}^{2} \triangleq \sigma_{i}^{2}-d_{i}, \\
& d_{i} \triangleq \min \left(\theta_{n}, \sigma_{i}^{2}\right),
\end{aligned}
$$

where $\theta_{n}>0$ is the water level matched to $d$ via the $n$-th order reverse waterfilling [15]. It is known [29], [30] that

$$
\mathbb{R}\left(\mathbf{X}, \mathbf{Y}^{\star}, d\right)=\mathbb{R}_{\mathbf{U}}(n, d),
$$

where $\mathbb{R}_{\mathbf{U}}(n, d)$ is given by (14).

Lemma 6. In Gauss-Markov CREM, for any $d \in\left(0, d_{\max }\right)$ and $n \in \mathbb{N}$, let $\theta_{n}>0$ be the water level matched to $d$ via the $n$-th order reverse waterfilling (15), the negative slope $\lambda^{\star \star}$ defined in (9) satisfies

$$
\lambda^{\star \star}=\frac{1}{2 \theta_{n}} .
$$

Proof. We directly compute the negative slope using the parametric representation (14) and (15). Taking the derivative with respect to $d$ on both sides of (14) yields

$$
\lambda^{\star \star}=\frac{1}{n} \sum_{i=1}^{n} \frac{1}{2 \theta_{n}} \frac{d \theta_{n}}{d d} \mathbb{1}\left\{\sigma_{i}^{2}>\theta_{n}\right\} .
$$

Differentiating (15), we obtain

$$
\frac{d d}{d \theta_{n}}=\frac{1}{n} \sum_{i=1}^{n} \mathbb{1}\left\{\sigma_{i}^{2}>\theta_{n}\right\},
$$

which is independent of $i$. Plugging 221 into 220 yields 219.

To justify the formal differentiation in 221, observe using (15) that $d$ is a continuous piecewise linear function of $\theta_{n}$, and $d$ is differentiable with respect to $\theta_{n}$ except when $\theta_{n}=\sigma_{i}^{2}, i \in[n]$. The above proof will go through as long as the derivatives at those $n$ points are understood to be the left derivatives. Indeed, $\mathbb{R}_{\mathbf{U}}(n, d)$ is differentiable w.r.t. $d$ for any $d \in\left(0, d_{\max }\right)$, e.g. [13, Eq. (16)].

3) Sensitivity of Gauss-Markov CREM: The following result is needed in our achievability proof (the proof of Theorem 9).

Theorem 16. Let $\mathbf{X}$ be the decorrelation of $\mathbf{U}$, defined in (41), and let $\left(\mathbf{X}, \mathbf{Y}^{\star}\right)$ be a RDF-achieving pair. For any fixed distortion $d \in\left(0, d_{\max }\right)$, let $\theta>0$ be the water level matched 
to $d$ via the limiting reverse waterfilling in (22). For any $\kappa \in(0, \theta / 3)$, let $\hat{\sigma}_{i}^{2}, i \in[n]$ be such that

$$
\left|\hat{\sigma}_{i}^{2}-\sigma_{i}^{2}\right| \leq \kappa, \forall i \in[n] .
$$

Define the Gaussian random vector $\hat{\mathbf{X}} \sim$ $\mathcal{N}\left(\mathbf{0}, \operatorname{diag}\left(\hat{\sigma}_{1}^{2}, \ldots, \hat{\sigma}_{n}^{2}\right)\right)$, and let $\hat{\lambda}^{\star}$ be the negative slope of $\mathbb{R}\left(\hat{\mathbf{X}}, \mathbf{Y}^{\star}, d\right)$. Then, there exists $N(d) \in \mathbb{N}$ such that for all $n \geq N(d)$, the negative slope $\hat{\lambda}^{\star}$ satisfies

$$
\left|\lambda^{\star \star}-\hat{\lambda}^{\star}\right| \leq \frac{9 \kappa}{4 \theta^{2}},
$$

where $\lambda^{\star \star}$ is defined in 9 .

Proof. Consider the Gaussian CREM $\mathbb{R}\left(\hat{\mathbf{X}}, \mathbf{Y}^{\star}, d\right)$, where $\mathbf{Y}^{\star}$, given by 215), is such that $\left(\mathbf{X}, \mathbf{Y}^{\star}\right)$ forms a RDF-achieving pair. Let $\theta_{n}>0$ be the water level matched to $d$ via the $n$-th order reverse waterfilling $\left[15\right.$ over $\hat{\sigma}_{i}^{2}, i \in[n]$, and let $\theta_{n}>0$ be the water level matched to $d$ via the $n$-th order reverse waterfilling $(15)$ over $\sigma_{i}^{2}, i \in[n]$. In (206), replacing $\mathbf{X}, \mathbf{Y}$ by $\hat{\mathbf{X}}, \mathbf{Y}^{\star}$, and then taking the derivative with respect to $d$ on both sides yields

$$
\begin{aligned}
-\hat{\lambda}^{\star} \triangleq & \frac{d \mathbb{R}\left(\hat{\mathbf{X}}, \mathbf{Y}^{\star}, d\right)}{d d} \\
= & -\hat{\lambda}^{\star}+\frac{1}{2 n} \sum_{i: \hat{\sigma}_{i}^{2}>\hat{\theta}_{n}} \frac{-2 \hat{\lambda}^{\star}}{1+2 \hat{\lambda}^{\star} \nu_{i}^{2}} \frac{d \hat{\theta}_{n}}{d d}+ \\
& \frac{1}{n} \sum_{i: \hat{\sigma}_{i}^{2}>\hat{\theta}_{n}} \frac{2 \hat{\sigma}_{i}^{2} \hat{\lambda}^{\star 2}}{\left(1+2 \hat{\lambda}^{\star} \nu_{i}^{2}\right)^{2}} \frac{d \hat{\theta}_{n}}{d d} .
\end{aligned}
$$

Rearranging terms yields

$$
\hat{\lambda}^{\star}=\frac{\sum_{i: \hat{\sigma}_{i}^{2}>\hat{\theta}_{n}} \frac{1}{\left(1+2 \hat{\lambda}^{\star} \nu_{i}^{2}\right)^{2}}}{\sum_{i: \hat{\sigma}_{i}^{2}>\hat{\theta}_{n}} \frac{2\left(\hat{\sigma}_{i}^{2}-\sigma_{i}^{2}+\theta_{n}\right)}{\left(1+2 \hat{\lambda}^{\star} \nu_{i}^{2}\right)^{2}}},
$$

where the relation 216 is used. Substituting the bounds 222 into 226, we obtain

$$
\hat{\lambda}^{\star} \in\left[\frac{1}{2\left(\theta_{n}+\kappa\right)}, \frac{1}{2\left(\theta_{n}-\kappa\right)}\right] .
$$

As shown in Appendix $\mathrm{C}-\mathrm{A}$ below, there exists a constant $C_{\theta}>0$ (depending on $\theta$, or equivalently $d$ ) such that for all large $n$,

$$
\left|\theta-\theta_{n}\right| \leq \frac{\mathrm{C}_{\theta}}{n} .
$$

Choosing $N(d) \geq 3 \mathrm{C}_{\theta} / \theta$, we have for all $n \geq N(d)$,

$$
\frac{2 \theta}{3} \leq \theta_{n} \leq \frac{4 \theta}{3} \text {. }
$$

Since by the assumption, $0<\kappa<\theta / 3$, 229) implies that $0<\kappa<\theta_{n} / 2$. Recalling 219, we have from (227) and 229)

$$
\begin{aligned}
& \left|\lambda^{\star \star}-\hat{\lambda}^{\star}\right| \\
\leq & \max \left\{\left|\frac{1}{2\left(\theta_{n}+\kappa\right)}-\frac{1}{2 \theta_{n}}\right|,\left|\frac{1}{2\left(\theta_{n}-\kappa\right)}-\frac{1}{2 \theta_{n}}\right|\right\} \\
\leq & \frac{\kappa}{\theta_{n}^{2}} \\
\leq & \frac{9 \kappa}{4 \theta^{2}} .
\end{aligned}
$$

\section{Proof of Theorem 2}

We need the following lemma.

Lemma 7 (Parametric representation for d-tilted information). Let $\mathbf{X}$ be the decorrelation of $\mathbf{U}(41)$, and let $\left(\mathbf{X}, \mathbf{Y}^{\star}\right)$ be a RDF-achieving pair. For any $d \in\left(0, d_{\max }\right)$, let $\theta_{n}>0$ be the water level matched to $d$ via the $n$-th order reverse waterfilling (15) over $\sigma_{i}^{2}, i \in[n]$, and let $d_{i} \triangleq \min \left(\theta_{n}, \sigma_{i}^{2}\right)$ be the target distortion for $X_{i}$. Then, for all $\mathbf{x} \in \mathbb{R}^{n}$,

$$
\begin{aligned}
\jmath \mathbf{X}(\mathbf{x}, d)= & \sum_{i=1}^{n} \Lambda_{Y_{i}^{\star}}\left(x_{i}, \lambda^{\star \star}, d_{i}\right), \\
\Lambda_{Y_{i}^{\star}}\left(x_{i}, \lambda^{\star \star}, d_{i}\right)= & \frac{\min \left(\theta_{n}, \sigma_{i}^{2}\right)}{2 \theta_{n}}\left(\frac{x_{i}^{2}}{\sigma_{i}^{2}}-1\right)+ \\
& \frac{1}{2} \log \frac{\max \left(\theta_{n}, \sigma_{i}^{2}\right)}{\theta_{n}}, \quad \forall i \in[n],
\end{aligned}
$$

where $\lambda^{\star \star}$ is defined in (9).

Proof. The relation 233) is by the independence of $Y_{1}^{\star}, \ldots, Y_{n}^{\star}$. Proof of (234) replies on the gaussianity of $\mathbf{Y}^{\star}$. For each $i \in[n]$, from (53) and 219], we have

$\Lambda_{Y_{i}^{\star}}\left(x_{i}, \lambda^{\star \star}, d_{i}\right)=-\frac{d_{i}}{2 \theta_{n}}-\log \mathbb{E}\left[\exp \left\{-\lambda^{\star \star}\left(Y_{i}^{\star}-x_{i}\right)^{2}\right\}\right]$.

Substituting $d_{i}=\sigma_{i}^{2}$ and $Y_{i}^{\star} \equiv 0$ a.s. for all $i$ s.t. $\sigma_{i}^{2} \leq \theta_{n}$ (recall 215) into 235, we obtain

$$
\Lambda_{Y_{i}^{\star}}\left(x_{i}, \lambda^{\star \star}, d_{i}\right)=\frac{x_{i}^{2}-\sigma_{i}^{2}}{2 \theta_{n}} .
$$

Substituting $d_{i}=\theta_{n}$ and $Y_{i}^{\star} \sim \mathcal{N}\left(0, \sigma_{i}^{2}-\theta_{n}\right)$ for those $i$ for which $\sigma_{i}^{2}>\theta_{n}$ (recall (215) into (235) and applying the formula for the moment generating function of non-central $\chi^{2}$-distribution with one degree of freedom, we compute

$$
\Lambda_{Y_{i}^{\star}}\left(x_{i}, \lambda^{\star \star}, d_{i}\right)=\frac{1}{2}\left(\frac{x_{i}^{2}}{\sigma_{i}^{2}}-1\right)+\frac{1}{2} \log \frac{\sigma_{i}^{2}}{\theta_{n}} .
$$

Unifying (236) and 237), we obtain 234).

Proof of Theorem 2 For any fixed distortion $d \in\left(0, d_{\max }\right)$, let $\theta>0$ be the water level matched to $d$ via the limiting reverse waterfilling (22). Taking the expectation and the variance of (233) using (234) yield 6

$$
\begin{aligned}
\mathbb{E}[\jmath \mathbf{X}(\mathbf{X}, d)] & =\sum_{i=1}^{n} \frac{1}{2} \max \left(0, \log \frac{\sigma_{i}^{2}}{\theta_{n}}\right), \\
\operatorname{Var}[\jmath \mathbf{X}(\mathbf{X}, d)] & =\sum_{i=1}^{n} \frac{1}{2} \min \left(1,\left(\frac{\sigma_{i}^{2}}{\theta_{n}}\right)^{2}\right) .
\end{aligned}
$$

An application of Theorem 3 to 238 with the function $F(t) \triangleq$ $\frac{1}{2} \max \left(0, \log \frac{t}{\theta}\right)$ yields 38). Similarly, an application of Theorem 3 to 239) with the function $F(t) \triangleq \frac{1}{2} \min \left[1,\left(\frac{t}{\theta}\right)^{2}\right]$ yields 377 .

\footnotetext{
${ }^{6}$ The result on expectations was established by Gray [4], which we recover here. The result on variances is new.
} 


\section{APPENDIX C \\ PROOFS IN SECTION III}

\section{A. Proof of Theorem 8}

The proof is based on Chebyshev's inequality. Fix $d \in$ $\left(0, d_{\text {max }}\right)$. For each fixed $n \in \mathbb{N}$, let $\theta_{1}, \theta_{2}>0$ be the water levels matched to $d$ and $d_{n}$, respectively, in the $n$-th order reverse waterfilling 15 over $\sigma_{i}^{2}, i \in[n]$, that is,

$$
\begin{aligned}
d & =\frac{1}{n} \sum_{i=1}^{n} \min \left(\theta_{1}, \sigma_{i}^{2}\right), \\
d_{n} & =\frac{1}{n} \sum_{i=1}^{n} \min \left(\theta_{2}, \sigma_{i}^{2}\right) .
\end{aligned}
$$

Of course, both $\theta_{1}$ and $\theta_{2}$ depend on $n$. Let $N_{1} \in \mathbb{N}$ be such that

$$
\left|d-d_{n}\right| \leq \frac{h_{1}}{n}, \quad \forall n \geq N_{1}
$$

We proceed to show that there exist constants $h_{2}>0$ and $N_{2} \in \mathbb{N}$ such that

$$
\left|\theta_{1}-\theta_{2}\right| \leq \frac{h_{2}}{n}, \quad \forall n \geq N_{2}
$$

Indeed, without loss of generality, assume $d<d_{n} 7$, then $\theta_{1}<\theta_{2}$. Define the following index sets

$$
\begin{aligned}
& I_{1} \triangleq\left\{i \in[n]: \sigma_{i}^{2} \leq \theta_{1}\right\}, \\
& I_{2} \triangleq\left\{i \in[n]: \theta_{1}<\sigma_{i}^{2}<\theta_{2}\right\}, \\
& I_{3} \triangleq\left\{i \in[n]: \theta_{2} \leq \sigma_{i}^{2}\right\} .
\end{aligned}
$$

Then,

$$
\begin{aligned}
d_{n}-d & =\frac{1}{n} \sum_{i=1}^{n}\left(\min \left(\theta_{2}, \sigma_{i}^{2}\right)-\min \left(\theta_{1}, \sigma_{i}^{2}\right)\right) \\
& =\frac{1}{n} \sum_{i \in I_{1}} 0+\frac{1}{n} \sum_{i \in I_{2}}\left(\sigma_{i}^{2}-\theta_{1}\right)+\frac{1}{n} \sum_{i \in I_{3}}\left(\theta_{2}-\theta_{1}\right) \\
& \geq \frac{\left|I_{3}\right|}{n}\left(\theta_{2}-\theta_{1}\right)
\end{aligned}
$$

Choose $N_{2} \in \mathbb{N}$ such that for all $n \geq N_{2},\left|I_{3}\right| \geq \tilde{c} n$ for some constant $\tilde{c} \in(0,1)$. This is possible because $d<d_{\max }$. Then 243 holds for $h_{2}=\frac{h_{1}}{\tilde{c}}$.

Now, let $G_{1}, \ldots, G_{n}$ be i.i.d. $\mathcal{N}(0,1)$. To simplify notation, denote the random variable

$$
\Delta\left(d, d_{n}\right)=\jmath \mathbf{X}(\mathbf{X}, d)-\jmath \mathbf{X}\left(\mathbf{X}, d_{n}\right) .
$$

Then from (233) and (234), we have

$$
\begin{aligned}
\Delta\left(d, d_{n}\right) & =\sum_{i=1}^{n}\left[\frac{\min \left\{\theta_{1}, \sigma_{i}^{2}\right\}}{2 \theta_{1}}-\frac{\min \left\{\theta_{2}, \sigma_{i}^{2}\right\}}{2 \theta_{2}}\right]\left(G_{i}^{2}-1\right) \\
& +\sum_{i=1}^{n} \frac{1}{2} \log \left[\frac{\max \left\{\theta_{1}, \sigma_{i}^{2}\right\}}{2 \theta_{1}} \cdot \frac{2 \theta_{2}}{\max \left\{\theta_{2}, \sigma_{i}^{2}\right\}}\right] .
\end{aligned}
$$

To apply Chebyshev's inequality, we bound the mean and variance of $\Delta\left(d, d_{n}\right)$ as follows.

$$
\begin{aligned}
\mathbb{E}\left[\Delta\left(d, d_{n}\right)\right] & =\sum_{i=1}^{n} \frac{1}{2} \log \left[\frac{\max \left\{\theta_{1}, \sigma_{i}^{2}\right\}}{2 \theta_{1}} \cdot \frac{2 \theta_{2}}{\max \left\{\theta_{2}, \sigma_{i}^{2}\right\}}\right] \\
& =\sum_{i \in I_{1}} 0+\sum_{i \in I_{2}} \frac{1}{2} \log \frac{\sigma_{i}^{2}}{\theta_{1}}+\sum_{i \in I_{3}} \frac{1}{2} \log \frac{\theta_{2}}{\theta_{1}} \\
& \leq \frac{h_{2}}{\theta_{1}}
\end{aligned}
$$

where 254) holds since for $i \in I_{2}$, we have $\frac{1}{2} \log \frac{\sigma_{i}^{2}}{\theta_{1}} \leq$ $\frac{1}{2} \log \frac{\theta_{2}}{\theta_{1}}$, while for $i \in I_{3}$, we have

$$
\frac{1}{2} \log \frac{\theta_{2}}{\theta_{1}} \leq \frac{1}{2} \log \left(1+\frac{h_{2}}{n \theta_{1}}\right) \leq \frac{h_{2}}{2 n \theta_{1}},
$$

where we used 243. By a similar argument, we can bound the variance as

$$
\operatorname{Var}\left[\Delta\left(d, d_{n}\right)\right] \leq \frac{h_{2}^{2}}{\theta_{1}^{2} n} .
$$

In conjunction with 254, 256, Chebyshev's inequality yields

$$
\mathbb{P}\left[\left|\Delta\left(d, d_{n}\right)-\mathbb{E}\left[\Delta\left(d, d_{n}\right)\right]\right| \geq \ell\right] \leq \frac{h_{2}^{2}}{\theta_{1}^{2} n \ell^{2}}, \quad \forall \ell>0 .
$$

Choosing $\ell=\frac{u h_{2}}{\theta_{1}}$ in 257 and applying 254 yields

$$
\mathbb{P}\left[\left|\Delta\left(d, d_{n}\right)\right| \geq \frac{(1+u) h_{2}}{\theta_{1}}\right] \leq \frac{1}{n u^{2}}, \quad \forall u>0 .
$$

Let $\theta>0$ be the water level matched to $d$ via the limiting reverse waterfilling (22), then by Theorem 4 and (240), there exists a constant $C_{L}>0$ such that

$$
\left|\theta_{1}-\theta\right| \leq \frac{C_{L}}{n}, \quad n \in \mathbb{N} .
$$

Choose $N_{3} \in \mathbb{N}$ such that $\theta_{1} \geq \frac{\theta}{2}, \forall n \geq N_{3}$. Then, for all $n \geq N_{3}$, we have

$$
\mathbb{P}\left[\left|\Delta\left(d, d_{n}\right)\right| \geq \frac{2(1+u) h_{2}}{\theta}\right] \leq \frac{1}{n u^{2}} .
$$

Taking $N(d) \triangleq \max \left(N_{1}, N_{2}, N_{3}\right)$ and rearranging the terms completes the proof.

\section{APPENDIX D \\ PROOFS IN SECTIONIV}

\section{A. Proof of Lemma 2}

In addition to new concentration inequalities (shown in Lemma 9 and Lemma 10 below), the proof leverages the following bound, which is a direct application of [13, Lem. 1] to the random vector $\mathbf{X}$.

Lemma 8 (Lower bound on probability of distortion balls). Fix $d \in\left(0, d_{\max }\right), n \in \mathbb{N}$, and distribution $P_{\mathbf{Y}}$ on $\mathbb{R}^{n}$. Then for any $\mathrm{x} \in \mathbb{R}^{n}$, it holds that

$$
\begin{array}{r}
P_{\mathbf{Y}}(\mathcal{B}(\mathbf{x}, d)) \geq \sup _{P_{\hat{\mathbf{x}}}, \gamma>0} \exp \left\{-\hat{\lambda}^{\star} n \gamma-\Lambda_{\mathbf{Y}}\left(\mathbf{x}, \hat{\lambda}^{\star}, d\right)\right\} \times \\
\quad \mathbb{P}\left[d-\gamma \leq \mathbf{d}\left(\mathbf{x}, \hat{\mathbf{F}}^{\star}\right) \leq d \mid \hat{\mathbf{X}}=\mathbf{x}\right],
\end{array}
$$

${ }^{7}$ Otherwise, switch $\theta_{1}$ and $\theta_{2}$ in the rest of the proof. 
where the supremum is over all pdfs $P_{\hat{\mathrm{X}}}$ on $\mathbb{R}^{n}$, and $\Lambda_{\mathbf{Y}}\left(\mathbf{x}, \hat{\lambda}^{\star}, d\right)$ is the generalized tilted information defined in (53), and

$$
\hat{\lambda}^{\star}=-\mathbb{R}^{\prime}(\hat{\mathbf{X}}, \mathbf{Y}, d),
$$

and the random variable $\hat{\mathbf{F}}^{\star}$ achieves $\mathbb{R}(\hat{\mathbf{X}}, \mathbf{Y}, d)$.

The high-level idea proving Lemma 2 is the following. In Lemma 8 choose $\mathbf{Y} \leftarrow \mathbf{Y}^{\star}$ defined in 215 , and choose $\hat{\mathbf{X}}$ to be the proxy Gaussian random variable with variances given by the ML estimates of the variances formally defined in (118) and (119). This choice of $\hat{\mathbf{X}}$ and $\mathbf{Y}$ enables us to further lower bound the two terms on the right-hand side of (261). The two lower bounds are presented in the next two lemmas.

The first bound is a concentration inequality on generalized tilted information.

Lemma 9. For any fixed $d \in\left(0, d_{\max }\right)$ and $\epsilon \in(0,1)$, there exists $N \in \mathbb{N}$, and constants $C, C_{2}>0$ such that for $n \geq N$,

$$
\begin{array}{r}
\mathbb{P}\left[\Lambda_{\mathbf{Y}^{\star}}\left(\mathbf{X}, \hat{\lambda}^{\star}(\mathbf{X}), d\right) \leq \Lambda_{\mathbf{Y}^{\star}}\left(\mathbf{X}, \lambda^{\star \star}, d\right)+C \log n\right] \\
\geq 1-\frac{C_{2}}{(\log n)^{c \alpha}},
\end{array}
$$

where $\hat{\lambda}^{\star}(\mathbf{x})$ is given by $(128)$ with $\hat{\mathbf{X}}$ being our proxy Gaussian random variable defined in (118) and (119), and $\lambda^{\star \star}$ is given by 9 .

Proof. Appendix D-C

The second bound, presented in Lemma 10 below, is referred to as shell-probability lower bound. For any $\mathbf{x} \in \mathbb{R}^{n}$ and any $\gamma \in(0, d)$, define the shell

$$
\mathcal{S}(\mathbf{x}, d, \gamma) \triangleq\left\{\mathbf{x}^{\prime} \in \mathbb{R}^{n}: d-\gamma \leq \mathrm{d}\left(\mathbf{x}, \mathbf{x}^{\prime}\right) \leq d\right\} .
$$

Geometrically, Lemma 8 provides a quantitative connection between the probability of a distortion ball and the probability of its shell. Lemma 10 below gives a lower bound on the probability of the shell $\mathcal{S}(\mathbf{x}, d, \gamma)$ for "typical" sequences $\mathbf{x}$.

Lemma 10 (Shell-probability lower bound). For any $\alpha>0$ and $n \in \mathbb{N}$, let $\mathcal{T}(n, \alpha, p)$ be defined in Definition 2 and $p$ be the constant in Theorem 11. For any $d \in\left(0, d_{\max }\right)$ and $\epsilon \in(0,1)$, let

$$
\gamma \triangleq \frac{\log ^{q} n}{n}
$$

where $q>1$ is a constant specified in the proof, then there exist constants $C_{1}>0$ and $N(d, \epsilon, \alpha) \in \mathbb{N}$ such that for any $n \geq N(d, \epsilon, \alpha)$, any $\mathbf{u} \in \mathcal{T}(n, \alpha, p)$, and $\mathbf{x}=\mathrm{S}^{T} \mathbf{u}$ with $\mathrm{S}$ in (43), it holds that

$$
\mathbb{P}\left[\hat{\mathbf{F}}^{\star} \in \mathcal{S}(\mathbf{x}, d, \gamma) \mid \hat{\mathbf{X}}=\mathbf{x}\right] \geq \frac{C_{1}}{\sqrt{n}},
$$

where $\hat{\mathbf{X}}$ is given in (119).

Proof. Appendix D-D.

Based on Lemma 8, Lemma 9 and Lemma 10 , we present the proof of Lemma 2
Proof of Lemma 2. Let $\mathbf{X}$ be the decorrelation of $\mathbf{U}$ in (41). Choose $\mathbf{Y} \leftarrow \mathbf{Y}^{\star}$ in Lemma 8 Let $\mathcal{T}(n, \alpha, p)$ be the set defined in Definition 2 and $p$ be the constant in Theorem 11 Let $C, C_{1}, C_{2}, q$ be the same constants in Lemma 9 and Lemma 10 Choose $N(d, \epsilon, \alpha) \in \mathbb{N}$ large enough such that Theorem 11 Lemma 9 and Lemma 10 hold. Let $\theta>0$ be the water level matched to $d$ via the limiting reverse waterfilling (22). Denote the event

$$
\mathcal{E} \triangleq\left\{\log \frac{1}{P_{\mathbf{Y}^{\star}}(\mathcal{B}(\mathbf{X}, d))}>\jmath \mathbf{X}(\mathbf{X}, d)+c_{1} \log ^{q} n+c_{2}\right\},
$$

where

$$
\begin{aligned}
& c_{1}=\frac{1}{2 \theta}+C_{d}+\frac{1}{2}+C, \\
& c_{2}=-\log C_{1},
\end{aligned}
$$

and $C_{d}>0$ is a constant such that

$$
\left|\hat{\lambda}^{\star}(\mathbf{x})-\frac{1}{2 \theta}\right| \leq C_{d}
$$

for any $\mathbf{u} \in \mathcal{T}(a, \alpha, p)$ and $\mathbf{x}=\mathrm{S}^{T} \mathbf{u}$. The existence of such $C_{d}$ is guaranteed by (127) in Theorem 11 and (243).

Using elementary probability rules, we write

$$
\mathbb{P}\left[\log \frac{1}{P_{\mathbf{Y}^{\star}}(\mathcal{B}(\mathbf{X}, d))}>\jmath_{\mathbf{X}}(\mathbf{X}, d)+c_{1} \log ^{q} n+c_{2}\right]
$$

$=\mathbb{P}[\mathcal{E}, \mathbf{U} \in \mathcal{T}(n, \alpha, p)]+\mathbb{P}[\mathcal{E}, \quad \mathbf{U} \notin \mathcal{T}(n, \alpha, p)]$

$$
\begin{aligned}
& \leq \mathbb{P}\left\{\mathbf{U} \in \mathcal{T}(n, \alpha, p), \hat{\lambda}^{\star}(\mathbf{X}) n \gamma+\Lambda_{\mathbf{Y}^{\star}}\left(\mathbf{X}, \hat{\lambda}^{\star}(\mathbf{X}), d\right)-\right. \\
& \quad \log \mathbb{P}\left[\hat{\mathbf{F}}^{\star} \in \mathcal{S}(\hat{\mathbf{X}}, d, \gamma) \mid \hat{\mathbf{X}}=\mathbf{X}\right]>\jmath \mathbf{X}(\mathbf{X}, d)+ \\
& \left.\quad c_{1} \log ^{q} n+c_{2}\right\} \\
& \quad+\mathbb{P}[\mathbf{U} \notin \mathcal{T}(n, \alpha, p)] \\
& \leq \mathbb{P}\left\{\mathbf{U} \in \mathcal{T}(n, \alpha, p), \Lambda_{\mathbf{Y}^{\star}}\left(\mathbf{X}, \hat{\lambda}^{\star}(\mathbf{X}), d\right)-\jmath \mathbf{X}(\mathbf{X}, d)>\right. \\
& \left.\quad-\hat{\lambda}^{\star}(\mathbf{X}) n \gamma+\log \frac{C_{1}}{\sqrt{n}}+c_{1} \log ^{q} n+c_{2}\right\} \\
& \quad+\mathbb{P}[\mathbf{U} \notin \mathcal{T}(n, \alpha, p)] \\
& \leq \mathbb{P}\left[\mathbf{U} \in \mathcal{T}(n, \alpha, p), \Lambda_{\mathbf{Y}^{\star}}\left(\mathbf{X}, \hat{\lambda}^{\star}(\mathbf{X}), d\right)-\jmath \mathbf{X}(\mathbf{X}, d)>C \log n\right] \\
& \quad+\mathbb{P}[\mathbf{U} \notin \mathcal{T}(n, \alpha, p)] \\
& \leq \frac{K}{(\log n)^{c \alpha}},
\end{aligned}
$$

where 273) is by Lemma 8 , (274) is by 266; (275) is by the choice of $\gamma$ in (265), $C_{d}$ in (270) and $q>1$; (276) holds due to Lemma 9 and (125) in Theorem 11, $K=A_{1}+C_{2}$, where $A_{1}>0$ is the constant in Theorem 11 .

\section{B. Proof of Theorem 11}

Proof. We first show that the probability mass of the set $\mathcal{T}(n, \alpha, p)$ is large. Choosing $\eta=\sqrt{\frac{\alpha \log \log n}{n}}$ in Theorem 10 
implies that the condition (121) is violated with probability at most

$$
2 \max \left\{\frac{e^{2}}{(\log n)^{c \alpha}}, \frac{2}{e^{\frac{n}{64}}}\right\}=\frac{2 e^{2}}{(\log n)^{c \alpha}},
$$

for $n \geq N_{1}$ for some $N_{1} \in \mathbb{N}$. We apply the Berry-Esséen theorem to bound the probability of violating condition (123). For $i \in[n]$, note that $G_{i} \triangleq \frac{X_{i}}{\sigma_{i}} \sim \mathcal{N}(0,1)$, since $X_{i} \sim$ $\mathcal{N}\left(0, \sigma_{i}^{2}\right)$ by (44). For each $k=1,2,3$, applying the BerryEsséen theorem 8 to the zero-mean and bounded finite moments random variables $G_{i}^{2 k}-(2 k-1) ! !, i \in[n]$, we obtain

$$
\mathbb{P}\left[\left|\frac{1}{n} \sum_{i=1}^{n} G_{i}^{2 k}-(2 k-1) ! !\right|>2\right] \leq 2 Q\left(\frac{2 \sqrt{n}}{r_{k}}\right)+\frac{12 T_{k}}{r_{k}^{3} \sqrt{n}},
$$

where $r_{k}^{2}$ is the variance of $G_{i}^{2 k}-(2 k-1) ! !$, and $T_{k}$ is the third absolute moment of $G_{i}^{2 k}-(2 k-1) ! !$, and $r_{k}$ and $T_{k}$ are both constants. Therefore, there exists $N_{2} \in \mathbb{N}$ large enough and a constant $A_{1}^{\prime}$ such that for $n \geq N_{2}$,

$$
\mathbb{P}\left[\left|\frac{1}{n} \sum_{i=1}^{n} G_{i}^{2 k}-(2 k-1) ! !\right|>2\right] \leq \frac{A_{1}^{\prime}}{\sqrt{n}},
$$

which means that condition $(123)$ is violated with probability at most $\frac{3 A_{1}^{\prime}}{\sqrt{n}}$ for $n \geq N_{2}$.

Verifying that the condition 122 is satisfied with high probability is more involved. The high-level procedure is the following. The expressions for $m_{i}(\mathbf{x}), i \in[n]$ in 120$)$ are derived using the characterization of the optimizer of the optimization problem (49) in Lemma 5 in Appendix B-C1 Then we approximate $m_{i}(\mathbf{x})$ using $\bar{m}_{i}\left(x_{i}\right)$, for which it is easier to show a concentration bound of the form (122). At the end, the differences between $m_{i}(\mathbf{x})$ and $\bar{m}_{i}\left(x_{i}\right)$ are shown to be sufficiently small, and (122) follows. We start with a closer look at the optimizer $\hat{\mathbf{F}}^{\star}$ in $\mathbb{R}\left(\hat{\mathbf{X}}, \mathbf{Y}^{\star}, d\right)$. Recall the distributions of $\mathbf{X}$ and $\mathbf{Y}^{\star}$ in (214)-217), and the distribution of $\hat{\mathbf{X}}$ in (119). An application of Lemma 5 in Appendix B-C1 to $\mathbb{R}\left(\hat{\mathbf{X}}, \mathbf{Y}^{\star}, d\right)$ yields that for any $\mathbf{x} \in \mathbb{R}^{n}$,

$$
\begin{aligned}
P_{\hat{\mathbf{F}}^{\star} \mid \hat{\mathbf{X}}=\mathbf{x}} & =\prod_{i=1}^{n} P_{\hat{F}_{i}^{\star} \mid \hat{X}_{i}=x_{i}}, \\
\hat{F}_{i}^{\star} \mid \hat{X}_{i}=x_{i} & \sim \mathcal{N}\left(\frac{2 \hat{\lambda}^{\star}(\mathbf{x}) \nu_{i}^{2} x_{i}}{1+2 \hat{\lambda}^{\star}(\mathbf{x}) \nu_{i}^{2}}, \frac{\nu_{i}^{2}}{1+2 \hat{\lambda}^{\star}(\mathbf{x}) \nu_{i}^{2}}\right),
\end{aligned}
$$

where $\hat{\lambda}^{\star}(\mathbf{x})$ is given by (128). Then from (281) and the definition of $m_{i}(\mathbf{x})$ in 1120 , it is straightforward to obtain the following expression for $m_{i}(\mathbf{x}), i \in[n]$ :

$$
m_{i}(\mathbf{x})=\frac{\nu_{i}^{2}}{1+2 \hat{\lambda}^{\star}(\mathbf{x}) \nu_{i}^{2}}+\frac{x_{i}^{2}}{\left(1+2 \hat{\lambda}^{\star}(\mathbf{x}) \nu_{i}^{2}\right)^{2}},
$$

which is hard to analyze since $\hat{\lambda}^{\star}(\mathbf{x})$ does not have a simple formula. We instead consider $\bar{m}_{i}\left(x_{i}\right), i \in[n]$, defined as

$$
\bar{m}_{i}\left(x_{i}\right) \triangleq \frac{\nu_{i}^{2}}{1+2 \lambda^{\star \star} \nu_{i}^{2}}+\frac{x_{i}^{2}}{\left(1+2 \lambda^{\star \star} \nu_{i}^{2}\right)^{2}},
$$

\footnotetext{
${ }^{8}$ The Berry-Esséen theorem suffices here, though tighter bounds are possible via other concentration inequalities, say Chernoff's bound.
}

which is obtained by replacing $\hat{\lambda}^{\star}(\mathbf{x})$ with $\lambda^{\star \star}$ in 282). The random variable $\bar{m}_{i}\left(X_{i}\right)$ is much easier to analyze, since $\lambda^{\star \star}=$ $\frac{1}{2 \theta_{n}}$ by Lemma 6 in Appendix B-C2 and 283 is simplified as

$$
\bar{m}_{i}\left(x_{i}\right)=\frac{\left(\min \left(\sigma_{i}^{2}, \theta_{n}\right)\right)^{2}}{\sigma_{i}^{2}}\left(\frac{x_{i}^{2}}{\sigma_{i}^{2}}-1\right)+\min \left(\sigma_{i}^{2}, \theta_{n}\right) .
$$

We will control the difference between $m_{i}(\mathbf{x})$ and $\bar{m}_{i}\left(x_{i}\right)$ by bounding $\left|\lambda^{\star \star}-\hat{\lambda}^{\star}(\mathbf{x})\right|$. Indeed, a lengthy elementary calculation, deferred to the end of the proof, shows that there exists $N_{3} \in \mathbb{N}$ and a constant $A_{1}^{\prime \prime}>0$ (depending only on d) such that for any $n \geq N_{3}$, any $\mathbf{x} \in \mathbb{R}^{n}$ satisfying 121 and 123,

$$
\left|\frac{1}{n} \sum_{i=1}^{n} \bar{m}_{i}\left(x_{i}\right)-\frac{1}{n} \sum_{i=1}^{n} m_{i}(\mathbf{x})\right| \leq A_{1}^{\prime \prime} \sqrt{\frac{\alpha \log \log n}{n}} .
$$

We proceed to explain how to apply the Berry-Esséen theorem to obtain the following bound: there exists a constant $A_{1}^{\prime \prime \prime}>0$ and $N_{4} \in \mathbb{N}$ such that for any $\omega>0$ and $n \geq N_{4}$,

$$
\mathbb{P}\left[\left|\frac{1}{n} \sum_{i=1}^{n} \bar{m}_{i}\left(X_{i}\right)-d\right| \geq \omega \sqrt{\frac{\log \log n}{n}}\right] \leq \frac{A_{1}^{\prime \prime \prime}}{(\log n)^{\frac{\omega^{2}}{2 \beta^{2}}}},
$$

where

$$
\beta^{2}=\frac{1}{n} \sum_{i=1}^{n} \operatorname{Var}\left[\bar{m}_{i}\left(X_{i}\right)\right] .
$$

To this end, first note from (284) and (15) that

$$
\frac{1}{n} \sum_{i=1}^{n} \mathbb{E}\left[\bar{m}_{i}\left(X_{i}\right)\right]=\frac{1}{n} \sum_{i=1}^{n} \min \left(\sigma_{i}^{2}, \theta_{n}\right)=d,
$$

An application of the Berry-Esséen theorem to $\bar{m}_{i}\left(X_{i}\right)-$ $\min \left(\sigma_{i}^{2}, \theta_{n}\right)$ yields

$$
\begin{aligned}
& \mathbb{P}\left[\left|\frac{1}{n} \sum_{i=1}^{n} \bar{m}_{i}\left(X_{i}\right)-d\right| \geq \omega \sqrt{\frac{\log \log n}{n}}\right] \\
\leq & 2 Q\left(\frac{\omega \sqrt{\log \log n}}{\beta}\right)+\frac{12 T}{\beta^{3} \sqrt{n}} \\
\leq & \frac{2}{(\log n)^{\frac{\omega^{2}}{2 \beta^{2}}}}+\frac{12 T}{\beta^{3} \sqrt{n}},
\end{aligned}
$$

where $T=\frac{1}{n} \sum_{i=1}^{n} \mathbb{E}\left[\left|\bar{m}_{i}\left(X_{i}\right)-\min \left(\sigma_{i}^{2}, \theta_{n}\right)\right|^{3}\right]$ is bounded by constants, and it is easy to check that $0<\beta_{d}<\beta \leq$ $\frac{\sqrt{2} \sigma^{2}}{(1-a)^{2}}$ for some constant $\beta_{d}>0$, which depends on $d$ only. Therefore, 286 follows.

Now, we combine 285 and 286 to conclude that the condition 122 is satisfied with high probability. Define the set $\mathcal{L} \subset \mathbb{R}^{n}$ as

$$
\mathcal{L} \triangleq\left\{\mathbf{u} \in \mathbb{R}^{n}: \mathbf{u} \text { satisfies 121) and 123) }\right\},
$$

then 277) and 279) imply that

$$
\mathbb{P}\left[\mathbf{U} \in \mathcal{L}^{c}\right] \leq \frac{2 e^{2}}{(\log n)^{c \alpha}}+\frac{3 A_{1}^{\prime}}{\sqrt{n}} .
$$


Finally, we have

$$
\begin{aligned}
& \mathbb{P}\left[\left|\frac{1}{n} \sum_{i=1}^{n} m_{i}(\mathbf{X})-d\right| \geq p \sqrt{\frac{\alpha \log \log n}{n}}\right] \\
\leq & \mathbb{P}\left\{\left|\frac{1}{n} \sum_{i=1}^{n} \bar{m}_{i}\left(X_{i}\right)-\frac{1}{n} \sum_{i=1}^{n} m_{i}(\mathbf{X})\right|+\right. \\
= & \mathbb{P}\left[\cdot, \mathbf{U} \in \mathcal{L} \sum_{i=1}^{n} \bar{m}_{i}\left(X_{i}\right)-d \mid \geq p \sqrt{\frac{\alpha \log \log n}{n}}\right\} \\
\leq & \mathbb{P}\left[\left|\frac{1}{n} \sum_{i=1}^{n} \bar{m}_{i}\left(X_{i}\right)-d\right| \geq\left(p-A_{1}^{\prime \prime}\right) \sqrt{\frac{\alpha \log \log n}{n}}\right] \\
\leq & \frac{\left.+\mathcal{L}^{c}\right]}{(\log n)^{\frac{\left(p-A_{1}^{\prime \prime}\right)^{2} \alpha}{2 \beta^{2}}}}+\frac{2 e^{2}}{(\log n)^{c \alpha}}+\frac{3 A_{1}^{\prime}}{\sqrt{n}},
\end{aligned}
$$

where 294 is due to triangle inequality; 296 holds by 285; 297) follows from 286 for $p>A_{1}^{\prime \prime}$. Hence, for any $p$ in 297] such that

$$
p \geq A_{1}^{\prime \prime}+\frac{2 \sqrt{c} \sigma^{2}}{(1-a)^{2}},
$$

we conclude from 2977 that there exists a constant $\tilde{A}_{1}>0$ and $N_{5} \in \mathbb{N}$ large enough such that for $n \geq N_{5}$,

$$
\mathbb{P}\left[\left|\frac{1}{n} \sum_{i=1}^{n} m_{i}(\mathbf{X})-d\right| \geq p \sqrt{\frac{\alpha \log \log n}{n}}\right] \leq \frac{\tilde{A}_{1}}{(\log n)^{c \alpha}} .
$$

Therefore, by 277], 279) and 299, there exists a constant $A_{1}>0$ and $N(d, \alpha) \triangleq \max _{j \in[5]} N_{j}$ such that for $n \geq N(d, \alpha)$,

$$
\mathbb{P}[\mathbf{U} \in \mathcal{T}(n, \alpha, p)] \geq 1-\frac{A_{1}}{(\log n)^{c \alpha}},
$$

where $p$ satisfies 298.

Next, we show (126), which states that the proxy variances are good approximations to $\sigma_{i}^{2}, i \in[n]$. To this end, for any $\mathbf{u} \in \mathcal{T}(n, \alpha, p)$, note that for any $i \in[n]$,

$$
\left|\hat{\sigma}_{i}^{2}-\sigma_{i}^{2}\right| \leq\left|\hat{\sigma}_{i}^{2}-\frac{\sigma^{2}}{\xi_{i}}\right|+\left|\frac{\sigma^{2}}{\xi_{i}}-\sigma_{i}^{2}\right|,
$$

where $\xi_{i}$ is given in (176). From (177), (185), and (45), we have

$$
\left|\frac{\sigma^{2}}{\xi_{i}}-\sigma_{i}^{2}\right| \leq \frac{2 a \pi \sigma^{2}}{(1-a)^{4} n} .
$$

On the other hand, let $\phi_{i} \triangleq \frac{i \pi}{n+1}$ and denote by $\varphi(t)$ the function

$$
\varphi(t) \triangleq \frac{\sigma^{2}}{1+t^{2}-2 t \cos \phi_{i}} .
$$

By Taylor expansion and triangle inequality, we have

$$
\begin{aligned}
\left|\hat{\sigma}_{i}^{2}-\frac{\sigma^{2}}{\xi_{i}}\right| & =|\varphi(\hat{a}(\mathbf{u}))-\varphi(a)| \\
& \leq\left|\varphi^{\prime}(a)\right||\hat{a}(\mathbf{u})-a|+o(|\hat{a}(\mathbf{u})-a|) .
\end{aligned}
$$

Hence, combining (301), (302) and (305), we conclude that there exists a constant $A_{2}>0$ and $N \in \mathbb{N}$ such that (126) holds for any $\mathbf{u} \in \mathcal{T}(n, \alpha, p)$ and any $n \geq N$.

Finally, the bound (127) follows immediately from a direct application of Theorem 16 to 126 .

Elementary calculations to show 285): From 282 and (283), we have

$$
\begin{aligned}
& \frac{1}{n} \sum_{i=1}^{n} \bar{m}_{i}\left(x_{i}\right)-\frac{1}{n} \sum_{i=1}^{n} m_{i}(\mathbf{x}) \\
= & \frac{1}{n} \sum_{i=1}^{n} \frac{2 \nu_{i}^{4}\left(\hat{\lambda}^{\star}(\mathbf{x})-\lambda^{\star \star}\right)}{\left(1+2 \hat{\lambda}^{\star}(\mathbf{x}) \nu_{i}^{2}\right)\left(1+2 \lambda^{\star \star} \nu_{i}^{2}\right)}+ \\
& \frac{1}{n} \sum_{i=1}^{n} \frac{2 x_{i}^{2} \nu_{i}^{2}\left(2+2 \hat{\lambda}^{\star}(\mathbf{x}) \nu_{i}^{2}+2 \lambda^{\star \star} \nu_{i}^{2}\right)\left(\hat{\lambda}^{\star}(\mathbf{x})-\lambda^{\star \star}\right)}{\left(1+2 \hat{\lambda}^{\star}(\mathbf{x}) \nu_{i}^{2}\right)^{2}\left(1+2 \lambda^{\star \star} \nu_{i}^{2}\right)^{2}} .
\end{aligned}
$$

Note that (127) implies that there exists $N_{1}^{\prime} \in \mathbb{N}$ such that for any $\mathbf{u} \in \mathcal{T}(n, \alpha, p)$ and $\mathbf{x}=\mathrm{S}^{T} \mathbf{u}$, any $n \geq N_{1}^{\prime}$, and any $i \in[n]$,

$$
\left|\left(1+2 \hat{\lambda}^{\star}(\mathbf{x}) \nu_{i}^{2}\right)-\left(1+2 \lambda^{\star \star} \nu_{i}^{2}\right)\right| \leq \frac{1+2 \lambda^{\star \star} \nu_{i}^{2}}{2},
$$

which can be equivalently rewritten as

$$
\frac{\sigma_{i}^{2}}{2 \theta_{n}} \leq 1+2 \hat{\lambda}^{\star}(\mathbf{x}) \nu_{i}^{2} \leq \frac{3 \sigma_{i}^{2}}{2 \theta_{n}}
$$

since $1+2 \lambda^{\star \star} \nu_{i}^{2}=\frac{\sigma_{i}^{2}}{\theta_{n}}, \forall i \in[n]$ by $\lambda^{\star \star}=1 /\left(2 \theta_{n}\right)$ in Lemma 6 in Appendix B-C2 Continuing the inequality in (307) yields

$$
\begin{aligned}
& \left|\frac{1}{n} \sum_{i=1}^{n} \bar{m}_{i}\left(x_{i}\right)-\frac{1}{n} \sum_{i=1}^{n} m_{i}(\mathbf{x})\right| \\
\leq & \frac{1}{n} \sum_{i=1}^{n} \frac{4 \nu_{i}^{4} \theta_{n}^{2}}{\sigma_{i}^{4}}\left|\hat{\lambda}^{\star}(\mathbf{x})-\lambda^{\star \star}\right|+\frac{1}{n} \sum_{i=1}^{n} \frac{20 x_{i}^{2} \nu_{i}^{2} \theta_{n}^{3}}{\sigma_{i}^{6}}\left|\hat{\lambda}^{\star}(\mathbf{x})-\lambda^{\star \star}\right|
\end{aligned}
$$

$\leq\left[\frac{1}{n} \sum_{i=1}^{n} \frac{\sigma_{i}^{4}}{4}+\frac{1}{n} \sum_{i=1}^{n} \frac{5 \theta_{n}^{2} x_{i}^{2}}{\sigma_{i}^{2}}\right]\left|\hat{\lambda}^{\star}(\mathbf{x})-\lambda^{\star \star}\right|$

$\leq A_{1}^{\prime \prime} \sqrt{\frac{\alpha \log \log n}{n}}$,

where 311 is by plugging the bound 309 into 307 ; 312 is by

$$
\nu_{i}^{2} \theta_{n}=\left(\sigma_{i}^{2}-\theta_{n}\right) \theta_{n} \leq \frac{\sigma_{i}^{4}}{4} ;
$$

(313) holds for some constant $A_{1}^{\prime \prime}>0$ by the moments bound in (123), the bound (127), and

$$
\sigma_{i}^{4}=\frac{\sigma^{4}}{\mu_{i}^{2}} \leq \frac{\sigma^{4}}{(1-a)^{4}},
$$

for any $i \in[n]$, which follows from 189$]$. 


\section{Proof of Lemma 9}

Proof. Since $Y_{1}^{\star}, \ldots, Y_{n}^{\star}$ are independent and the distortion measure $\mathrm{d}(\cdot, \cdot)$ is separable, we know that the generalized tilted information splits as

$$
\begin{aligned}
\Lambda_{\mathbf{Y}^{\star}}\left(\mathbf{x}, \hat{\lambda}^{\star}(\mathbf{x}), d\right) & =\sum_{i=1}^{n} \Lambda_{Y_{i}^{\star}}\left(x_{i}, \hat{\lambda}^{\star}(\mathbf{x}), d_{i}\right), \\
\Lambda_{\mathbf{Y}^{\star}}\left(\mathbf{x}, \lambda^{\star \star}, d\right) & =\sum_{i=1}^{n} \Lambda_{Y_{i}^{\star}}\left(x_{i}, \lambda^{\star \star}, d_{i}\right),
\end{aligned}
$$

where $d_{i}=\min \left(\sigma_{i}^{2}, \theta_{n}\right)$, and $\theta_{n}>0$ is the water level matched to $d$ via the $n$-th order reverse waterfilling (15) over $\sigma_{i}^{2}, i \in[n]$. The rest of the proof of Lemma 9 is almost the same as [13 Eq. (317)-(340)] except that we need to replace the set $F_{n}$ in [13] by our typical set $\mathcal{T}(n, \alpha, p)$.

\section{Proof of Lemma 10}

Proof. Due to Lemma 5 in B-C1, for any $\mathrm{x} \in \mathbb{R}^{n}$, we can write the random variable

$$
d\left(\mathbf{x}, \hat{\mathbf{F}}^{\star}\right) \mid \hat{\mathbf{X}}=\mathbf{x}
$$

as a sum of independent random variables, to which the BerryEsséen theorem applies. Specifically, the distribution of the optimizer of $\mathbb{R}\left(\hat{\mathbf{X}}, \mathbf{Y}^{\star}, d\right)$ is given in (280) and 281). Thus, we have

$$
\left[\mathrm{d}\left(\mathbf{x}, \hat{\mathbf{F}}^{\star}\right) \mid \hat{\mathbf{X}}=\mathbf{x}\right]=\frac{1}{n} \sum_{i=1}^{n} M_{i}(\mathbf{x}),
$$

where the random variables $M_{i}(\mathbf{x})$, for $i \in[n]$, are defined as

$$
M_{i}(\mathbf{x}) \triangleq\left(\hat{F}_{i}^{\star}-x_{i}\right)^{2} \mid \hat{X}_{i}=x_{i},
$$

and by 281

$$
M_{i}(\mathbf{x}) \sim \mathcal{N}\left(\frac{-x_{i}}{1+2 \hat{\lambda}^{\star}(\mathbf{x}) \nu_{i}^{2}}, \frac{\nu_{i}^{2}}{1+2 \hat{\lambda}^{\star}(\mathbf{x}) \nu_{i}^{2}}\right) .
$$

Hence $d\left(\mathbf{x}, \hat{\mathbf{F}}^{\star}\right) \mid\{\hat{\mathbf{X}}=\mathbf{x}\}$ follows the noncentral $\chi^{2}$ distribution with $n$-degrees of freedom.

For any $d \in\left(0, d_{\max }\right), \epsilon \in(0,1)$ and $\alpha>0$, let $\mathcal{T}(n, \alpha, p)$ be the set in Theorem 11. For any $\mathbf{u} \in \mathcal{T}(n, \alpha, p)$, and $\mathbf{x}=$ $\mathrm{S}^{T} \mathbf{u}$, let $\hat{\mathbf{X}}$ be defined in (119). The Berry-Esséen theorem yields

$$
\begin{aligned}
& \mathbb{P}\left[d-\gamma<\mathrm{d}\left(\mathbf{x}, \hat{\mathbf{F}}^{\star}\right) \leq d \mid \hat{\mathbf{X}}=\mathbf{x}\right] \\
= & \mathbb{P}\left[\frac{n d-n \gamma-\sum_{i=1}^{n} m_{i}(\mathbf{x})}{s \sqrt{n}}<\frac{1}{s \sqrt{n}} \sum_{i=1}^{n}\left(M_{i}^{2}-m_{i}(\mathbf{x})\right)\right. \\
\leq & \left.\frac{n d-\sum_{i=1}^{n} m_{i}(\mathbf{x})}{s \sqrt{n}}\right] \\
\geq & \Phi\left(\frac{n d-\sum_{i=1}^{n} m_{i}(\mathbf{x})}{s \sqrt{n}}\right)- \\
& \Phi\left(\frac{n d-n \gamma-\sum_{i=1}^{n} m_{i}(\mathbf{x})}{s \sqrt{n}}\right)-\frac{12 t}{s^{3} \sqrt{n}}
\end{aligned}
$$

where $\Phi$ is the cdf of the standard normal distribution, and $m_{i}(\mathbf{x})$ are expectations of $M_{i}(\mathbf{x}), i \in[n]$, defined in (120), and

$$
\begin{aligned}
s^{2} & \triangleq \frac{1}{n} \sum_{i=1}^{n} \operatorname{Var}\left[M_{i}^{2}(\mathbf{x})\right], \\
t & \triangleq \frac{1}{n} \sum_{i=1}^{n} \mathbb{E}\left[\left|M_{i}^{2}(\mathbf{x})-m_{i}(\mathbf{x})\right|^{3}\right] .
\end{aligned}
$$

By the mean value theorem, 324) equals

$$
\frac{n \gamma}{s \sqrt{n} \sqrt{2 \pi}} e^{-\frac{b^{2}}{2}}-\frac{12 t}{s^{3} \sqrt{n}},
$$

for some $b$ satisfying

$$
\frac{n d-\sum_{i=1}^{n} m_{i}(\mathbf{x})-n \gamma}{s \sqrt{n}} \leq b \leq \frac{n d-\sum_{i=1}^{n} m_{i}(\mathbf{x})}{s \sqrt{n}} .
$$

To further lower-bound (327), we bound $b^{2}$ as follows.

$$
\begin{aligned}
b^{2} & \leq\left(\frac{n d-\sum_{i=1}^{n} m_{i}(\mathbf{x})}{s \sqrt{n}}\right)^{2}+\left(\frac{n \gamma}{s \sqrt{n}}\right)^{2} \\
& \leq \frac{p^{2} \alpha \log \log n}{s^{2}}+\frac{\log ^{2 q} n}{s^{2} n} \\
& \leq \frac{2 p^{2} \alpha \log \log n}{c_{s}^{2}}
\end{aligned}
$$

where (330) is by (122) and by choosing $\gamma$ as in (265), and the constant $q$ in 265) is chosen to be

$$
q \triangleq \frac{p^{2} \alpha}{\mathrm{c}_{s}^{2}}+1
$$

and the constant $\mathrm{c}_{s}>0$ is a lower bound of $s$, whose existence is justified below at the end of the proof; 331 holds for $n \geq N_{1}$ for some $N_{1} \in \mathbb{N}$. Using [331, we can further lowerbound (327)

$$
\begin{aligned}
& \left(\frac{\log ^{q} n}{s \sqrt{2 \pi}}(\log n)^{-\frac{p^{2} \alpha}{c_{s}^{2}}}-\frac{12 t}{s^{3}}\right) \frac{1}{\sqrt{n}} \\
= & \left(\frac{\log n}{s \sqrt{2 \pi}}-\frac{12 t}{s^{3}}\right) \frac{1}{\sqrt{n}} \\
\geq & \frac{C_{1}}{\sqrt{n}},
\end{aligned}
$$

where (335) holds for $n \geq N_{2}$ for some large enough $N_{2} \in \mathbb{N}$ and some constant $C_{1}>0$.

Finally, to justify that $a$ and $t$, defined in (325) and (326), are bounded as we assumed in obtaining (331) and (335), we observe that $M_{i}$ has bounded positive finite-order moments. Indeed, to see that $s$ is bounded, from (321), for $i \in[n]$, we compute

$$
\operatorname{Var}\left[M_{i}^{2}(\mathbf{x})\right]=\frac{4 x_{i}^{2} \nu_{i}^{2}}{\left(1+2 \hat{\lambda}^{\star}(\mathbf{x}) \nu_{i}^{2}\right)^{3}}+\frac{2 \nu_{i}^{4}}{\left(1+2 \hat{\lambda}^{\star}(\mathbf{x}) \nu_{i}^{2}\right)^{2}} .
$$

Then, using (309) to bound $1+2 \hat{\lambda}^{\star}(\mathbf{x}) \nu_{i}^{2}$ and 123 to bound $x_{i}^{2}$, we can lower- and upper-bound $s$ by positive constants; $t$ is bounded similarly. 


\section{APPENDIX E}

Proofs in SECTION IV-B

\section{A. Proof of Theorem 10}

The proof is based on the following two lemmas.

Lemma 11. Define the set

$$
\mathcal{E}_{\rho} \triangleq\left\{\mathbf{u} \in \mathbb{R}^{n}: \sum_{i=2}^{n} u_{i-1}^{2}>(n-1) \rho\right\}
$$

where

$$
\rho \triangleq \frac{\sigma^{2}}{2(1+a)^{2}} .
$$

Then, for $n \geq 2$, it holds that

$$
\mathbb{P}\left[\mathbf{U} \notin \mathcal{E}_{\rho}\right] \leq 2 e^{-\frac{n}{64}},
$$

where $\mathbf{U}$ follows the Gauss-Markov source law (2),

Proof. Appendix E-B

Lemma 12. Consider $\rho$ given by (338), and any $\eta$ satisfying (116). For any $n \geq \frac{3-a}{1-a}$,

$$
\mathbb{P}\left[\left|\sum_{i=2}^{n} U_{i-1} Z_{i}\right|>\eta(n-1) \rho\right] \leq e^{2} e^{-c n \eta^{2}},
$$

where $c$ is given by (100).

Proof. Appendix E-C

Proof of Theorem 10. For any $\eta$ satisfying (116) and any $n \geq$ $\frac{3-a}{1-a}$,

$$
\begin{aligned}
& \mathbb{P}[|\hat{a}(\mathbf{U})-a|>\eta] \\
= & \mathbb{P}\left[\left|\sum_{i=2}^{n} U_{i-1}\left(U_{i}-a U_{i-1}\right)\right|>\eta \sum_{i=2}^{n} U_{i-1}^{2}\right] \\
= & \mathbb{P}\left[\left|\sum_{i=2}^{n} U_{i-1} Z_{i}\right|>\eta \sum_{i=2}^{n} U_{i-1}^{2}\right] \\
= & \mathbb{P}\left[\left|\sum_{i=2}^{n} U_{i-1} Z_{i}\right|>\eta \sum_{i=2}^{n} U_{i-1}^{2} \mid \mathbf{U} \in \mathcal{E}_{\rho}\right] \mathbb{P}\left[\mathbf{U} \in \mathcal{E}_{\rho}\right]+ \\
& \mathbb{P}\left[\left|\sum_{i=2}^{n} U_{i-1} Z_{i}\right|>\eta \sum_{i=2}^{n} U_{i-1}^{2} \mid \mathbf{U} \notin \mathcal{E}_{\rho}\right] \mathbb{P}\left[\mathbf{U} \notin \mathcal{E}_{\rho}\right] \\
\leq & \mathbb{P}\left[\left|\sum_{i=2}^{n} U_{i-1} Z_{i}\right|>\eta(n-1) \rho\right]+\mathbb{P}\left[\mathbf{U} \notin \mathcal{E}_{\rho}\right] \\
\leq & 2 \max \left\{e^{2} e^{-c n \eta^{2}}, 2 e^{-\frac{n}{64}}\right\}
\end{aligned}
$$

where $(342)$ holds by plugging $(110)$ into $(341)$ and rearranging terms; $(343)$ is by the definition of the Gauss-Markov source in (2); (344) is by the law of total expectation; 345) is by the definition of $\mathcal{E}_{\rho}$ in 337); 346 is by Lemma 11 and Lemma 12

\section{B. Proof of Lemma 11}

The proof is based on the Chernoff bound. To simplify notations, denote by $\tilde{\mathbf{U}} \triangleq\left(U_{1}, \ldots, U_{n-1}\right)$ and $\tilde{\mathbf{Z}} \triangleq\left(Z_{1}, \ldots, Z_{n-1}\right)$ the $(n-1)$ random vectors generated according to the GaussMarkov source model (2). Denote by $\tilde{\mathrm{A}}$ the $(n-1) \times(n-1)$ matrix in (39) such that $\tilde{\mathbf{Z}}=\tilde{\mathrm{A}} \tilde{\mathbf{U}}$. Let $\tilde{\mu}_{1} \leq \ldots \leq \tilde{\mu}_{n-1}$ be the eigenvalues of $\tilde{A} \tilde{A}^{T} 9$ Let $\tilde{S}$ be the unitary matrix in the eigendecomposition $\left(\tilde{A} \tilde{A}^{T}\right)^{-1}=\tilde{S} \tilde{\Lambda} \tilde{S} \tilde{S}^{T}$, where

$$
\tilde{\Lambda}=\operatorname{diag}\left(1 / \tilde{\mu}_{1}, \ldots, 1 / \tilde{\mu}_{n-1}\right) .
$$

Define

$$
\mathbf{G} \triangleq \tilde{\mathrm{S}}^{T} \tilde{\mathbf{Z}}=\tilde{\mathrm{S}}^{T} \tilde{\mathrm{A}}^{-1} \tilde{\mathbf{U}}
$$

The covariance matrix of $\mathbf{G}$ is given by

$$
\Sigma_{\mathbf{G}}=\sigma^{2} \mathrm{l} \text {. }
$$

That is, $G_{i} \sim \mathcal{N}\left(0, \sigma^{2}\right)$ are independent zero-mean Gaussian random variables for $i \in[n-1]$. Therefore,

$$
\begin{aligned}
\mathbb{P}\left[\mathbf{U} \notin \mathcal{E}_{\rho}\right] & =\mathbb{P}\left[\tilde{\mathbf{U}}^{T} \tilde{\mathbf{U}} \leq(n-1) \rho\right] \\
& =\mathbb{P}\left[\sum_{i=1}^{n-1} \frac{g_{i}^{2}}{\tilde{\mu}_{i}} \leq(n-1) \rho\right] \\
& \leq \mathbb{P}\left[\sum_{i=1}^{n-1} g_{i}^{2} \leq(n-1) \rho(1+a)^{2}\right] \\
& \leq 2 e^{-\frac{n-1}{32}} \\
& \leq 2 e^{-\frac{n}{64}},
\end{aligned}
$$

where (351) follows by plugging $(348)$ into $(350)$; 352 ) holds since $\tilde{\mu}_{i} \leq(1+a)^{2}$ due to (189); (353) $)$ is by applying the Chernoff bound in Theorem 13 with $t=1 / 2$ and $n-1$; 354 holds for any $n \geq 2$.

\section{Proof of Lemma 12}

The key tool is the following concentration result on multilinear polynomials in random variables with bounded moments [24].

Theorem 17 ( [24, Th. 1.4], particularized to Gaussian random variables). Let $\mathbf{Z}=\left(Z_{1}, \ldots, Z_{n}\right)$, where $Z_{1}, \ldots, Z_{n}$ are i.i.d. random variables and $Z_{1} \sim \mathcal{N}\left(0, \sigma^{2}\right)$, and let $f: \mathbb{R}^{n} \rightarrow \mathbb{R}$ be a multilinear polynomial of total degree $m$. For any $t>0$, it holds that

$$
\begin{aligned}
& \mathbb{P}[|f(\mathbf{Z})-\mathbb{E}[f(\mathbf{Z})]| \geq t] \\
\leq & e^{2} \cdot \max \left\{\max _{r \in[m]} e^{-\frac{t^{2}}{\theta_{0} \theta_{r} L^{r} J^{m}}}, \max _{r \in[m]} e^{-\left(\frac{t}{\theta_{r} L^{r} J^{m}}\right)^{1 / r}}\right\},
\end{aligned}
$$

where $L$ and $J$ are absolute constants, and $L=1.44 \mathbb{E}\left[\left|Z_{1}\right|\right] \approx$ $1.15 \sigma$ for $Z_{1} \sim \mathcal{N}\left(0, \sigma^{2}\right)$, and $\theta_{i}, i=0, \ldots, m$, determined by the function $f$ and the random variable $Z_{1}$, are specified in Definition 3 in Appendix E-D below.

In our application below, we will be dealing with a multilinear polynomial of total degree $m=2$. We list the bounds 


\begin{tabular}{|c|c|}
\hline Bounds in order & Exact bounds \\
\hline$\theta_{0}=\Theta(n)$ & $\frac{2 \sigma^{2}}{(1-a) \pi}\left(n-\frac{1}{1-a}\right) \leq \theta_{0} \leq \frac{2 \sigma^{2} n}{(1-a) \pi}$ \\
\hline$\theta_{1}=\Theta(1)$ & $0 \leq \theta_{1} \leq \frac{2 \sqrt{2} \sigma}{\sqrt{\pi}(1-a)}$ \\
\hline$\theta_{2}=\Theta(1)$ & $\theta_{2}=0$ \\
\hline
\end{tabular}

TABLE I: Bounds on $\theta_{0}, \theta_{1}, \theta_{2}$.

on $\theta_{0}, \theta_{1}, \theta_{2}$ in Table I] while defer the detailed derivations to Appendix E-D.

Proof of Lemma 12. We first rewrite the summation in (340) as a multilinear function. Recall from the Gauss-Markov source model 2 that for any $i \in[n]$,

$$
U_{i}=\sum_{j=1}^{i} a^{i-j} Z_{j}
$$

which implies

$$
\begin{aligned}
\sum_{i=2}^{n} U_{i-1} Z_{i} & =\sum_{i=2}^{n} \sum_{j=1}^{i-1} a^{i-1-j} Z_{j} Z_{i} \\
& =\sum_{j=1}^{n-1} \sum_{i=j+1}^{n} a^{i-1-j} Z_{j} Z_{i} .
\end{aligned}
$$

Define

$$
f\left(z_{1}, \ldots, z_{n}\right) \triangleq \sum_{j=1}^{n-1} \sum_{i=j+1}^{n} a^{i-1-j} z_{j} z_{i},
$$

which is a multilinear function in $z_{1}, \ldots, z_{n}$ with total degree $m=2$. Note that $\mathbb{E}\left[f\left(Z_{1}, \ldots, Z_{n}\right)\right]=0$ by the independence of $Z_{i}$ 's. By Theorem 17 and bounds in Table I, we have

$$
\begin{aligned}
& \mathbb{P}\left[\left|\sum_{i=2}^{n} U_{i-1} Z_{i}\right|>\eta(n-1) \rho\right] \\
= & \mathbb{P}\left[\left|f\left(Z_{1}, \ldots, Z_{n}\right)\right|>\eta(n-1) \rho\right] \\
\leq & e^{2} \max \left\{e^{-\frac{\eta^{2}(n-1)^{2} \rho^{2}}{\theta_{0} \theta_{1} L R^{2}}}, e^{-\frac{\eta(n-1) \rho}{\theta_{1} L R^{2}}}\right\} \\
\leq & e^{2} e^{-c n \eta^{2}},
\end{aligned}
$$

where 361) is by Theorem 17 and $\theta_{2}=0$; 362 holds by comparing the two exponents inside the maximum using the bounds in Table I (116), 338) and $n \geq \frac{3-a}{1-a}$, and the constant $c$ is given by $(100)$.

\section{Calculation of parameters in Table [}

We present the computation of $\theta_{0}, \theta_{1}, \theta_{2}$ in Table I. First, we restate the definition of $\theta_{r}(r=0, \ldots, q)$ ([24, Sec. 1.1], where $\mu$ is used instead of $\theta$ here). Given a multilinear polynomial $f$ in $z_{1}, \ldots, z_{n}$ of total degree $m$, one can construct a hypergraph $H=(\mathcal{V}(H), \mathcal{H}(H))$ as follows. The vertex set is $\mathcal{V}(H)=[n]$, and a hyperedge $h$ is a set $h \subset \mathcal{V}(H)$ of $|h| \leq m$ vertices. Mapping each monomial in $f$ to a hyperedge $h$ with weight $w_{h}$, we can express $f$ as

$$
f\left(z_{1}, \ldots, z_{n}\right)=\sum_{h \in \mathcal{H}(H)} w_{h} \prod_{v \in h} z_{v} .
$$

${ }^{9}$ Note that $\tilde{\mathrm{A}} \tilde{\mathrm{A}}^{T}$ and $\tilde{\mathrm{A}}^{T} \tilde{\mathrm{A}}$ have the same eigenvalues.
Definition 3 (Parameters $\theta_{r}, r=0, \ldots, m$, [24, Sec. 1.1], particularized to Gaussian random variables). Given a multilinear function $f$ of total degree $m$ and i.i.d. Gaussian random variables $Z_{1}, \ldots, Z_{n}$ with $Z_{1} \sim \mathcal{N}\left(0, \sigma^{2}\right)$. For $r=0, \ldots, m$, $\theta_{r}$ is defined as

$$
\theta_{r} \triangleq \max _{h_{0} \subset[n]:\left|h_{0}\right|=r}\left(\sum_{h \in \mathcal{H}(H): h_{0} \subset h} w_{h} \prod_{v \in h \backslash h_{0}} \mathbb{E}\left[\left|Z_{v}\right|\right]\right) .
$$

We present a simple example to illustrate Definition 3 Take $f\left(z_{1}, z_{2}, z_{3}\right)=z_{1} z_{2}+3 z_{2} z_{3}+z_{1} z_{2} z_{3}$, then $\mathcal{V}(H)=\{1,2,3\}$ and $\mathcal{H}(H)=\{\{1,2\},\{2,3\},\{1,2,3\}\}$. The correspoding weights are $1,3,1$, and $m=3$. It is easy to see that $\mathbb{E}\left[\left|Z_{i}\right|\right]=\frac{\sigma \sqrt{2}}{\sqrt{\pi}}$ for $Z_{i} \sim \mathcal{N}\left(0, \sigma^{2}\right)$. By Definition 3 for $f$ in this example, simple calculations yield $\theta_{1}=\frac{4 \sqrt{2} \sigma}{\sqrt{\pi}}+\frac{2 \sigma^{2}}{\pi}$.

Next, recall that the multilinear polynomial $f$, defined in 359, is of total degree $m=2$. By Definition 3 , to compute $\theta_{0}$, we take $h_{0}=\emptyset$ in (364):

$$
\begin{aligned}
\theta_{0} & =\sum_{h \in \mathcal{H}(H)} w_{h} \prod_{v \in h} \mathbb{E}\left[\left|Z_{v}\right|\right] \\
& =\sum_{j=1}^{n-1} \sum_{i=j+1}^{n} a^{i-1-j} \mathbb{E}\left[\left|Z_{j}\right|\right] \mathbb{E}\left[\left|Z_{i}\right|\right] \\
& =\frac{2 \sigma^{2}}{\pi} \sum_{j=1}^{n-1} \sum_{i=j+1}^{n} a^{i-1-j} \\
& =\frac{2 \sigma^{2}}{(1-a) \pi}\left(n-\frac{1-a^{n}}{1-a}\right),
\end{aligned}
$$

which implies

$$
\frac{2 \sigma^{2}}{(1-a) \pi}\left(n-\frac{1}{1-a}\right) \leq \theta_{0} \leq \frac{2 \sigma^{2} n}{(1-a) \pi} .
$$

To compute $\theta_{1}$, we have $h_{0} \subset[n]$ of size $\left|h_{0}\right|=1$ in (364). Equivalently,

$$
\begin{aligned}
\theta_{1} & =\max _{j \in[n]} \sum_{i=1}^{j-1} a^{j-i-1} \mathbb{E}\left[\left|Z_{i}\right|\right]+\sum_{i=j+1}^{n} a^{i-j-1} \mathbb{E}\left[\left|Z_{i}\right|\right] \\
& =\max _{j \in[n]} \frac{\sigma \sqrt{2}}{\sqrt{\pi}} \frac{2-a^{j-1}-a^{n-j}}{1-a} \\
& \leq \frac{2 \sqrt{2} \sigma}{\sqrt{\pi}(1-a)}
\end{aligned}
$$

As for $\theta_{2}$, we have $\theta_{2}=0$ since when $r=m=2$, we have $|h| \leq 2=\left|h_{0}\right|$, which implies that the sum in (364) is empty.

\section{REFERENCES}

[1] C. E. Shannon, "A mathematical theory of communication," The Bell System Technical Journal, vol. 27, no. 3, pp. 379-423, Jul. 1948.

[2] — "Coding theorems for a discrete source with a fidelity criterion," IRE Nat. Conv. Rec, vol. 4, no. 1, pp. 142-163, Mar. 1959.

[3] T. Goblick, "A coding theorem for time-discrete analog data sources," IEEE Transactions on Information Theory, vol. 15, no. 3, pp. 401-407, May 1969.

[4] R. Gray, "Information rates of autoregressive processes," IEEE Transactions on Information Theory, vol. 16, no. 4, pp. 412-421, Jul. 1970. 
[5] S. Jalali and T. Weissman, "New bounds on the rate-distortion function of a binary Markov source," in Proceedings of 2007 IEEE International Symposium on Information Theory, Nice, France, Jun. 2007, pp. 571 -575 .

[6] R. Gray, "Rate distortion functions for finite-state finite-alphabet Markov sources," IEEE Transactions on Information Theory, vol. 17, no. 2, pp 127 - 134, Mar. 1971.

[7] L. D. Davisson, "Rate-distortion theory and application," Proceedings of the IEEE, vol. 60, no. 7, pp. 800-808, Jul. 1972.

[8] A. Kolmogorov, "On the Shannon theory of information transmission in the case of continuous signals," IRE Transactions on Information Theory, vol. 2, no. 4, pp. 102-108, Dec. 1956.

[9] T. M. Cover and J. A. Thomas, Elements of information theory, 2nd ed. John Wiley \& Sons, Nov. 2012.

[10] T. Berger, "Rate-distortion theory," Encyclopedia of Telecommunications, 1971.

[11] Z. Zhang, E.-H. Yang, and V. K. Wei, "The redundancy of source coding with a fidelity criterion. I. Known statistics," IEEE Transactions on Information Theory, vol. 43, no. 1, pp. 71-91, Jan. 1997.

[12] E.-H. Yang and Z. Zhang, "On the redundancy of lossy source coding with abstract alphabets," IEEE Transactions on Information Theory, vol. 45, no. 4, pp. 1092-1110, May 1999.

[13] V. Kostina and S. Verdú, "Fixed-length lossy compression in the finite blocklength regime," IEEE Transactions on Information Theory, vol. 58, no. 6, pp. 3309-3338, Jun. 2012.

[14] A. Ingber and Y. Kochman, "The dispersion of lossy source coding," in Proceedings of 2011 Data Compression Conference (DCC), Snowbird, Utah, USA, Mar. 2011, pp. 53-62.

[15] V. Kostina and S. Verdú, "Lossy joint source-channel coding in the finite blocklength regime," IEEE Transactions on Information Theory, vol. 59, no. 5, pp. 2545-2575, May 2013.

[16] D. Wang, A. Ingber, and Y. Kochman, "The dispersion of joint sourcechannel coding," in Proceedings of 49th Annual Allerton Conference on Communication, Control, and Computing, Monticello, IL, USA, Sept. 2011, pp. 180-187.

[17] S. Watanabe, "Second-order region for Gray-Wyner network," IEEE Transactions on Information Theory, vol. 63, no. 2, pp. 1006-1018, Feb. 2017.

[18] V. Y. Tan and O. Kosut, "On the dispersions of three network information theory problems," IEEE Transactions on Information Theory, vol. 60, no. 2, pp. 881-903, Feb. 2014.

[19] I. Kontoyiannis, "Pointwise redundancy in lossy data compression and universal lossy data compression," IEEE Transactions on Information Theory, vol. 46, no. 1, pp. 136-152, Jan. 2000.

[20] A. A. Yushkevich, "On limit theorems connected with the concept of entropy of Markov chains," Uspekhi Matematicheskikh Nauk, vol. 8 , no. 5, pp. 177-180, 1953.

[21] I. Kontoyiannis and S. Verdú, "Optimal lossless data compression: Nonasymptotics and asymptotics," IEEE Transactions on Information Theory, vol. 60, no. 2, pp. 777-795, Feb. 2014.

[22] Y. Polyanskiy, H. V. Poor, and S. Verdú, "Dispersion of Gaussian channels," in Proceedings of 2009 IEEE International Symposium on Information Theory, Seoul, Korea, Jun. 2009, pp. 2204-2208.

[23] _ , "Dispersion of the Gilbert-Elliott channel," IEEE Transactions on Information Theory, vol. 57, no. 4, pp. 1829-1848, Apr. 2011.

[24] W. Schudy and M. Sviridenko, "Concentration and moment inequalities for polynomials of independent random variables," in Proceedings of 23rd Annual ACM-SIAM Symposium on Discrete Algorithms, Kyoto, Japan, Jan. 2012, pp. 437-446.

[25] T. Hashimoto and S. Arimoto, "On the rate-distortion function for the nonstationary Gaussian autoregressive process (Corresp.)," IEEE Transactions on Information Theory, vol. 26, no. 4, pp. 478-480, Jul. 1980.

[26] R. M. Gray and T. Hashimoto, "A note on rate-distortion functions for nonstationary Gaussian autoregressive processes," IEEE Transactions on Information Theory, vol. 54, no. 3, pp. 1319-1322, Feb. 2008.

[27] J. C. Kieffer, "Strong converses in source coding relative to a fidelity criterion," IEEE Transactions on Information Theory, vol. 37, no. 2, pp. 257-262, Mar. 1991.

[28] R. G. Gallager, Information theory and reliable communication. Springer, 1968, vol. 2.

[29] R. Blahut, "Computation of channel capacity and rate-distortion functions," IEEE Transactions on Information Theory, vol. 18, no. 4, pp. 460-473, Jul. 1972.

[30] I. Csiszár, "On an extremum problem of information theory," Studia Scientiarium Mathematicarum Hungarica, vol. 9, pp. 57-61, 1974.
[31] V. Kostina and E. Tuncel, "The rate-distortion function for successive refinement of abstract sources," in Proceedings of 2017 IEEE International Symposium on Information Theory, Aachen, Germany, Jun. 2017, pp. 1923-1927.

[32] A. Dembo and I. Kontoyiannis, "Source coding, large deviations, and approximate pattern matching," IEEE Transactions on Information Theory, vol. 48, no. 6, pp. 1590-1615, Jun. 2002.

[33] I. Dumer, M. S. Pinsker, and V. V. Prelov, "On coverings of ellipsoids in Euclidean spaces," IEEE Transactions on Information Theory, vol. 50 , no. 10, pp. 2348-2356, Oct. 2004.

[34] W. Feller, An introduction to probability theory and its applications. New York: Wiley, 1971, vol. 2.

[35] L. V. Ahlfors, Complex analysis: an introduction to the theory of analytic functions of one complex variable, 3rd ed. McGraw-Hill, 1979.

[36] R. Bhatia, Matrix analysis. Springer Science \& Business Media, 2013, vol. 169 .

[37] R. S. Varga, Matrix iterative analysis. Springer Science \& Business Media, 2009, vol. 27.

[38] S. Noschese, L. Pasquini, and L. Reichel, "Tridiagonal Toeplitz matrices: properties and novel applications," Numerical linear algebra with applications, vol. 20, no. 2, pp. 302-326, Mar. 2013. 